University of Louisville

ThinkIR: The University of Louisville's Institutional Repository

Electronic Theses and Dissertations

$5-2011$

\title{
Quantum and classical description of spin systems with application to coherent relaxation in a resonator.
}

Andrey Klots

University of Louisville

Follow this and additional works at: https://ir.library.louisville.edu/etd

\section{Recommended Citation}

Klots, Andrey, "Quantum and classical description of spin systems with application to coherent relaxation in a resonator." (2011). Electronic Theses and Dissertations. Paper 764.

https://doi.org/10.18297/etd/764

This Master's Thesis is brought to you for free and open access by ThinkIR: The University of Louisville's Institutional Repository. It has been accepted for inclusion in Electronic Theses and Dissertations by an authorized administrator of ThinkIR: The University of Louisville's Institutional Repository. This title appears here courtesy of the author, who has retained all other copyrights. For more information, please contact thinkir@louisville.edu. 


\title{
QUANTUM AND CLASSICAL DESCRIPTION OF SPIN SYSTEMS WITH APPLICATION TO COHERENT RELAXATION IN A RESONATOR
}

\author{
By \\ Andrey Klots \\ B.S., Perm State University, 2009 \\ A Thesis \\ Submitted to the Faculty of the \\ College of Arts and Sciences of the University of Louisville \\ In Partial Fulfillment of the Requirements \\ for the Degree of \\ Master of Science \\ Department of Physics and Astronomy \\ University of Louisville \\ Louisville, Kentucky
}

May 2011 


\title{
QUANTUM AND CLASSICAL DESCRIPTION OF SPIN SYSTEMS WITH APPLICATION TO COHERENT RELAXATION IN RESONATOR
}

\author{
By \\ Andrey Klots \\ B.S., Perm State University, 2009
}

Thesis Approved on

April 12, 2011

By the following thesis committee:

Victor Henner, Thesis Director

C. L. Davis, Co-advisor 


\section{ABSTRACT \\ QUANTUM AND CLASSICAL DESCRIPTION OF SPIN SYSTEMS WITH APPLICATION TO COHERENT RELAXATION IN A RESONATOR}

Andrey Klots

April 1, 2011

This paper describes effects of the interaction between the spin system and the resonator in magnetic resonance. In NMR the resonator circuit can provide feedback to the system of spins, which can lead to unusual effects, such as increasing dipole interactions and multiple flips [3]. 


\section{TABLE OF CONTENTS}

PAGE

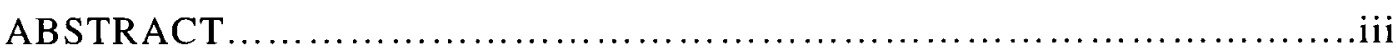

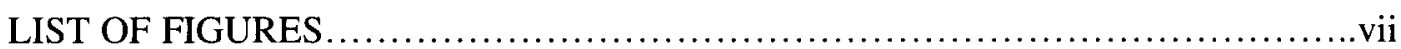

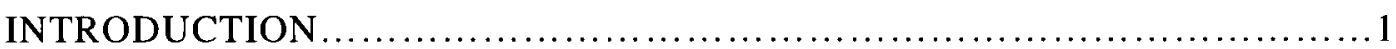

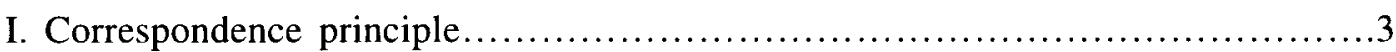

Comparison of quantum and classical approaches................................. 3

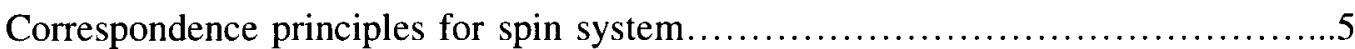

1. Correspondence principle for non-interacting

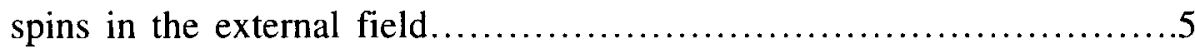

2. Correspondence principle for interacting magnetic moments............6

3. Correspondence principle for other observables........................ 9

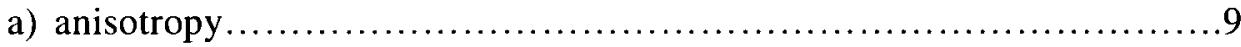

b) Correspondence principle for the magnitude of the total spin in the presence of DD-interactions.......................................12

c) Correspondence principle for the length of the total spin in the case of different non-interacting particles in an external field...................17

II. Quantum system of equations. Splitting the Hamiltonian into

Secular and Non-Secular terms. Correspondence principle for the

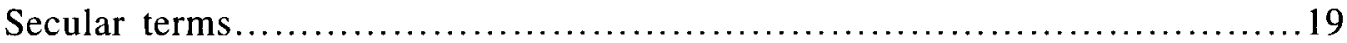

1. Commutation of the Z-component of spins with the secular Hamiltonian......................................

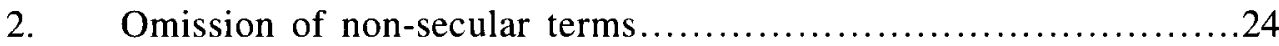

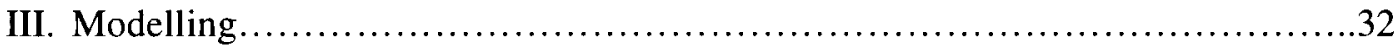

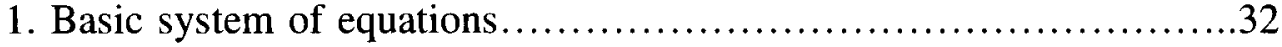

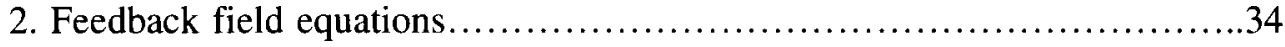

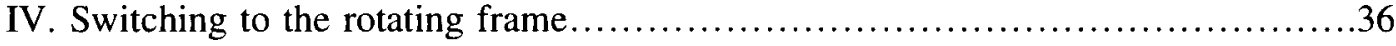


1. Equations for the induced field of the coil in the

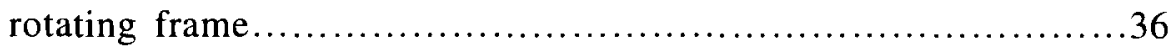

2. Equations for spins in the rotating frame.........................39

$V$. Analyzing equations from the point

of view of the control theory. Interaction of a Spin

System with the Resonator and using this interaction for control of

Spin Polarization.................................................. 46

1. Behavior of a spin system inside a resonator.......................46

2. Resonator as a control system with feedback........................50

3. Generalization of the control theory approach......................56

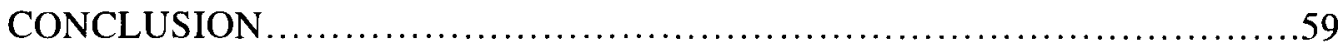

REFERENCES ..................................................6 60

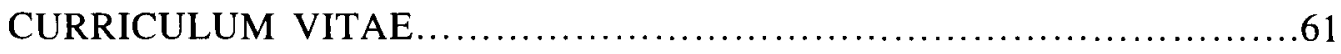




\section{LIST OF FIGURES}

PAGE

1. Spin system in passive resonator and external magnetic field $\ldots \ldots \ldots \ldots \ldots \ldots \ldots \ldots$

1.1 Diagram, describing algorithm of checking of validity of the correspondence principle. ............................... 3

1.3.b.1 Time evolution of components and length of the normalized total spin in presence of dipole interaction................15

1.3.c.1 Time evolution of components and length of the normalized total spin in case of different gyrommagnetic ratios.............. 17

2.1.1 Time evolution of the Z-component of the total spin in case of secular dipolar interactions...............................23

2.2.1 System of spins, arranged in the "magic angle" direction......................26

2.2.2 Time evolution of components of the total spin in cases of different alignments, types and strengths of dipolar interaction..............30

4.1.1 Decomposition of the field of resonator into $\mathrm{CW}$ and $\mathrm{CCW}$ components

4.2.1 Flowchart of the algorithm of simulation of spin dynamics in presence of the resonator in the rotating frame

4.2.2 Effects of direct dipole interaction and interaction via resonator....

5.1.1 screenshot, demonstrating relaxation of a spin system via resistance of the resonator.

5.1.2 Screenshot, demonstrating relaxation of a spin system via resistance of the resonator (initial polarization is -0.5 )

5.1.3 Screenshot, demonstrating relaxation of a spin system via resistance of the resonator ( initial polarization is +0.5 )

5.1.4 effect of polarization of a spin sample in case of negative $\beta$

5.2.1 Control-theory diagram, describing interaction between our spin system and the resonator................................50

5.2.2 Control of amplitude of X-component of the spin.........................53

5.2.3 Screenshot with the magnetic field chart, zoomed time wise...................54

5.2.4 Screenshot with the magnetic field chart, zoomed time wise (at a later time moment).

5.2.5 Control of amplitude of X-component of the spin (bigger control signal). 
5.2.6 Control of amplitude of X-component of the spin

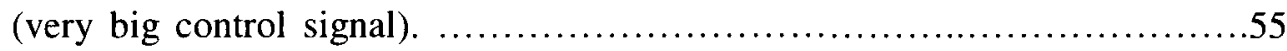

5.3.1 General control-theory diagram between two subsystems....................56

5.3.2 Detailed control diagram of interaction between two systems ..................57

5.3.3 Re-arranged detailed control diagram of interaction between

two systems........................................................... 58 


\section{INTRODUCTION}

Solving quantum-mechanical problems is often very difficult because they are equivalent to problems of linear algebra in a huge (often infinite) number of dimensions. However, in the case of macroscopic systems, operators are reduced to functions, for which equations become much simpler. This transition can be performed with the help of the correspondence principle, which originates from the fact that at macroscopic scales effects such as non-commutativity of operators can be neglected and hermitian operators, therefore, behave just as real numbers.

Interestingly enough, sometimes the correspondence principle can be used even for microscopic systems. One of the brightest examples of this effect is the Ehrenfest theorem, which shows that for a quantum-mechanical particle the equation for an operator of linear momentum is $\hat{\vec{p}}=-\nabla \hat{U}$. This equation formally corresponds to the classical second Newton's law combined with the relationship between a potential energy and force: $\vec{F}=-\nabla U$. This important theorem highlights the relationship between the quantum and classical worlds.

The natural question which arises, when we look at this elegant equation is: can we get similar "microscopic" correspondence principles for other values? Can this correspondence principle take place for a pure quantum value, such as spin? As the reader will be able to see in this paper, such a correspondence really exists! Of course, it is well known that, for example, in the theory of magnetic resonance, for spins in a classical external magnetic field, quantum and classical approaches give similar results. But will the results coincide if we include such effects as dipole interactions or anisotropy in the problem? Does the validity of the correspondence principle for some set of observables lead to correspondence for various functions of those observables? We will try to answer these and other question in this paper.

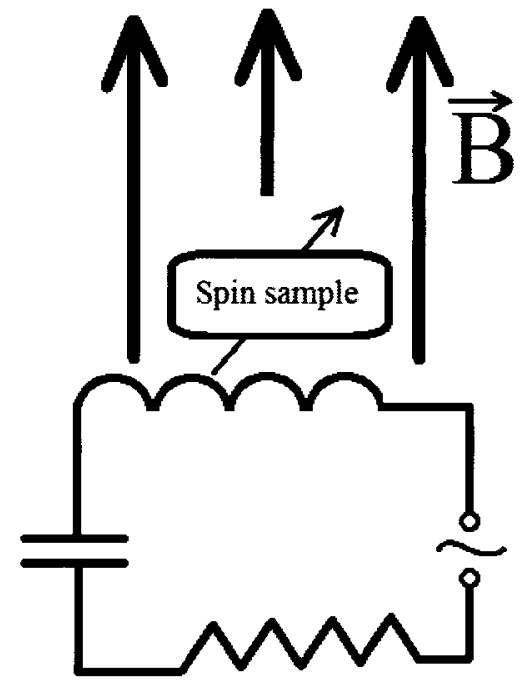

Figure 1. Spin system in passive resonator and external magnetic field

Purely mathematical-physical problems set up above are meaningless, if they do not contribute to solution of practical problems. Thus, the second part of this paper will be devoted to solving the problem of the interaction between a spin-system and a resonator. In magnetic resonance the 
resonator is used to create a high-frequency horizontal magnetic field, which flips the spin of a sample, which is placed in magnetic field $\vec{B}$ (see fig. 1 ).

Since a resonator is nothing but an LRC-circuit, due to the effect of electro-magnetic induction, it can provide a feedback to the spin system. Since the resonator frequency is usually adjusted to the Zeeman frequency of spins in the sample, the LRC-circuit will be in resonance with the oscillating of spins in a sample. Each spin individually contributes to the total magnetic moment of the sample. The evolution of the magnetic moment induces an electromotive force in the LRC circuit. This electromotive force creates a feedback magnetic field of the coil, which acts on each spin of the system individually. Thus, the resonator provides not only feedback for the spin system as a whole, but also creates an additional interaction between spins. The second part of this paper will describe some of the interesting effects which occur due to the resonator. In order to do this we suggest the model, based on the correspondence principle, described in the beginning.

The current document consists of five major parts.

In the first part we will analyze the possibility of switching from quantum Heisenberg equations to classical equations. This will allow us to simplify the process of analyzing and modeling dynamics of the system.

The second part describes an idea, probably familiar to the reader, of splitting the Hamiltonian of dipolar interaction into secular and non-secular terms. Also this part shows how the model designed on the basis of principles, derived in part I, coincides with prediction of the quantum approach.

After the framework is set up, part three describes the model, based on numerical solution of the system of equations of spin dynamics along with equations of the resonator.

The fourth part is devoted to switching to the rotating frame - a basic technique, used to get rid of big terms, which appear in the dynamical equations due to the Zeeman field. However, if we want to take interaction with the resonator into account, switching to the rotating frame becomes more complicated.

In the fifth part we will analytically (mathematical framework of the control theory) describe some of the effects, observed in our numerical model and predict some other interesting effects. 


\section{CORRESPONDENCE PRINCIPLE}

\section{Comparison of Quantum and Classical Approaches}

In this part we will analyze reasons, due to which we can (or cannot) to switch from Schrodinger's or Heisenberg's equations to classical ones. We will figure out the correspondence between Heisenberg's equations and the classical equations of spin dynamics. This will allow us to determine the range of situations, where we can switch from Heisenberg's equation to classical ones. The following diagram illustrates the strategy we are going to use:

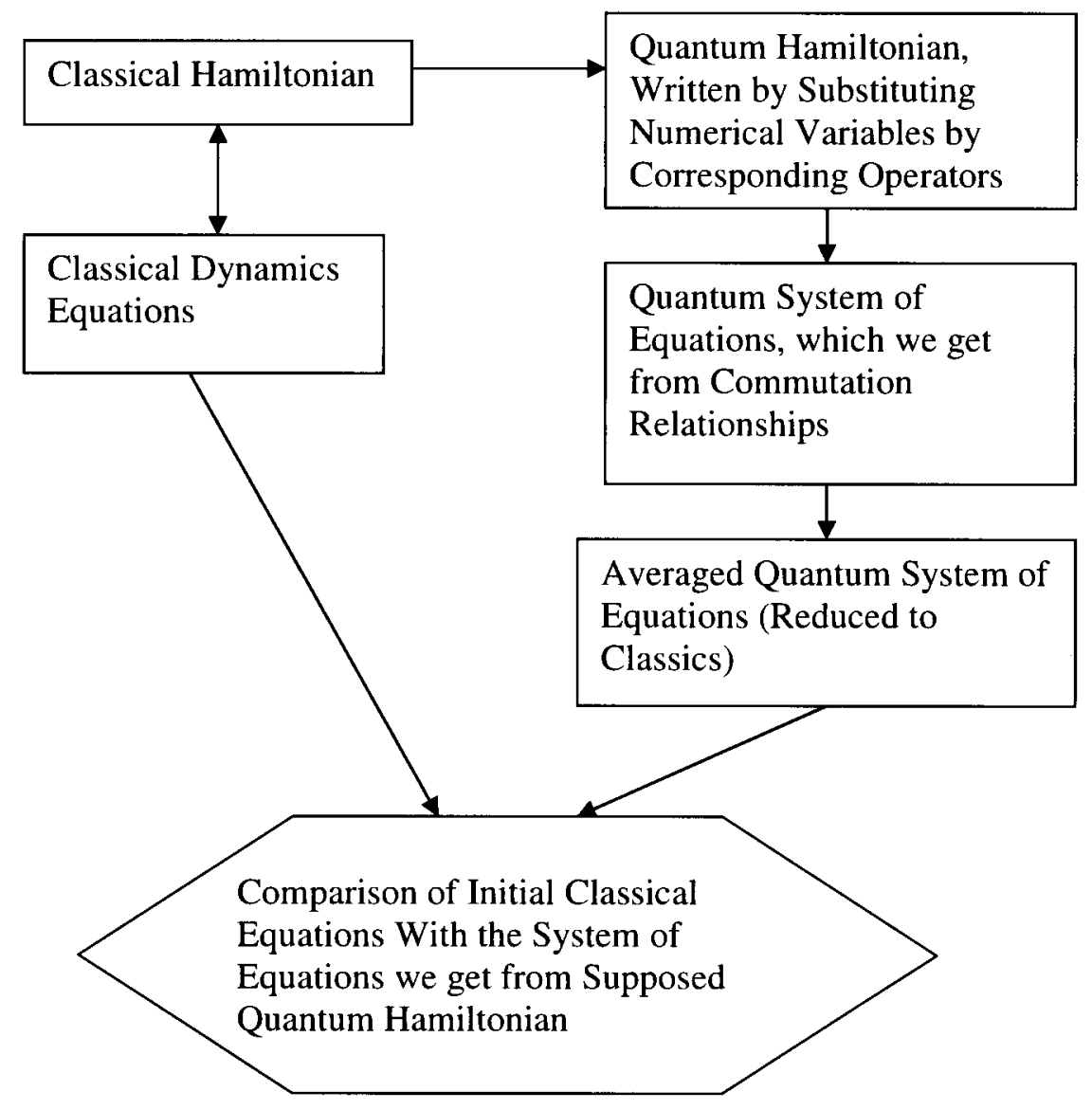

Figure 1.1 Diagram, describing algorithm of checking of validity of the correspondence principle. 
After that we will consider the problem of big terms in the Hamiltonian and switching to the rotating frame as the solution of this problem. Finally, we will get equations for spin dynamics in the rotating frame taking into account the feedback due to the presence of a resonator and create the algorithm based on these equations.

One can write the quantum equation as

$$
i \frac{\partial}{\partial t}|\psi\rangle=\left\{-\vec{B}\left(\gamma_{s} \hbar\right) \hat{\vec{S}}+\hat{\mathscr{H}}_{d d}+\hat{\mathscr{H}}_{\text {anisor }}\right\}|\psi\rangle
$$

Here $\gamma_{s}$ is the gyromagnetic ratio, relating spin of the particle to its magnetic moment. The first term in the right-hand side corresponds to the external magnetic field, second - to the dipole-dipole interaction, and the third to anisotropy. If we write this equation for the system of $\mathrm{N}$ spins of magnitude $\mathrm{S},|\psi\rangle$ will have $(2 s+1)^{N}$ components. Having such a fast growth of complexity of a problem with growth of $\mathrm{N}$, the exact solution for a big number of particles becomes practically impossible.

One solution is to use the mean field approximation. In this case the state vector of the system of particles will be nothing but a direct product of individual state vectors of all the particles of the system, which leads to a linear growth of complexity of the problem with growth of $\mathrm{N}$.

On the other hand, due to use of the mean-field approximation, such purely quantum effects as entanglement do not take place, consequently, if we prove the correspondence principle between quantum and classical descriptions of spin dynamics, we will be able to switch from quantum equations to classical ones:

$$
\left\{\begin{array}{c}
\dot{S}_{i}^{x}=-\omega_{0} S_{i}^{y}+\frac{\omega_{D}}{S} S_{i}^{z} S_{i}^{y}+D_{x} \\
\dot{S}_{i}^{y}=\omega_{0} S_{i}^{x}+\gamma_{s}\left(B_{1}+H\right) S_{i}^{z}-\frac{\omega_{D}}{S} S_{i}^{z} S_{i}^{x}+D_{y} \\
\dot{S}_{i}^{z}=-\gamma_{s}\left(B_{1}+H\right) S_{i}^{y}+D_{z} \\
\frac{d^{2} H}{d t^{2}}+2 \gamma \frac{d H}{d t}+\omega^{2} H=-4 \pi \frac{d^{2} m_{x}}{d t^{2}}
\end{array}\right.
$$

(derivation of these equations will be shown in Part III)

Here $S_{i}^{x, y, z}$ are classical spins, $\omega_{0}$ - is the Zeeman frequency, $\frac{\omega_{D}}{S}$ characterizes the anisotropy, $\left(B_{1}+H\right)$ - fields, created by the coil (external fields and a field induced 
due to the resonator respectively), $D_{x, y, z}$ - describe contributions of dipole-dipole interactions $D_{x, y, z}=\left\langle\frac{-i}{\hbar}\left[\hat{S}_{j}^{x, y, z}, \hat{H}_{d d}\right]\right\rangle$. The last equation describes feedback through the self-inductance of the resonator.

\section{Correspondence Principles for Spin System}

Let us begin with the obvious equivalence between quantum and classical descriptions of the time evolution of a spin (magnetic moment) in an external magnetic field. It is clear that the Heisenberg equation for the magnetic moment operator $\hat{\vec{\mu}}$ in classical external field $\vec{H}$ (Hamiltonian $\hat{\boldsymbol{H}}=-\hat{\vec{\mu}} \vec{H}$ )

$$
\dot{\hat{\vec{\mu}}}=\frac{-i}{\hbar}[\hat{\vec{\mu}}, \hat{H}],
$$

is equivalent to the classical rotation equation

$$
\frac{d \vec{\mu}}{d t}=\gamma_{s} \vec{\mu} \times \vec{H}
$$

The same correspondence takes place for the system of many non-interacting magnetic moments. This correspondence looks trivial, but does this correspondence take place in the case of interacting particles, accounting for other factors, such as interaction with an external feedback field (if the system is inside the resonator), and in the presence of anisotropy? In fact we are going to derive an analog of the Ehrenfest's theorem for the system of interacting magnetic moments. The validity of such a correspondence allows us to justify modeling the dynamics of spin systems with the help of classical equations.

\section{Correspondence Principle for Non-Interacting Spins in the External Field}

Let us denote the external field as $\vec{H}_{0}$, then $\vec{H}_{0}=\sum_{\alpha} H^{\alpha} \vec{e}^{\alpha}$, where $\vec{e}^{\alpha}$ are unitary vectors in $\mathrm{x}, \mathrm{y}, \mathrm{z}$ directions. Starting from here we will be using Latin letters for particle numbers and Greek letters for spatial dimensions. Also, summation over repeating Greek indexes is implied. Besides, we will use dimensionless spins. The external field can depend on coordinates and time.

is

In the quantum description, based on Heisenberg's equations, the Hamiltonian

$$
\hat{\mathcal{H}}_{0}=-\sum_{j} \hat{\vec{\mu}}_{j} \vec{H}_{0}=-\hbar \sum_{j} \gamma_{j} H^{\alpha} \hat{S}_{j}^{\alpha}
$$

here $\hat{\vec{\mu}}_{j}=\gamma_{j} \hbar \hat{\vec{S}}_{j}, \gamma_{j}$ - gyromagnetic ratio of $j^{\text {th }}$ particle $\left(-g_{s}|e| / 2 m c\right.$ for electron; for electron spin $g_{s} \approx 2$ ). 
The dynamical equations are

$$
\dot{\hat{S}}_{l}^{\gamma}=\frac{-i}{\hbar}\left[\hat{S}_{l}^{\gamma}, \hat{\mathcal{H}}_{0}\right]=\frac{-i}{\hbar}\left[\hat{S}_{l}^{\gamma},-\hbar \sum_{j} \gamma_{j} H^{\alpha} \hat{S}_{j}^{\alpha}\right]=i \sum_{j} \gamma_{j} H^{\alpha}\left[\hat{S}_{l}^{\gamma}, \hat{S}_{j}^{\alpha}\right] \text {. }
$$

With $\left[\hat{S}_{l}^{\gamma}, \hat{S}_{j}^{\alpha}\right]=i \delta_{l j} e_{\beta \gamma \alpha} \hat{S}_{l}^{\beta}$ we get

$\dot{\hat{S}}_{l}^{\gamma}=-\gamma_{l} e_{\beta \gamma \alpha} H^{\alpha} \hat{S}_{l}^{\beta}=\gamma_{l} e_{\gamma \beta \alpha} \hat{S}_{l}^{\beta} H^{\alpha}$, or in vector form:

$\dot{\overrightarrow{\hat{S}}}_{l}=\gamma_{l} \hat{\vec{S}}_{l} \times \vec{H}_{0}$.

In the classical description

$\dot{\vec{\mu}}_{l}=\gamma_{l} \vec{\mu}_{l} \times \vec{H}_{0}$ and $\dot{\vec{S}}_{l}=\gamma_{l} \vec{S}_{l} \times \vec{H}_{0}$,

where $\vec{\mu}_{l}$ and $\vec{S}_{l}$ are classical vectors.

Thus, we see correspondence between classical and quantum equations.

In the case of equal for all spins $\gamma_{I} \equiv \gamma_{s}$, summing up the equations (either quantum or classical), we have

$$
\frac{d}{d t} \sum_{l} \hat{\vec{S}}=\sum_{l} \gamma_{l} \hat{\vec{S}}_{l} \times \vec{H}=\gamma_{s} \sum_{l} \hat{\vec{S}}_{l} \times \vec{H}-\text { this equation for total spin looks exactly }
$$

like for an individual spin.

Our external field is the sum of the Zeeman field and resonator's field ( $\vec{H}_{0}=\vec{B}+\vec{H}$ ). If in our problem $\vec{H}$ is a classical field of the feedback due to resonator and is similar to all the spins, the correspondence principle will remain valid in the presence of the resonator.

\section{Correspondence Principle for Interacting Magnetic Moments}

The dipole energy of a system of classical magnetic moments

$$
E_{d d}=\frac{1}{2} \sum_{l k}\left(\frac{\vec{\mu}_{l} \vec{\mu}_{k}}{r_{l k}^{3}}-\frac{3}{r_{l k}^{5}}\left(\vec{\mu}_{l} \vec{r}_{l k}\right)\left(\vec{\mu}_{k} \vec{r}_{l k}\right)\right)
$$

Can be also written as

$$
E_{d d}=\frac{1}{2} \sum_{k l} D_{l k}^{\alpha \beta} \mu_{l}^{\alpha} \mu_{k}^{\beta}
$$

(again, summation over Greek indexes is implied), where

$$
D_{l k}^{\alpha \beta}=\frac{1}{r_{l k}^{3}} \delta_{\alpha \beta}-\frac{3}{r_{l k}^{5}} r_{l k}^{\alpha} r_{l k}^{\beta}
$$

The tensor $D_{l k}^{\alpha \beta}$ is symmetric over lower and upper indexes, Greek indexes correspond to vectors, Latin - to particle numbers. 
The energy of the $l$-th magnetic moment in the field of others: $E_{(d d) l}=-\vec{H}_{l} \vec{\mu}_{l}$, or

$$
E_{(d d) l}=-H_{l}^{\alpha} \mu_{l}^{\alpha},
$$

Then, the magnetic (dipole) field at the place of $l$-th magnetic moment is given by

$$
H_{l}^{\alpha}=-\partial E_{d d} / \partial \mu_{l}^{\alpha}=-\sum_{k \neq l} D_{l k}^{\alpha \beta} \mu_{k}^{\beta}
$$

The dynamics of a vector $\vec{\mu}_{l}$ in the dipole magnetic field is described by equation

$$
\dot{\vec{\mu}}_{l}=\gamma_{i}\left[\vec{\mu}_{l} \times \vec{H}_{l}\right]
$$

From here

$$
\dot{\mu}_{l}^{\gamma}=\gamma_{l} e_{\alpha \sigma \gamma} H_{l}^{\alpha} \mu_{l}^{\sigma}=\gamma_{l} \sum_{k} e_{\gamma \alpha \sigma} D_{l k}^{\alpha \beta} \mu_{k}^{\beta} \mu_{l}^{\sigma}
$$

Now, let us consider a quantum description. Operators of magnetic moment and spin are related as $\left(\gamma_{s}=e / 2 m c<0\right.$ for electrons, $\left.\mu=-2 \mu_{B}, \mu=\hbar \gamma_{s}\right)$

$$
\hat{\vec{\mu}}_{l}=\gamma_{l} \hbar \hat{\vec{S}}_{l} \text {. }
$$

The contribution of dipolar interactions into $\hat{\overrightarrow{\vec{S}}}_{l}$ (we write Hamiltonian $\hat{\mathscr{H}}_{d d}$ in terms of spin operators).

$$
\dot{\hat{S}}_{l}^{\gamma}=\frac{-i}{\hbar}\left[\hat{S}_{l}^{\gamma}, \hat{\mathcal{H}}_{d d}\right]=\frac{-i \hbar^{2}}{\hbar}\left[\hat{S}_{l}^{\gamma}, \frac{1}{2} \sum_{j k} \gamma_{j} \gamma_{k} D_{j k}^{\alpha \beta} \hat{S}_{j}^{\alpha} \hat{S}_{k}^{\beta}\right] .
$$

We can calculate this commutator, considering $j \neq k$ and $[a, b c]=[a, b] c+b[a, c]$ :

$$
\left[\hat{S}_{l}^{\gamma}, \hat{S}_{j}^{\alpha} \hat{S}_{k}^{\beta}\right]=\left[\hat{S}_{l}^{\gamma}, \hat{S}_{j}^{\alpha}\right] \hat{S}_{k}^{\beta}+\hat{S}_{j}^{\alpha}\left[\hat{S}_{l}^{\gamma}, \hat{S}_{k}^{\beta}\right]=i e_{\gamma \alpha \sigma} \hat{S}_{l}^{\sigma} \delta_{l j} \hat{S}_{k}^{\beta}+i e_{\gamma \beta \sigma} \hat{S}_{j}^{\alpha} \hat{S}_{l}^{\sigma} \delta_{l k} .
$$

Then

$$
\begin{aligned}
& \dot{\hat{S}}_{l}^{\gamma}=\hbar \frac{1}{2} \sum_{j k} \gamma_{j} \gamma_{k}\left(e_{\gamma \alpha \sigma} \delta_{l j} D_{j k}^{\alpha \beta} \hat{S}_{l}^{\sigma} \hat{S}_{k}^{\beta}+e_{\gamma \beta \sigma} \delta_{l k} D_{j k}^{\alpha \beta} \hat{S}_{j}^{\alpha} \hat{S}_{l}^{\sigma}\right)= \\
& =\hbar \frac{1}{2}\left(\sum_{k} \gamma_{l} \gamma_{k} e_{\gamma \alpha \sigma} D_{l k}^{\alpha \beta} \hat{S}_{l}^{\sigma} \hat{S}_{k}^{\beta}+\sum_{j} \gamma_{j} \gamma_{l} e_{\gamma \beta \sigma} D_{j l}^{\alpha \beta} \hat{S}_{j}^{\alpha} \hat{S}_{l}^{\sigma}\right)
\end{aligned}
$$

Since $D_{l k}^{\alpha \beta}$ is symmetric over lower and upper indexes, in the second term we can substitute $\alpha$ to $\beta$ and change the summation index $j$ to $k$, then

$$
\dot{\hat{S}}_{l}^{\gamma}=\hbar \gamma_{l} \frac{1}{2} \sum_{k} \gamma_{k} e_{\gamma \alpha \sigma} D_{l k}^{\alpha \beta}\left(\hat{S}_{l}^{\sigma} \hat{S}_{k}^{\beta}+\hat{S}_{k}^{\beta} \hat{S}_{l}^{\sigma}\right)
$$

And since $l \neq k$, 
$\dot{\hat{S}}_{l}^{\gamma}=\gamma_{l} \sum_{k} e_{\gamma \alpha \sigma} D_{l k}^{\alpha \beta}\left(\gamma_{k} \hbar \hat{S}_{k}^{\beta}\right) \hat{S}_{l}^{\sigma}$

For operators $\hat{\vec{\mu}}_{l}=\left(\gamma_{s} \hbar\right) \hat{\vec{S}}_{l}$ equation (1.2.10) results in $\dot{\hat{\mu}}_{l}^{\gamma}=\gamma_{1} \sum_{k} e_{\gamma \alpha \sigma} D_{l k}^{\alpha \beta} \hat{\mu}_{k}^{\beta} \hat{\mu}_{l}^{\sigma}$.

Equations (1.2.7) and (1.2.11) demonstrate the fairness of the correspondence principle for magnetic dipole interactions:

(a) for classical vectors $\vec{S}_{l}$ and $\dot{\vec{\mu}}_{l}$ :

$\dot{\vec{S}}_{l}=\gamma_{l} \vec{S}_{l} \times \vec{H}_{l}$ and $\dot{\vec{\mu}}_{l}=\gamma_{l} \vec{\mu}_{l} \times \vec{H}_{l}$,

where $\vec{H}_{l}$ - is a dipole part of magnetic field, defined by equation (1.2.5);

(b) for operators

$\dot{\hat{\vec{S}}}_{l}=\gamma_{l} \hat{\vec{S}}_{l} \times \hat{\vec{H}}_{l}$ and $\dot{\overrightarrow{\hat{\mu}}}_{l}=\gamma_{l} \hat{\vec{\mu}}_{l} \times \hat{\vec{H}}_{l}$,

where operator $\hat{\vec{H}}_{l}$ of dipole magnetic field is defined as

$$
\hat{H}_{l}^{\alpha}=-\sum_{k} D_{l k}^{\alpha \beta} \hat{\mu}_{k}^{\beta}
$$

Thus, we have proven equivalence of the two approaches for interacting magnetic moments: approach based on Heisenberg's equations, in which time evolution of the operator is defined by commuting the Hamiltonian with the corresponding operator, and the classical approach in which the magnetic moment $\vec{\mu}_{l}$ precesses in a magnetic field according to equation (1.2.12).

This result is based on the symmetry of tensor $D_{l k}^{\alpha \beta}$ over upper and lower indexes. With any particular values for $D_{l k}^{\alpha \beta}$, preserving this symmetry, e.g., if we split the Hamiltonian into secular and non-secular terms, the result will not change, the correspondence principle will take place for each term. Also, the correspondence principle is legitimate for any subset of set of particles of the entire spin system.

(1.2.10) and (1.2.13) - are operator equations, the correspondence principle allows, for instance, for computing modeling purposes, to switch to classical spins and magnetic moments. Further (for example, in part II) we will see that this approach leads us to correct results.

The quantum and classical approaches are completely equivalent for transitions between the states with greatest values of $|m|$. In this paper we will restrict ourselves with this often appeared in applications case.

Thus, the correspondence principle is valid for $\hat{S}_{j}^{\alpha}$ in external classical fields and for dipole interactions. This principle is not trivial (we haven't seen a corresponding discussion in the literature for the case including interacting magnetic moments) and as we mentioned is useful for studying spin systems. Let us check, if correspondence will remain for other operators, which are functions of $\hat{S}_{j}^{\alpha}$. These 
operators are, for example, anisotropy Hamiltonian $H_{\text {anis }}^{\text {: }}$ and the magnitude of the total spin $\left(\sum_{j} \hat{\vec{S}}_{j}\right)^{2}$.

\section{Correspondence Principle for Other Observables}

\section{a) Anisotropy}

Taking account of anisotropy is important for such cases as nanomolecules or ferromagnetic nanoparticles. Let us consider the correspondence of classical and quantum descriptions for the anisotropy Hamiltonian, typically written in the form (uniaxial anisotropy):

$$
\hat{H}_{\text {unis }}=-D \sum_{j} \hat{S}_{j}^{z} \hat{S}_{j}^{z} . \quad D>0 .
$$

In this case (in the classical situation) $E_{\text {anis }}=-D\left(\gamma_{s} \hbar\right)^{-2} \sum_{j} \mu_{j}^{z} \mu_{j}^{z}$ and the anisotropy field is aligned along z-direction:

$$
H_{u n i s}^{:}=-\partial E_{a n i s} / \partial \mu_{i}^{z}=\frac{2 D}{\left(\gamma_{s} \hbar\right)^{2}} \mu_{i}^{z}=\frac{2 D}{\left(\gamma_{s} \hbar\right)} S_{l}^{z} \text {. }
$$

Then

$$
\begin{aligned}
& \dot{\vec{S}}_{l}=\gamma_{s} \vec{S}_{l} \times \vec{H}_{l(a n i s)}=\frac{2 D}{\hbar} \vec{S}_{l} \times \vec{k} S_{l}^{z} \text { and } \\
& \dot{S}_{l}^{x}=\frac{2 D}{\hbar} S_{l}^{y} S_{l}^{z}, \quad \dot{S}_{l}^{y}=-\frac{2 D}{\hbar} S_{l}^{x} S_{l}^{z}, \quad \dot{S}_{l}^{z}=0
\end{aligned}
$$

In the quantum approach:

$$
\dot{\hat{S}}_{l}^{\gamma}=\frac{-i}{\hbar}\left[\hat{S}_{l}^{\gamma}, \hat{\mathscr{H}}_{\text {anis }}\right]
$$

If we use $[a, b c]=[a, b] c+b[a, c]$, and considering $\left.\hat{\mathscr{H}}_{\text {anis }}=-D \gamma_{s}^{-1} \sum_{j} \hat{S}_{j}^{z} \hat{S}_{j}^{z}\right)$

we get

$$
\left[\hat{S}_{l}^{x}, \sum_{j} \hat{S}_{j}^{z} \hat{S}_{j}^{z}\right]=\left[\hat{S}_{l}^{x}, \hat{S}_{l}^{z} \hat{S}_{l}^{z}\right]=-i \hbar\left(\hat{S}_{l}^{y} \hat{S}_{l}^{z}+\hat{S}_{l}^{z} \hat{S}_{l}^{y}\right) .
$$

Then $\hat{\dot{S}}_{l}^{x}=\frac{-i}{\hbar} i \hbar(-D) \gamma_{s}^{-1}\left(\hat{S}_{l}^{y} \hat{S}_{l}^{z}+\hat{S}_{l}^{z} \hat{S}_{l}^{y}\right)=-D \frac{1}{\gamma_{s} \hbar}\left(\hat{S}_{l}^{y} \hat{S}_{l}^{z}+\hat{S}_{l}^{z} \hat{S}_{l}^{y}\right)$

(1.3.a.4), $\dot{\hat{S}}_{l}^{y}=\frac{1}{\gamma_{s} \hbar} D\left(\hat{S}_{l}^{x} \hat{S}_{l}^{z}+\hat{S}_{l}^{z} \hat{S}_{l}^{x}\right)$ - which formally corresponds to the classical 
result $\left(\dot{S}_{i}^{y}=-\frac{2 D}{\hbar} S_{l}^{x} S_{l}^{z}=-\frac{D}{\hbar}\left(S_{l}^{x} S_{l}^{z}+S_{l}^{z} S_{l}^{x}\right)\right.$ ), though it is not a real correspondence.

This kind of result we shall call "formal correspondence" - that means that classical equation can be formally brought to the same form as the quantum one, since unlike the quantum situation, classically we are able to change the order of factors. In other words, in case of the formal correspondence there is an asymmetry between the quantum and classical equations. Classical equations can always be brought to the form of the quantum ones, but quantum equations cannot always be brought to the form of the classical ones. Formal correspondence indicates similarity of quantum and classical equations, but cannot be used for justification for modelling microscopic dynamics of corresponding value using classical equations. However, in classical limit (where $[\hat{A}, \hat{B}][\hbar \approx 0$ and therefore, $\hat{A} \hat{B} \approx \hat{B} \hat{A}$ and $\{\hat{A}, \hat{B}\} \approx 2 \hat{A} \hat{B}$ ) we still arrive at macroscopic correspondence. Thus, the legitimacy of the classical approximation is defined by the error we get by substituting noncommutating operators by commutating numbers, which can be defined from following consideration: if we change the order of operators $\left(\hat{S}^{\alpha} \hat{S}^{\beta}=\hat{S}^{\beta} \hat{S}^{\alpha}+i \hbar e_{\alpha \beta \gamma} \hat{S}^{\gamma}\right)$, we'll get the difference of the magnitude of $\hbar \hat{S}^{\gamma}$. Then, considering the fact that $\left\langle\hat{S}^{\alpha}\right\rangle \square \hbar S$ (S is the unitless spin length), the relative difference will be $\frac{\left\langle\hbar \hat{S}^{\gamma}\right\rangle}{\left\langle\hat{S}^{\alpha} \hat{S}^{\beta}\right\rangle} \square \frac{\hbar\left\langle\hat{S}^{\gamma}\right\rangle}{\left\langle\hat{S}^{\alpha}\right\rangle\left\langle\hat{S}^{\beta}\right\rangle} \square \frac{\hbar(\hbar S)}{(\hbar S)(\hbar S)}=\frac{1}{S}$.

For instance, if we are dealing with nanomolecules with relatively short spins ( $S \leq 10$ ), using a classical approximation will give us a significant error. If we are dealing with big spins (for example, for nanoferromagnets $S \square 10^{4}$ ) the classical approximation gives good precision.

For $S=1 / 2$ in both cases we get zero: all the anticommutators $(\gamma=1,2,3)$ are equal to zero. In this case parameter $D$ should be defined as zero for $S=1 / 2$ (i.e. for $S=1 / 2$ anisotropy, defined in such a manner does not contribute).

Thus, in the general case, we don't get a correspondence principle for anisotropy.

Nevertheless, there are several other ways to introduce anisotropy:

$$
\begin{aligned}
& \hat{H}_{\text {anis(2) }}=D\left(\hat{S}^{z}\right)^{2}+D_{1}\left(\hat{S}^{y}\right)^{2}+D_{4}\left[\left(\hat{S}^{x}\right)^{2}\left(\hat{S}^{y}\right)^{2}+\left(\hat{S}^{y}\right)^{2}\left(\hat{S}^{z}\right)^{2}+\left(\hat{S}^{z}\right)^{2}\left(\hat{S}^{x}\right)^{2}\right], \\
& \hat{H}_{\text {anis }(3)}=D^{\prime}\left(\hat{S}^{z}\right)^{4}
\end{aligned}
$$

For terms of the kind $D\left(\hat{S}^{\beta}\right)^{2},(\mathcal{Z})$ (for this paragraph there is no automatic summation over repeating Greek indexes) In the quantum case we will have: 


$$
\begin{aligned}
& \dot{\hat{S}}^{\alpha}=-\frac{i}{\hbar} D\left[\hat{S}^{\alpha},\left(\hat{S}^{\beta}\right)^{2}\right]=-i \frac{D}{\hbar}\left(\left[\hat{S}^{\alpha}, \hat{S}^{\beta}\right] \hat{S}^{\beta}+\hat{S}^{\beta}\left[\hat{S}^{\alpha}, \hat{S}^{\beta}\right]\right)= \\
& =-i \frac{D}{\hbar}\left(i \sum_{\gamma} e_{\alpha \beta \gamma} \hat{S}^{\gamma} \hat{S}^{\beta}+i \sum_{\gamma} e_{\alpha \beta \gamma} \hat{S}^{\beta} \hat{S}^{\gamma}\right)= \\
& =\frac{D}{\hbar} \sum_{\gamma} e_{\alpha \beta \gamma}\left\{\hat{S}^{\beta}, \hat{S}^{\gamma}\right\}
\end{aligned}
$$

Due to equality of axes, since there is no correspondence principle for $\left(\hat{S}^{z}\right)^{2}$, it will take place neither for $\left(\hat{S}^{x}\right)^{2}$, nor for $\left(\hat{S}^{y}\right)^{2}$.

Using this result, for a general term of both $\hat{\mathscr{H}}_{\text {anis(2) }}$ and $\hat{\mathscr{H}}_{\text {anis }(3)}: D\left(\hat{S}^{\beta}\right)^{2}\left(\hat{S}^{\chi}\right)^{2}$ (in particular, for $\beta=\chi$ we get $\hat{\mathcal{H}}_{\text {anis(3)}}$ )

we arrive at:

$$
\begin{aligned}
& \dot{\hat{S}}^{\alpha}=\frac{-i}{\hbar} D\left[\hat{S}^{\alpha},\left(\hat{S}^{\beta}\right)^{2}\left(\hat{S}^{\chi}\right)^{2}\right]=-i \frac{D}{\hbar}\left(\left[\hat{S}^{\alpha},\left(\hat{S}^{\beta}\right)^{2}\right]\left(\hat{S}^{\chi}\right)^{2}+\left(\hat{S}^{\beta}\right)^{2}\left[\hat{S}^{\alpha},\left(\hat{S}^{\chi}\right)^{2}\right]\right)= \\
& =\frac{D}{\hbar}\left(\sum_{\gamma} e_{\alpha \beta \gamma}\left\{\hat{S}^{\beta}, \hat{S}^{\gamma}\right\}\left(\hat{S}^{\chi}\right)^{2}+\left(\hat{S}^{\beta}\right)^{2} \sum_{\gamma} e_{\alpha \chi \gamma}\left\{\hat{S}^{\chi}, \hat{S}^{\gamma}\right\}\right)
\end{aligned}
$$

On the other hand in the classical situation, for $E_{\text {anis }}=D\left(S^{\beta}\right)^{2}\left(S^{x}\right)^{2}$, the anisotropy field can be represented as

$$
\begin{aligned}
& \hat{\mathcal{H}}_{\text {anis }}^{\gamma}=-\frac{D}{\left(\gamma_{s} \hbar\right)} \frac{\partial}{\partial S^{\gamma}}\left(S^{\beta}\right)^{2}\left(S^{\chi}\right)^{2}=-\frac{D}{\left(\gamma_{s} \hbar\right)}\left(2 S^{\beta} \delta_{\not \gamma}\left(S^{\chi}\right)^{2}+2\left(S^{\beta}\right)^{2} S^{\chi} \delta_{\gamma \chi}\right)= \\
& =-\frac{D}{\left(\gamma_{s} \hbar\right)}\left(2 S^{\gamma} \delta_{\gamma \beta}\left(S^{\chi}\right)^{2}+2\left(S^{\beta}\right)^{2} S^{\gamma} \delta_{\gamma \chi}\right)=-\frac{2 D}{\left(\gamma_{s} \hbar\right)} S^{\gamma}\left(\delta_{\not \beta}\left(S^{\chi}\right)^{2}+\delta_{\not \chi}\left(S^{\beta}\right)^{2}\right)
\end{aligned}
$$

Then,

$$
\begin{aligned}
& \dot{S}^{\alpha}=\gamma_{s} \sum_{v \gamma} e_{\alpha v \gamma} S^{\nu} H_{a n i s}^{\gamma}=-\frac{2 D}{\hbar} \sum_{v \gamma} e_{\alpha v \gamma} S^{v} S^{\gamma}\left(\delta_{\gamma \beta}\left(S^{\chi}\right)^{2}+\delta_{\gamma \chi}\left(S^{\beta}\right)^{2}\right)= \\
& =-\frac{2 D}{\hbar} \sum_{\nu}\left(\sum_{\gamma} e_{\alpha v \gamma} S^{v} S^{\gamma} \delta_{\gamma \beta}\left(S^{\chi}\right)^{2}+\sum_{\gamma} e_{\alpha v \gamma} S^{\nu} S^{\gamma} \delta_{\gamma \chi}\left(S^{\beta}\right)^{2}\right)= \\
& =-\frac{2 D}{\hbar} \sum_{v}\left(e_{\alpha \nu \beta} S^{\nu} S^{\beta}\left(S^{\chi}\right)^{2}+e_{\alpha \nu \chi} S^{\nu} S^{\chi}\left(S^{\beta}\right)^{2}\right) .
\end{aligned}
$$

After renaming some indexes, 


$$
\begin{aligned}
& -\frac{2 D}{\hbar} \sum_{\nu \rightarrow \gamma}\left(e_{\alpha, v \rightarrow \gamma, \beta} S^{\nu \rightarrow \gamma} S^{\beta}\left(S^{\chi}\right)^{2}+e_{\alpha, v \rightarrow \gamma, \chi} S^{\nu \rightarrow \gamma} S^{\chi}\left(S^{\beta}\right)^{2}\right)= \\
& =-\frac{2 D}{\hbar} \sum_{\gamma}\left(e_{\alpha \gamma \beta} S^{\gamma} S^{\beta}\left(S^{\chi}\right)^{2}+e_{\alpha \gamma \gamma} S^{\gamma} S^{\chi}\left(S^{\beta}\right)^{2}\right)= \\
& =\frac{2 D}{\hbar} \sum_{\gamma}\left(e_{\alpha \beta \gamma} S^{\gamma} S^{\beta}\left(S^{\chi}\right)^{2}+e_{\alpha \chi \gamma} S^{\gamma} S^{\chi}\left(S^{\beta}\right)^{2}\right) .
\end{aligned}
$$

we once again arrive just to a formal correspondence as before.

\section{b) Correspondence Principle for the Magnitude of the Total Spin in the Presence of DD-Interactions}

Obviously, the magnitude $\vec{S}^{2}$ of an individual spin is conserved for both: quantum and classical approaches. But is the magnitude of the total angular momentum conserved? Of course, it does for a system of non-interacting equal spins, though it doesn't in the case of spins with different gyromagnetic ratios (this question will be discussed in details in the next paragraph).

Now let us consider a system with dipole interactions (for simplicity we'll take two spins: $l=1,2$ ).

$$
\vec{S} \equiv \vec{S}_{1}+\vec{S}_{2}
$$

In the classical case (if we have only dipole interactions):

$$
\begin{aligned}
& \dot{\vec{S}}_{l}=\gamma_{s} \vec{S}_{l} \times \vec{H}_{l}, \\
& \vec{H}_{l}=-\gamma_{s} \overline{\bar{D}}_{l k} \vec{S}_{k} .
\end{aligned}
$$

For classical situation we use spins with dimensions of $\hbar$.

For $\frac{d}{d t}\left(\vec{S}^{2}\right)$ we get:

$$
\frac{d}{d t}\left(\vec{S}^{2}\right)=2 \vec{S} \dot{\vec{S}}=2 \vec{S}\left(\dot{\vec{S}}_{1}+\dot{\vec{S}}_{2}\right)=2 \gamma_{s} \vec{S}\left(\left[\vec{S}_{1} \times \vec{H}_{1}\right]+\left[\vec{S}_{2} \times \vec{H}_{2}\right]\right)
$$

Since $\vec{A}[\vec{B} \times \vec{C}]=[\vec{A} \times \vec{B}] \vec{C}$,

$$
\frac{d}{d t}\left(\vec{S}^{2}\right)=2 \gamma_{s}\left(\left[\vec{S} \times \vec{S}_{1}\right] \vec{H}_{1}+\left[\vec{S} \times \vec{S}_{2}\right] \vec{H}_{2}\right) \text {. }
$$

Because $\left[\vec{S} \times \vec{S}_{1}\right]=\left[\vec{S}_{1}+\vec{S}_{2} \times \vec{S}_{1}\right]=\left[\vec{S}_{2} \times \vec{S}_{1}\right]=-\left[\vec{S}_{1} \times \vec{S}_{2}\right]=-\left[\vec{S} \times \vec{S}_{2}\right]$,

$$
\frac{d}{d t}\left(\vec{S}^{2}\right)=2 \gamma_{s}\left[\vec{S}_{1} \times \vec{S}_{2}\right]\left(\vec{H}_{2}-\vec{H}_{1}\right)
$$

Taking into account the symmetry, $\overline{\bar{D}}_{21}=\overline{\bar{D}}_{12}$, we finally get: 


$$
\frac{d}{d t}\left(\vec{S}^{2}\right)=-2 \gamma_{s}\left[\vec{S}_{1} \times \vec{S}_{2}\right] \overline{\bar{D}}_{12}\left(\vec{S}_{1}-\vec{S}_{2}\right)
$$

Let us also re-write this result in tensor form, so that we can use it later for comparison with a quantum case:

$$
\begin{aligned}
& \frac{d}{d t}\left(\vec{S}^{2}\right)=-2 \gamma_{s}^{2}\left[\vec{S}_{1} \times \vec{S}_{2}\right] \overline{\bar{D}}_{12}\left(\vec{S}_{1}-\vec{S}_{2}\right)=-2 \gamma_{s}^{2} D_{12}^{\alpha \beta} e_{\beta \gamma} S_{1}^{\gamma} S_{2}^{v}\left(S_{1}^{\alpha}-S_{2}^{\alpha}\right)= \\
& =2 \gamma_{s}^{2} D_{12}^{\alpha \beta} e_{\beta \beta v} S_{1}^{\gamma} S_{2}^{v}\left(S_{1}^{\alpha}-S_{2}^{\alpha}\right)
\end{aligned}
$$

In the quantum approach:

$$
\frac{d}{d t}\left(\hat{\vec{S}}^{2}\right)=-\frac{i}{\hbar}\left[\hat{\vec{S}}^{2}, \hat{H}_{d d}\right]=-\frac{i}{\hbar}\left[\hat{\vec{S}}_{1}^{2}+\hat{\vec{S}}_{2}^{2}+2 \hat{\vec{S}}_{1} \hat{\vec{S}}_{2}, \hat{\mathscr{H}}_{d d}\right]=-2 \frac{i}{\hbar}\left[\hat{\vec{S}}_{1} \hat{\vec{S}}_{2}, \hat{H}_{d d}\right]
$$

(we took advantage of the fact that $\left[\hat{\hat{S}}_{l}^{2}, \hat{\mathscr{H}}_{d d}\right]=0$ ). Here for simplicity we again use dimensionless spins. Dimensions of $\hbar$ will be added in the end of this paragraph.

If we use expression $\hat{H}_{d d}=\frac{1}{2}\left(\gamma_{s} \hbar\right)^{2} \sum_{j k} D_{j k}^{\alpha \beta} \hat{S}_{j}^{\alpha} \hat{S}_{k}^{\beta}=\left(\gamma_{s} \hbar\right)^{2} D_{12}^{\alpha \beta} \hat{S}_{1}^{\alpha} \hat{S}_{2}^{\beta}$, and the symmetry of $D_{l k}^{\alpha \beta}$ over upper and lower indexes:

$$
\begin{aligned}
& \frac{d}{d t}\left(\hat{\vec{S}}^{2}\right)=-2 \frac{i}{\hbar}\left(\gamma_{s}^{2} \hbar^{2}\right)\left[\hat{S}_{1}^{\gamma} \hat{S}_{2}^{\gamma}, D_{12}^{\alpha \beta} \hat{S}_{1}^{\alpha} \hat{S}_{2}^{\beta}\right]=-2 i \gamma_{s}^{2} \hbar\left[\hat{S}_{1}^{\gamma} \hat{S}_{2}^{\gamma}, D_{12}^{\alpha \beta} \hat{S}_{1}^{\alpha} \hat{S}_{2}^{\beta}\right]= \\
& =-2 i \hbar \gamma_{s}^{2} D_{12}^{\alpha \beta}\left[\hat{S}_{1}^{\gamma} \hat{S}_{2}^{\gamma}, \hat{S}_{1}^{\alpha} \hat{S}_{2}^{\beta}\right]=-2 i \hbar \gamma_{s}^{2} D_{12}^{\alpha \beta}\left(\hat{S}_{1}^{\gamma}\left[\hat{S}_{2}^{\gamma}, \hat{S}_{1}^{\alpha} \hat{S}_{2}^{\beta}\right]+\left[\hat{S}_{1}^{\gamma} \hat{S}_{1}^{\alpha} \hat{S}_{2}^{\beta}\right] \hat{S}_{2}^{\gamma}\right)= \\
& =-2 i \hbar \gamma_{s}^{2} D_{12}^{\alpha \beta}\left(\hat{S}_{1}^{\gamma} \hat{S}_{1}^{\alpha}\left[\hat{S}_{2}^{\gamma}, \hat{S}_{2}^{\beta}\right]+\left[\hat{S}_{1}^{\gamma}, \hat{S}_{1}^{\alpha}\right] \hat{S}_{2}^{\beta} \hat{S}_{2}^{\gamma}\right) .
\end{aligned}
$$

Using commutation relations for spins,

$$
\begin{aligned}
& \frac{d}{d t}\left(\hat{\vec{S}}^{2}\right)=-2 i \hbar \gamma_{s}^{2} D_{12}^{\alpha \beta}\left(\hat{S}_{1}^{\gamma} \hat{S}_{1}^{\alpha} i e_{\gamma \beta v} \hat{S}_{2}^{v}+i e_{\gamma \alpha v} \hat{S}_{1}^{\gamma} \hat{S}_{2}^{\beta} \hat{S}_{2}^{\gamma}\right)= \\
& =2 \hbar \gamma_{s}^{2}\left(D_{12}^{\alpha \beta} e_{\gamma \beta \nu} \hat{S}_{1}^{\gamma} \hat{S}_{1}^{\alpha} \hat{S}_{2}^{\gamma}+D_{12}^{\alpha \beta} e_{\gamma \alpha v} \hat{S}_{1}^{v} \hat{S}_{2}^{\beta} \hat{S}_{2}^{\gamma}\right)
\end{aligned}
$$

After switching indexes $(\alpha \leftrightarrow \beta)$ in the second term

$$
\begin{aligned}
& \frac{d}{d t}\left(\hat{\vec{S}}^{2}\right)=2 \gamma_{s}^{2} \hbar\left(D_{12}^{\alpha \beta} e_{\gamma \beta \nu} \hat{S}_{1}^{\gamma} \hat{S}_{1}^{\alpha} \hat{S}_{2}^{v}+D_{12}^{\alpha \rightarrow \beta . \beta \rightarrow \alpha} e_{\gamma \cdot \alpha \rightarrow \beta, \nu} \hat{S}_{1}^{\gamma} \hat{S}_{2}^{\beta \rightarrow \alpha} \hat{S}_{2}^{\gamma}\right)= \\
& =2 \gamma_{s}^{2} \hbar\left(D_{12}^{\alpha \beta} e_{\gamma \beta \nu} \hat{S}_{1}^{\gamma} \hat{S}_{1}^{\alpha} \hat{S}_{2}^{\nu}+D_{12}^{\beta \alpha} e_{\gamma \beta \nu} \hat{S}_{1}^{\gamma} \hat{S}_{2}^{\alpha} \hat{S}_{2}^{\gamma}\right)= \\
& =2 \gamma_{s}^{2} \hbar D_{12}^{\alpha \beta} e_{\gamma \beta \nu}\left(\hat{S}_{1}^{\gamma} \hat{S}_{1}^{\alpha} \hat{S}_{2}^{v}+\hat{S}_{1}^{v} \hat{S}_{2}^{\alpha} \hat{S}_{2}^{\gamma}\right) .
\end{aligned}
$$


If we now multiply both sides of this equation by $\hbar^{2}$, we will be able to switch to spins of dimension $\hbar$ :

$$
\frac{d}{d t}\left(\hbar^{2} \hat{\vec{S}}^{2}\right)=2 \gamma_{s}^{2} D_{12}^{\alpha \beta} e_{\gamma \beta v}\left(\hbar \hat{S}_{1}^{\gamma} \hbar \hat{S}_{1}^{\alpha} \hbar \hat{S}_{2}^{v}+\hbar \hat{S}_{1}^{v} \hbar \hat{S}_{2}^{\alpha} \hbar \hat{S}_{2}^{\gamma}\right) .
$$

We can do some more transformations to bring this expression closer to the classical result:

$$
\begin{aligned}
& \frac{d}{d t}\left(\hat{\vec{S}}^{2}\right)=2 \gamma_{s}^{2} D_{12}^{\alpha \beta} e_{\gamma \beta v}\left(\hat{S}_{1}^{\gamma} \hat{S}_{1}^{\alpha} \hat{S}_{2}^{v}+\hat{S}_{1}^{\nu} \hat{S}_{2}^{\alpha} \hat{S}_{2}^{\gamma}\right)= \\
& =2 \gamma_{s}^{2} D_{12}^{\alpha \beta}\left(e_{\gamma \beta \nu} \hat{S}_{1}^{\gamma} \hat{S}_{1}^{\alpha} \hat{S}_{2}^{v}+e_{\gamma \beta v} \hat{S}_{1}^{\gamma} \hat{S}_{2}^{\alpha} \hat{S}_{2}^{\gamma}\right)= \\
& =2 \gamma_{s}^{2} D_{12}^{\alpha \beta}\left(e_{\gamma \beta \nu} \hat{S}_{1}^{\gamma} \hat{S}_{1}^{\alpha} \hat{S}_{2}^{v}+e_{\gamma \rightarrow v, \beta, v \rightarrow \gamma} \hat{S}_{1}^{\nu \rightarrow \gamma} \hat{S}_{2}^{\alpha} \hat{S}_{2}^{\gamma \rightarrow v}\right)= \\
& =2 \gamma_{s}^{2} D_{12}^{\alpha \beta}\left(e_{\gamma \beta \nu} \hat{S}_{1}^{\gamma} \hat{S}_{1}^{\alpha} \hat{S}_{2}^{v}+e_{\nu \beta \gamma} \hat{S}_{1}^{\gamma} \hat{S}_{2}^{\alpha} \hat{S}_{2}^{\nu}\right)= \\
& =2 \gamma_{s}^{2} D_{12}^{\alpha \beta}\left(e_{\gamma \beta \nu} \hat{S}_{1}^{\gamma} \hat{S}_{1}^{\alpha} \hat{S}_{2}^{v}-e_{\gamma \beta v} \hat{S}_{1}^{\gamma} \hat{S}_{2}^{\alpha} \hat{S}_{2}^{v}\right)=2 \gamma_{s}^{2} D_{12}^{\alpha \beta} e_{\gamma \beta \nu} \hat{S}_{1}^{\gamma}\left(\hat{S}_{1}^{\alpha}-\hat{S}_{2}^{\alpha}\right) \hat{S}_{2}^{v} .
\end{aligned}
$$

Now we can compare it to the classical result:

$$
\begin{aligned}
& \text { quantum: } \frac{d}{d t}\left(\hat{\vec{S}}^{2}\right)=2 \gamma_{s}^{2} D_{12}^{\alpha \beta} e_{\gamma \beta \nu} \hat{S}_{1}^{\gamma}\left(\hat{S}_{1}^{\alpha}-\hat{S}_{2}^{\alpha}\right) \hat{S}_{2}^{v} \\
& \text { classic: } \quad \frac{d}{d t}\left(\vec{S}^{2}\right)=2 \gamma_{s}^{2} D_{12}^{\alpha \beta} e_{\gamma \beta \nu} S_{1}^{\gamma} S_{2}^{\nu}\left(S_{1}^{\alpha}-S_{2}^{\alpha}\right),
\end{aligned}
$$

Thus, in the general case we get only a formal correspondence principle. Also, it is worth noticing that the magnitude of the total spin is not conserved in both cases.

The following set of graphs shows the evolution of the length of the total spin and each component of the total spin. Modeling was performed for eight spins, arranged in the shape of a cube, initial polarization is -0.6 , for a complete dipole interaction for different strength of interaction (by strength of interaction we mean $\omega_{D}=\frac{\gamma_{s}^{2} \hbar}{a^{3}}$, where $a$ is the interval between spins in the lattice). All the components of the total spin are normalized in the following way: $\sum_{j=1}^{N} \vec{S}_{j} \mapsto \frac{1}{|S| N} \sum_{j=1}^{N} \vec{S}_{j}$. Also, by polarization we mean a normalized Z-component of the total spin.

Time is measured in units of inverse Zeeman frequency. \begin{tabular}{|l|l}
$\omega_{D}=0.01$ & Normalized X,Y,Z - components of the total spin
\end{tabular} 


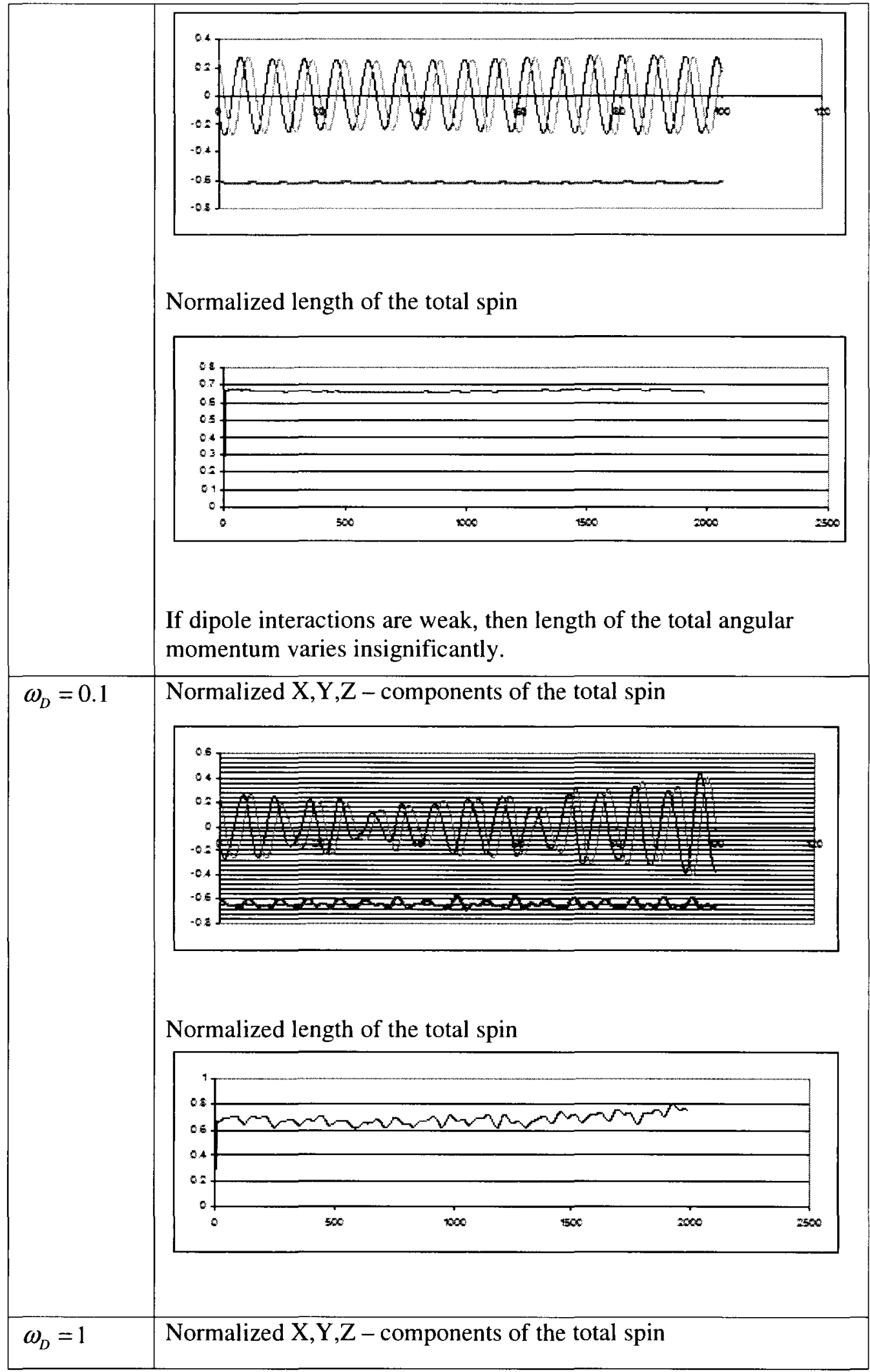




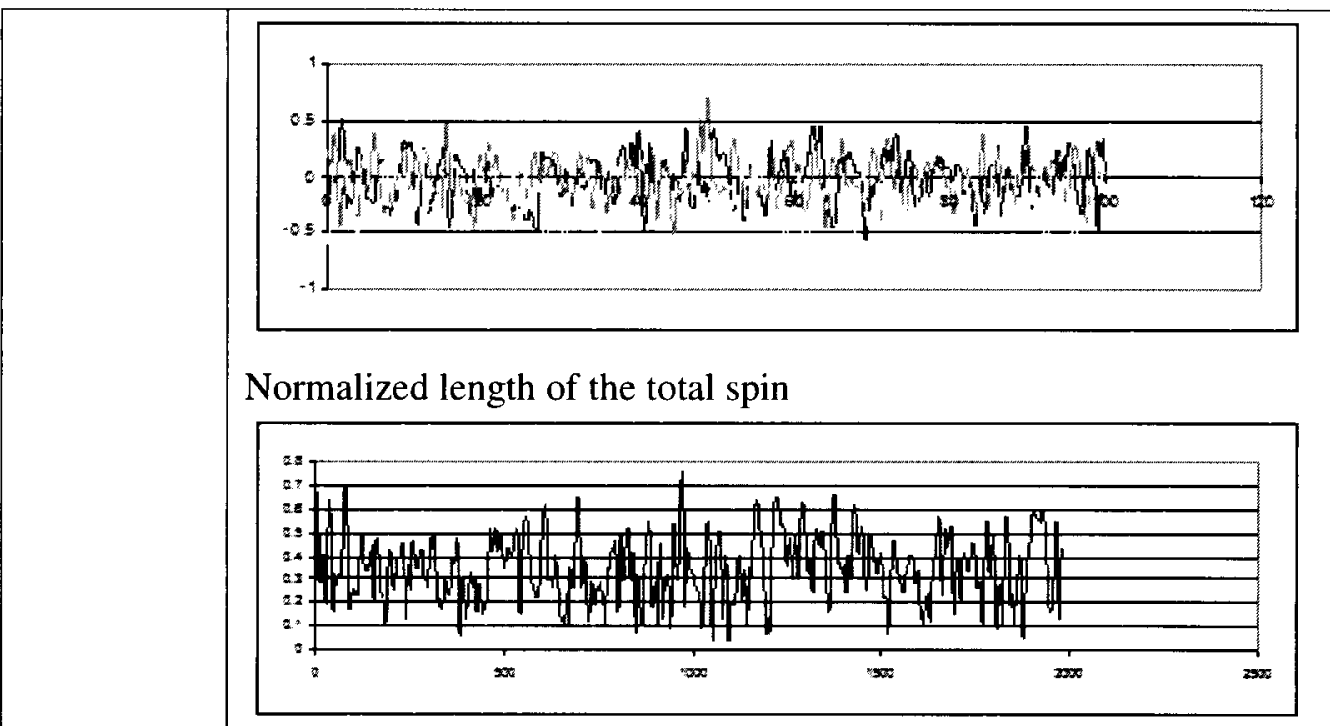

These four figures indicate that as we increase the strength of dipole interaction, variation of the total length increases as well.

Figure 1.3.b.1 time evolution of components and length of the normalized total spin in presence of dipole interactions.

c) Correspondence Principle for the Length of the Total Spin in the Case of Different Non-Interacting Particles in an External Field.

As we have pointed out in the paragraph «Correspondence principle for noninteracting spins in the external field», in the case of different $\gamma_{l}$, the length of the summary spin will not be conserved. Now let us take a look at this situation in detail:

For the quantum approach:

$$
\begin{aligned}
& \frac{d}{d t}\left(\sum_{l} \hat{S}_{l}^{\gamma}\right)^{2}=-i\left[\left(\sum_{l} \hat{S}_{l}^{\gamma}\right)^{2}, \hat{\mathcal{H}}_{0}\right]= \\
& =-i\left(\sum_{k} \hat{S}_{k}^{\gamma}\right)\left[\left(\sum_{l} \hat{S}_{l}^{\gamma}\right), \hat{H}_{0}\right]-i\left[\left(\sum_{l} \hat{S}_{l}^{\gamma}\right), \hat{H}_{0}\right]\left(\sum_{k} \hat{S}_{k}^{\gamma}\right)= \\
& =\left\{\left(\sum_{k} \hat{S}_{k}^{\gamma}\right),\left[\sum_{l} \gamma_{l} \hat{S}_{l}^{\gamma} \times \vec{H}\right]\right\} . \\
& \text { Since }\left[\sum_{k} \hat{S}_{k}^{\gamma}, \sum_{l} \gamma_{l} \hat{S}_{l}^{\gamma}\right]=\sum_{k l} \gamma_{l}\left[\hat{S}_{k}^{\gamma}, \hat{S}_{l}^{\gamma}\right]=0,
\end{aligned}
$$




$$
\frac{d}{d t}\left(\sum_{l} \hat{S}_{l}^{\gamma}\right)^{2}=\left\{\left(\sum_{k} \hat{S}_{k}^{\gamma}\right),\left[\sum_{l} \gamma_{l} \hat{S}_{l}^{\gamma} \times \vec{H}\right]\right\}=2\left(\sum_{k} \hat{S}_{k}^{\gamma}\right)\left[\sum_{l} \gamma_{l} \hat{S}_{l}^{\gamma} \times \vec{H}\right]
$$

For the classical approach:

$$
\begin{aligned}
& \frac{d}{d t}\left(\sum_{l} S_{l}^{\gamma}\right)^{2}=2\left(\sum_{k} S_{k}^{\gamma}\right) \frac{d}{d t}\left(\sum_{l} S_{l}^{\gamma}\right)= \\
& =\left(\sum_{k} \vec{S}_{k}\right)\left[\sum_{l} \gamma_{l} \vec{S}_{l} \times \vec{H}\right]=\left(\sum_{k} \vec{S}_{k}, \sum_{l} \gamma_{l} \vec{S}_{l}, \vec{H}\right) \neq 0 .
\end{aligned}
$$

Thus, the correspondence principle takes place, but the law of conservation is true only for equal $\gamma_{l}:\left(\sum_{k} \vec{S}_{k}, \sum_{l} \gamma_{l} \vec{S}_{l}, \vec{H}\right)=\left(\sum_{k} \vec{S}_{k}, \gamma_{s} \sum_{l} \vec{S}_{l}, \vec{H}\right)=0$. Otherwise, the length of the total momentum is not conserved.

The following diagrams show the evolution of total angular momentum of the same system as before ( 8 spins, arranged in the shape of a cube, initial polarization is -0.6 , no interactions):

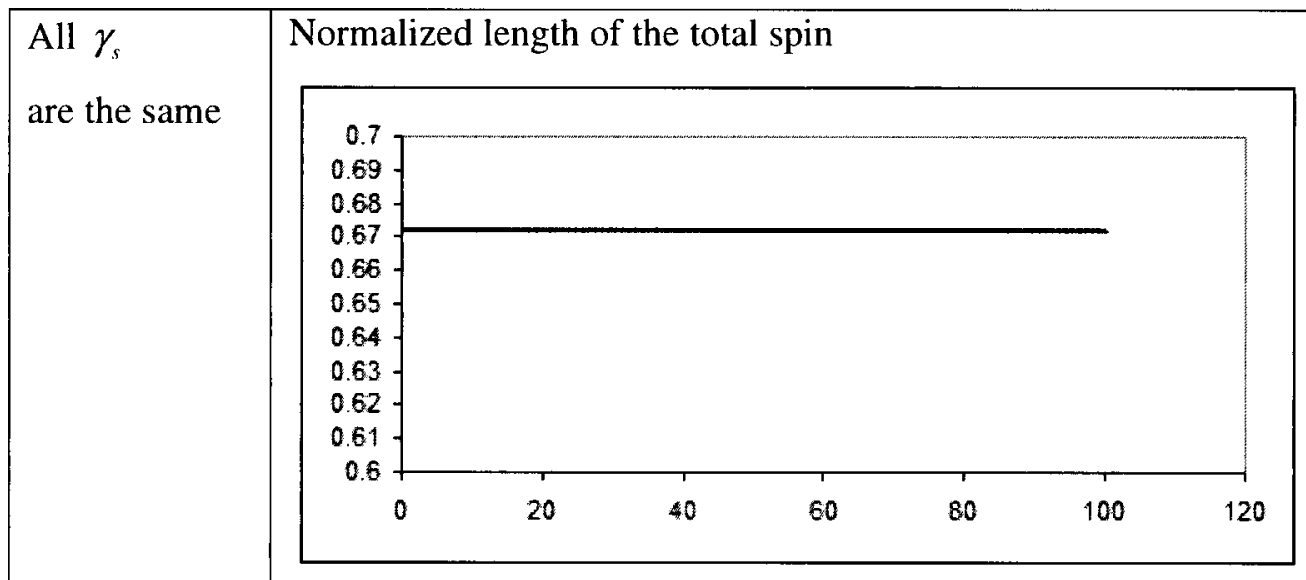

As predicted, in case of equal gyromagnetic ratios, system of many spins behaves as a single angular momentum with constant length. 


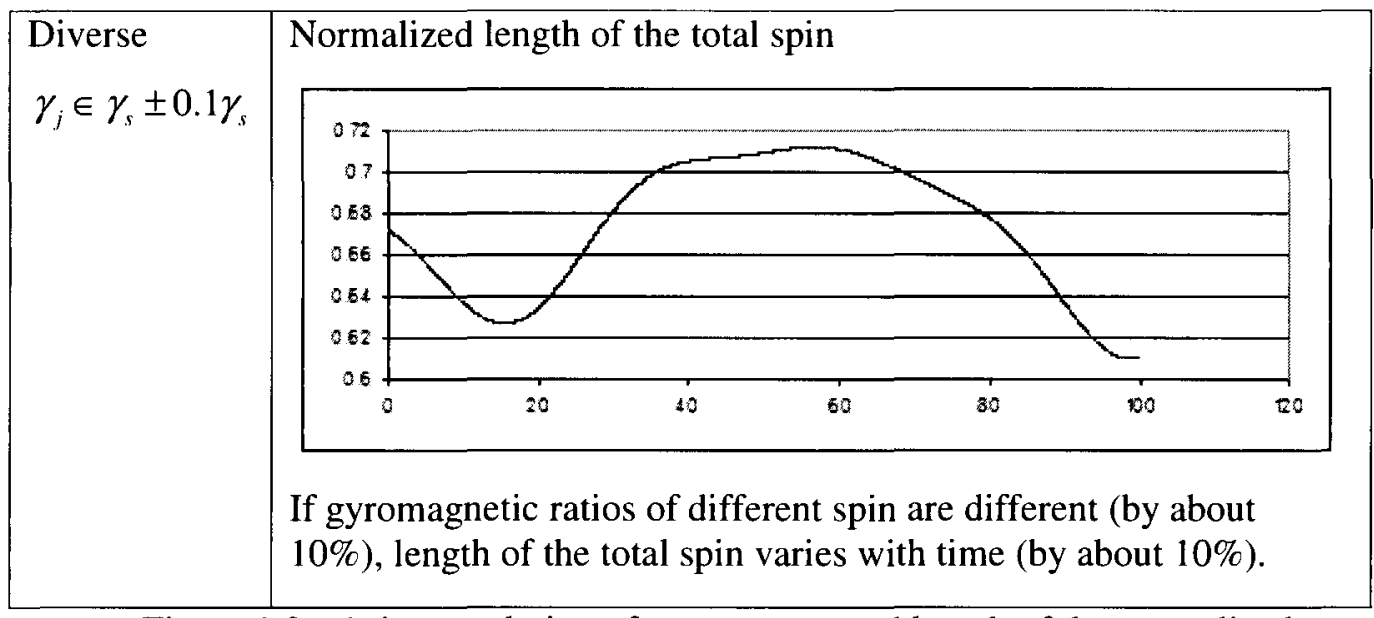

Figure 1.3.c. 1 time evolution of components and length of the normalized total spin in case of different gyrommagnetic ratios 


\section{QUANTUM SYSTEM OF EQUATIONS. SPLITTING OF THE HAMILTONIAN INTO SECULAR AND NON-SECULAR TERMS. CORRESPONDENCE PRINCIPLE FOR THE SECULAR TERMS.}

In strong fields, the Zeeman term is much larger than all the other terms. It is possible to get rid of large terms, if we switch to the rotating frame (further called RF). In order to do this we have to split the Hamiltonian into Secular and Non-secular terms. Unlike many traditional NMR problems due to the feedback via the resonator, we will not be able to get rid of fast oscillating terms. Nevertheless, analyzing the Secular Hamiltonian might bring us to some conclusions about consequences of rejection the Non-secular term.

The dipole-dipole interaction Hamiltonian can be represented as the sum of secular and non-secular terms, respectively:

$$
\hat{\mathscr{H}}_{d d}=\hat{\mathscr{H}}_{d d}^{\prime}+\hat{\mathscr{H}}_{d d}^{\prime \prime}
$$

Splitting into secular (terms with $a_{j k}$ ) and Non-secular terms is performed in the following way:

$$
\begin{aligned}
& \hat{H}_{d d}=\frac{(\gamma \hbar)^{2}}{2} \sum_{j k} D_{j k}^{\alpha \beta} \hat{S}_{j}^{\alpha} \hat{S}_{k}^{\beta}= \\
& =\frac{\mu_{0}^{2}}{2} \sum_{j k}\left\{a_{j k}\left[\hat{S}_{j}^{z} \hat{S}_{k}^{z}-\frac{1}{2} \hat{S}_{j}^{+} \hat{S}_{k}^{-}\right]+b_{j k} \hat{S}_{j}^{+} \hat{S}_{k}^{+}+b_{j k}^{*} \hat{S}_{j}^{-} \hat{S}_{k}^{-}+2 c_{j k} \hat{S}_{j}^{+} \hat{S}_{k}^{z}+2 c_{j k}^{*} \hat{S}_{j}^{-} \hat{S}_{k}^{z}\right\} \\
& \hat{H}_{d d}^{\prime}=\frac{(\gamma \hbar)^{2}}{2} \sum_{j k} D_{j k}^{\alpha \beta} \hat{S}_{j}^{\alpha} \hat{S}_{k}^{\beta}=\frac{\mu_{0}^{2}}{2} \sum_{j k} a_{j k}\left[\hat{S}_{j}^{z} \hat{S}_{k}^{z}-\frac{1}{4}\left(\hat{S}_{j}^{+} \hat{S}_{k}^{-}+\hat{S}_{j}^{-} \hat{S}_{k}^{+}\right)\right]
\end{aligned}
$$

Taking into account the symmetry of $a_{j k}$,

$$
\hat{H}_{d d}^{\prime}=\frac{(\gamma \hbar)^{2}}{2} \sum_{j k} a_{j k}\left[\hat{S}_{j}^{z} \hat{S}_{k}^{z}-\frac{1}{2} \hat{S}_{j}^{+} \hat{S}_{k}^{-}\right] \text {, where } a_{l k}=D_{l k}^{z z} \text {. }
$$

All the other coefficients can be also defined in terms of $D_{l k}^{\alpha \beta}$ :

$$
b_{l k}=\frac{1}{4}\left(D_{l k}^{x x}-D_{l k}^{y z}-2 i D_{l k}^{x y}\right), \quad c_{l k}=\frac{1}{2}\left(D_{l k}^{x z}-i D_{l k}^{y z}\right) \text {. }
$$


As we have indicated before, the correspondence principle takes place for any symmetric $D_{l k}^{\alpha \beta}$.

In the previous part we got equations for $\dot{\hat{\vec{\mu}}}_{d d(l)}$ in the case of complete Hamiltonian.

Now if we switch our Hamiltonian to a truncated one, then the equation for $\dot{\hat{\vec{\mu}}}_{d d(l)}$ will change in the following way:

$$
\dot{\hat{\vec{\mu}}}_{d d(l)}^{\prime}=\frac{-i}{\hbar}\left[\dot{\hat{\vec{\mu}}}_{d d(l)}, \hat{H}_{d}^{\prime}\right]
$$

Here $\hat{\vec{\mu}}_{d d(l)}^{\prime}$ means magnetic moment operator, calculated only with the secular Hamiltonian.

To find $\dot{\hat{\mu}}_{d d(l)}^{\prime}$, we have to analyze the secular Hamiltonian:

$$
\begin{aligned}
& \hat{\mathcal{H}}_{d d}^{\prime}=\frac{1}{2}(\gamma \hbar)^{2} \sum_{j>k} D_{j k}^{z z}\left(2 S_{j}^{z} S_{k}^{z}-S_{j}^{x} S_{k}^{x}-S_{j}^{y} S_{k}^{y}\right)= \\
& =\frac{1}{4}(\gamma \hbar)^{2} \sum_{j \neq k} D_{j k}^{z z}\left(2 S_{j}^{z} S_{k}^{z}-S_{j}^{x} S_{k}^{x}-S_{j}^{y} S_{k}^{y}\right)
\end{aligned}
$$

This can be rewritten in the following way:

$$
\hat{H}_{d}^{\prime}=\frac{1}{2}(\gamma \hbar)^{2} \sum_{j \neq k} \tilde{D}_{j k}^{\alpha \beta} \hat{S}_{j}^{\alpha} \hat{S}_{k}^{\beta} \text {, }
$$

where

$$
\tilde{D}_{j k}^{\alpha \beta}=\left(\begin{array}{ccc}
-\frac{1}{2} D_{j k}^{33} & 0 & 0 \\
0 & -\frac{1}{2} D_{j k}^{33} & 0 \\
0 & 0 & D_{j k}^{33}
\end{array}\right)
$$

This means that switching from the complete Hamiltonian to a truncated one is equivalent to change of the tensor $D_{j k}^{\alpha \beta}$ by $\tilde{D}_{j k}^{\alpha \beta}$, which has the same symmetry. Thus, the expression for $\dot{\hat{\vec{\mu}}}_{d d(l)}^{\prime}$ will be formally equivalent to the one we got earlier for the complete Hamiltonian. The only difference is that the dipolar field will be calculated as

$$
\hat{\tilde{H}}_{d d(l)}^{\beta}=-\sum_{j \neq l} \tilde{D}_{j l}^{\alpha \beta} \hat{\mu}_{l}^{\alpha}
$$

Since $\tilde{D}_{j k}^{\alpha \beta}$ has only diagonal elements, in the model with the truncated Hamiltonian the $\alpha$-th component of $\hat{\mu}_{l}^{\alpha}$ will create only the $\alpha$-th component of the dipole field. In other words, only $x-x, y-y, z-z$ kinds of interactions will take place. The absence of mixed interactions ( $x-y, x-z, y-z)$ leads to the fact that if the state vector of the system was initially factorizable, it will always remain such. 


\section{Commutation of the Z-Component of Spins With the Secular Hamiltonian.}

Let us consider the secular part of the dipole-dipolar interaction Hamiltonian:

$$
\begin{aligned}
& \hat{H}_{d d}^{\prime}=\frac{1}{2}\left(\gamma_{s} \hbar\right)^{2} \sum_{j>k} D_{j k}^{z z}\left(2 \hat{S}_{j}^{z} \hat{S}_{k}^{z}-\hat{S}_{j}^{x} \hat{S}_{k}^{x}-\hat{S}_{j}^{y} \hat{S}_{k}^{y}\right)= \\
& =\frac{1}{4}\left(\gamma_{s} \hbar\right)^{2} \sum_{j \neq k} D_{j k}^{z z}\left(2 \hat{S}_{j}^{z} \hat{S}_{k}^{z}-\hat{S}_{j}^{x} \hat{S}_{k}^{x}-\hat{S}_{j}^{y} \hat{S}_{k}^{y}\right)= \\
& =\frac{1}{4}\left(\gamma_{s} \hbar\right)^{2} \sum_{j \neq k} D_{j k}^{z z}\left(3 \hat{S}_{j}^{z} \hat{S}_{k}^{z}-\hat{\hat{S}}_{j} \hat{\bar{S}}_{k}\right) .
\end{aligned}
$$

Now introduce the notation $\sum_{j \neq k} \hat{\mathcal{H}}_{d d(j k)}^{\prime}$, such that

$$
\hat{\mathcal{H}}_{d d}^{\prime} \equiv \sum_{j \neq k} \hat{\mathcal{H}}_{d d(j k)}^{\prime}
$$

i.e.

$\hat{\mathcal{H}}_{d d(j k)}^{\prime}=\frac{1}{4}\left(\gamma_{s} \hbar\right)^{2} D_{j k}^{z z}\left(3 \hat{S}_{j}^{z} \hat{S}_{k}^{z}-\hat{\vec{S}}_{j} \hat{\vec{S}}_{k}\right)$.

Let us show that $\hat{H}_{d d}^{\prime}$ commutes with $\hat{S}^{z} \equiv \sum_{j} \hat{S}_{j}^{z}$.

Let us first consider a system of two particles ( $\mathrm{j}$ and $\mathrm{k})$ and show that $H_{d d(j \mathrm{k})}^{\prime}$ commutes with $\hat{S}_{j}^{z}+\hat{S}_{k}^{z}$.

For this purpose we can consider the operator $\left(\hat{\vec{S}}_{j}+\hat{\vec{S}}_{k}\right)^{2}=\left(\hat{\vec{S}}_{j}\right)^{2}+\left(\hat{\vec{S}}_{k}\right)^{2}+2 \hat{\vec{S}}_{j} \hat{\vec{S}}_{k}$, which commutes with $\hat{S}_{j}^{z}+\hat{S}_{k}^{z}$.

Since $S_{j}^{z}+S_{k}^{z}$ commutes with each term in the right-hand side of

$$
\begin{aligned}
\hat{\vec{S}}_{j} \hat{\vec{S}}_{k}= & \frac{1}{2}\left(\left(\hat{\vec{S}}_{j}+\hat{\vec{S}}_{k}\right)^{2}-\left(\hat{\vec{S}}_{j}\right)^{2}-\left(\hat{\vec{S}}_{k}\right)^{2}\right) \text {, then } \\
& {\left[\hat{\vec{S}}_{j} \hat{\vec{S}}_{k}, \hat{S}_{j}^{z}+\hat{S}_{k}^{z}\right]=0 . }
\end{aligned}
$$

Since $\hat{S}_{j}^{z}+\hat{S}_{k}^{z}$ commutes with both $S_{j}^{z} S_{k}^{z}$ and $\vec{S}_{j} \vec{S}_{k}$, from equation (2.1.2) one can see that

$$
\left[\hat{\mathcal{H}}_{d d(j k)}^{\prime}, \hat{S}_{j}^{z}+\hat{S}_{k}^{z}\right]=0 \text {. }
$$

But

$$
\left[\hat{H}_{d d(j k)}^{\prime \prime}, \hat{S}_{j}^{z}+\hat{S}_{k}^{z}\right] \neq 0
$$


Now let us switch to the general case.

Let's consider a commutator $\left[\hat{\mathcal{H}}_{d d}^{\prime}, \hat{S}^{z}\right]$ :

$$
\left[\hat{\mathcal{H}}_{d d}^{\prime}, \hat{S}^{z}\right]=\left[\sum_{j \neq k} \hat{\mathcal{H}}_{d d(j k)}^{\prime}, \sum_{l} \hat{S}_{l}^{z}\right]=\sum_{j \neq k} \sum_{l}\left[\hat{H}_{d d(j k)}^{\prime}, \hat{S}_{i}^{z}\right] \quad \text { (2.1.5). }
$$

Since $\left[\hat{\mathcal{H}}_{d d(j k)}^{\prime}, \hat{S}_{i}^{z}\right]=0$, if $l \neq k \wedge l \neq j$, then nothing will change, if we

multiply this commutator by $\left(\delta_{l j}+\delta_{l k}\right)$ :

$$
\left[H_{d d(j k)}^{\prime}, S_{l}^{z}\right]=\left[H_{d d(j k)}^{\prime}, S_{l}^{z}\right]\left(\delta_{l j}+\delta_{l k}\right) \text {. }
$$

After substituting this expression into (2.1.5), then after simple transformations:

$$
\begin{aligned}
& {\left[\hat{\mathscr{H}}_{d d}^{\prime}, \hat{S}^{z}\right]=\sum_{j \neq k} \sum_{l}\left[\hat{\mathscr{H}}_{d d}^{\prime}, \hat{S}_{l}^{z}\right]\left(\delta_{l j}+\boldsymbol{\delta}_{l k}\right)=} \\
& =\sum_{j \neq k}\left[\hat{\mathscr{H}}_{d d}^{\prime}, \hat{S}_{j}^{z}\right]+\sum_{j \neq k}\left[\hat{\mathcal{H}}_{d d}^{\prime}, \hat{S}_{k}^{z}\right]= \\
& =\sum_{j \neq k}\left[\hat{\mathscr{H}}_{d d(j k)}^{\prime}, \hat{S}_{j}^{z}+\hat{S}_{k}^{z}\right] .
\end{aligned}
$$

According to (2.1.4), the commutator inside the summation sign is equal to zero, and consequently:

$$
\left[\hat{\mathcal{H}}_{d d}^{\prime}, \hat{S}^{z}\right]=0 \text {. }
$$

It is worth noticing, that this result does not depend on the shape of a sample and remains true even for partial sums (i.e. if all the summations are performed not over all the particles, but only over some part of them) - for example for clusters and for diluted systems.

Thus, if we leave only the secular term, as it is often done, dipole interactions will not bring any contribution to the $z$-component of the total spin.

The following figures show that as predicted, the Z-component of the total spin will be conserved for the secular Hamiltonian, whereas the length of the total spin will change with time:

On the following figures we have the same system as before $(8$ spins, arranged in the shape of a cube, initial polarization is -0.6 , secular dipole interactions): Due to this the length of the total spin changes less than for complete interactions. The Zcomponent of the total spin remains constant.

\begin{tabular}{|l|l}
\hline$\omega_{D}=0.01$ & Normalized $X, Y, Z-$ components of the total spin \\
\hline
\end{tabular} 


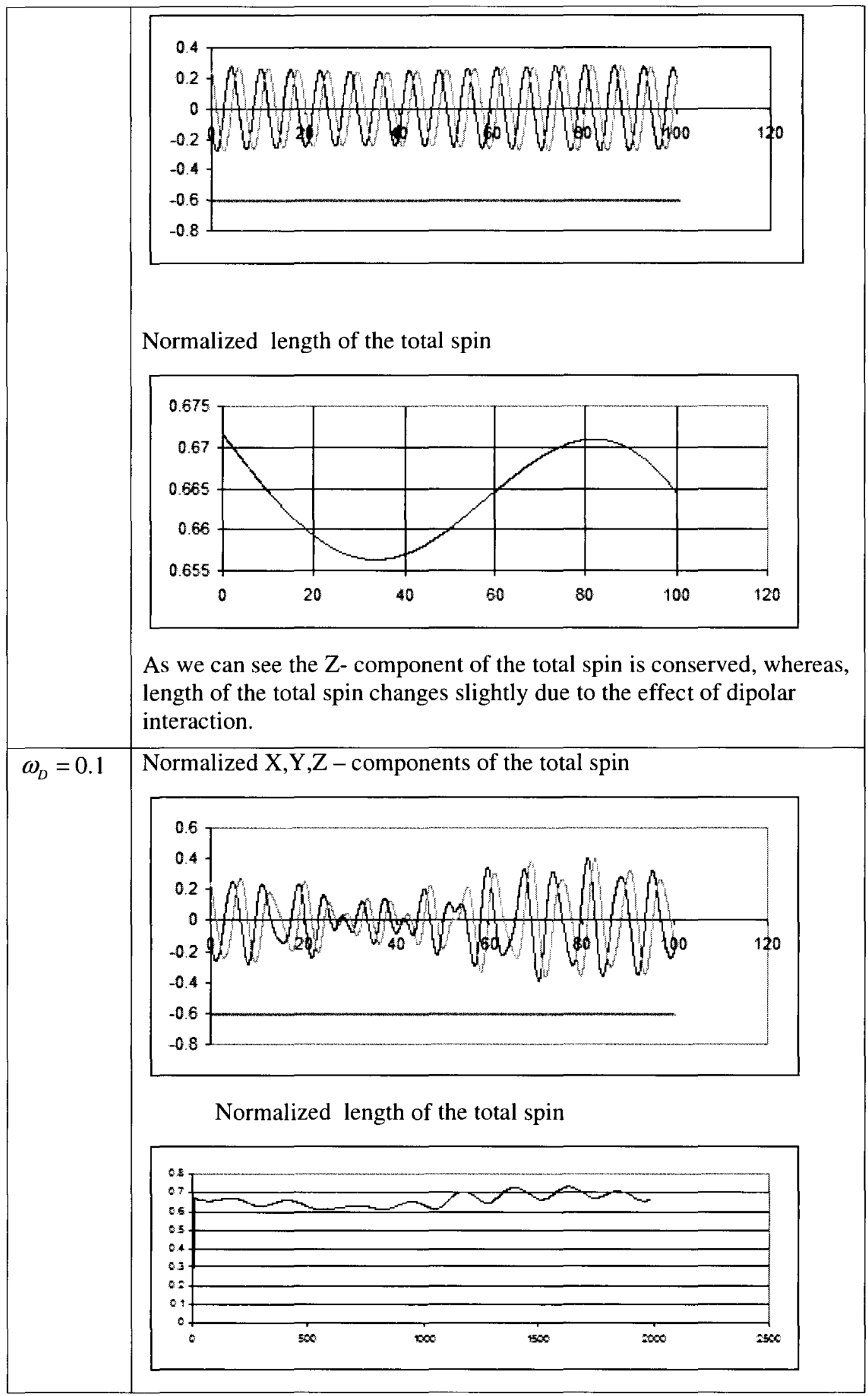




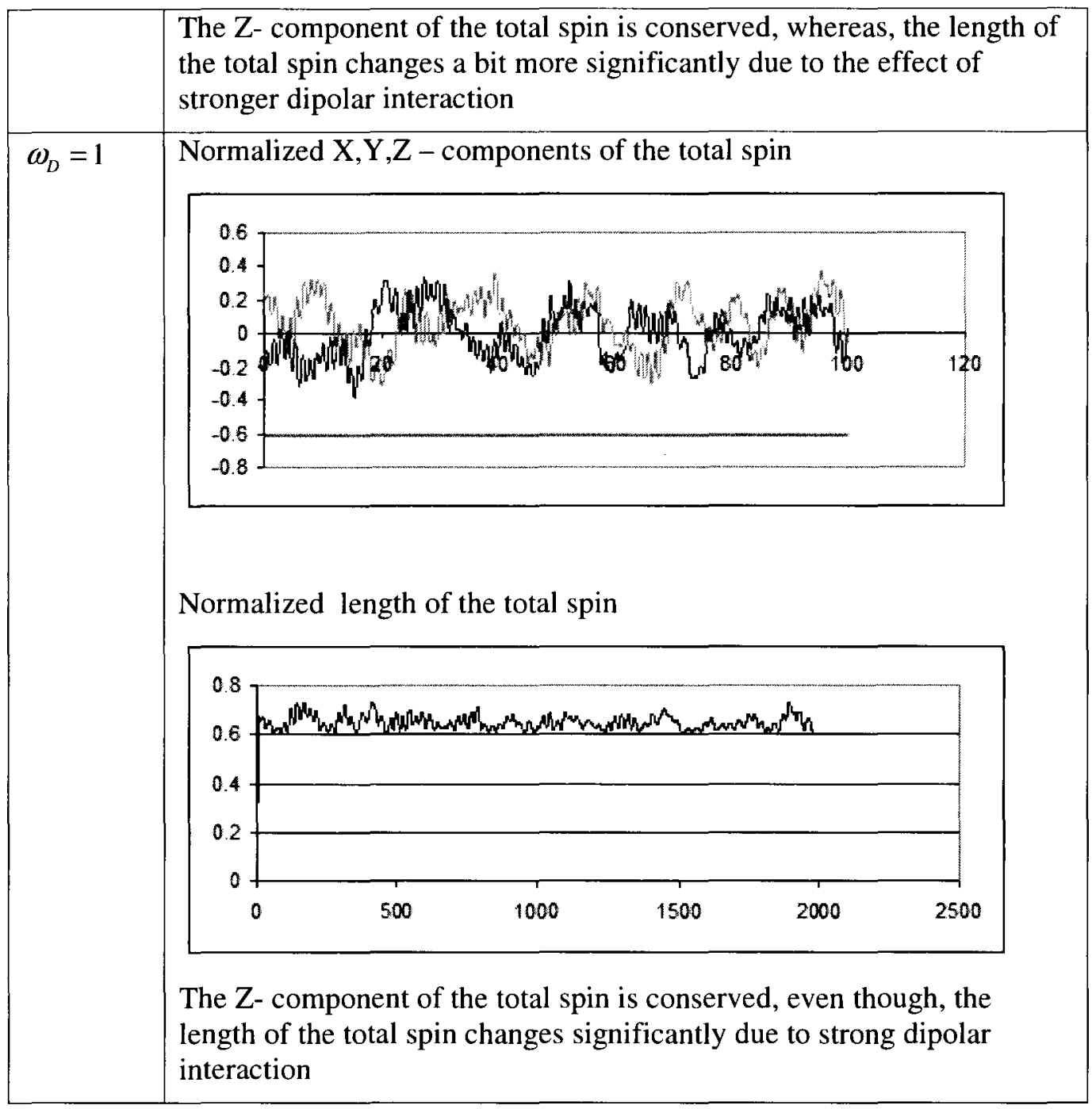

Figure 2.1.1 time evolution of the Z-component of the total spin in case of secular dipolar interactions.

Even though for strong dipole interactions the length of the total spin changes, the Z-component remains constant.

\section{Omission of Non-Secular Terms.}

The reason due to which one can leave only the secular term of the Hamiltonian, is that non-secular Hamiltonian does not contribute to the first-order of the perturbation theory in the case where DD-interactions are small with respect to the Zeeman term. It can be seen from the following considerations. Consider the 
Hamilton $-\vec{B}\left(\gamma_{s} \hbar\right) \hat{\vec{S}}+\hat{H}_{d d}^{\prime}$ unperturbed (for simplicity we'll use a non-degenerate case). For $\vec{B} \| O z$ :

$$
\left[-\vec{B}\left(\gamma_{s} \hbar\right) \hat{\vec{S}}+\hat{\mathscr{H}}_{d d}^{\prime}, \hat{\mathcal{H}}_{d d}^{\prime}\right]=-\left(\gamma_{s} \hbar\right) B_{z}\left[\hat{S}^{z}, \hat{\mathcal{H}}_{d d}^{\prime}\right]=0 \text {. }
$$

Consequently, $\hat{S}^{z}$ and $\hat{H}^{\prime}$ have common eignstates:

$$
\left\{\begin{array}{l}
\hat{S}^{z}\left|j_{0}\right\rangle=S_{j}^{z}\left|j_{0}\right\rangle \\
\left(-\vec{B}\left(\gamma_{s} \hbar\right) \hat{\vec{S}}+\hat{\mathcal{H}}_{d d}^{\prime}\right)\left|j_{0}\right\rangle=E_{j}\left|j_{0}\right\rangle
\end{array}\right.
$$

Then, if we treat $\hat{\mathcal{H}}_{d d}^{\prime \prime}$ as a small perturbation, for first order of perturbation theory:

$$
\begin{aligned}
& \left(-\vec{B}\left(\gamma_{s} \hbar\right) \hat{\vec{S}}+\hat{\mathcal{H}}_{d d}\right)|j\rangle=\left(\left\{-\vec{B}\left(\gamma_{s} \hbar\right) \hat{\vec{S}}+\hat{\mathcal{H}}_{d d}^{\prime}\right\}+\hat{\mathcal{H}}_{d d}^{\prime \prime}\right)|j\rangle=\left(E_{j}+E_{j}^{\prime \prime}\right)|j\rangle, \\
& E_{j}^{\prime \prime}=\left\langle j_{0}\left|\hat{\mathcal{H}}_{d d}^{\prime \prime}\right| j_{0}\right\rangle .
\end{aligned}
$$

Since $\hat{H}_{d d}^{\prime \prime}$ is not diagonal, $E_{j}^{\prime \prime}=\left\langle j_{0}\left|\hat{H}_{d d}^{\prime \prime}\right| j_{0}\right\rangle=0$. Thus, $\hat{H}_{d d}^{\prime \prime}$ can contribute only starting as a second order perturbation. Thus, the non-secular energy contribution will be of order of $\Delta E^{\prime \prime}\left[\frac{\left(\hbar \omega_{D}\right)^{2}}{\hbar \omega_{0}}=\left(\frac{\omega_{D}}{\omega_{0}}\right) \hbar \omega_{D}\right.$. That means that contribution of non-secular terms can be only observed at times of order $t \square \frac{1}{\Delta E^{\prime \prime} / \hbar}=\left(\frac{\omega_{0}}{\omega_{D}}\right) \frac{1}{\omega_{D}}$, where $\omega_{0}$ is the Zeeman frequency and $\omega_{D}$ corresponds to the energy of the dipole-dipole interactions.

The following figures demonstrate how, due to the correspondence principle, we can get precise results for energies using the model of classical spins. $\mathrm{B} \| \mathrm{z}$.

On the following diagram one can see the system of spins in the Zeeman field

The vectors between all the particles $(1-2,1-3,1-4,2-3,2-4, \ldots)$ have the same angle with respect to the $\mathrm{Z}$-axis. The secular Hamiltonian can be written in spherical coordinates in the form

$$
\begin{aligned}
& \hat{H}_{d d}^{\prime}=\frac{1}{2} \sum_{j \neq k} \hat{H}_{d d(j k)}^{\prime}, \text { where } \\
& \hat{H}_{d d(j k)}^{\prime}=\frac{1}{2}\left(3 \cos ^{2} \vartheta_{j k}-1\right)\left(3 \hat{S}_{j}^{z} \hat{S}_{k}^{z}-\hat{\hat{S}}_{j} \hat{\vec{S}}_{k}\right)
\end{aligned}
$$

Since all the particles are aligned so that, $\vartheta_{j k}=\vec{R}_{j k} \hat{,} \vec{B} ; \forall j, k\left(\vec{R}_{j k}\right.$ is a radius vector between j-th and k-th particles); $\vartheta_{j k}=\vartheta_{12}=\vartheta_{23}=\vartheta_{14}=\ldots$, if we set the angle 
$\vartheta_{j k}=\arccos \frac{1}{\sqrt{3}} \approx 54.7^{\circ}$ (magic angle), then $3 \cos ^{2} \vartheta_{j k}-1=0$, and consequently, all the $H_{j k}^{\prime}=0$. Then the secular Hamiltonian completely vanishes.

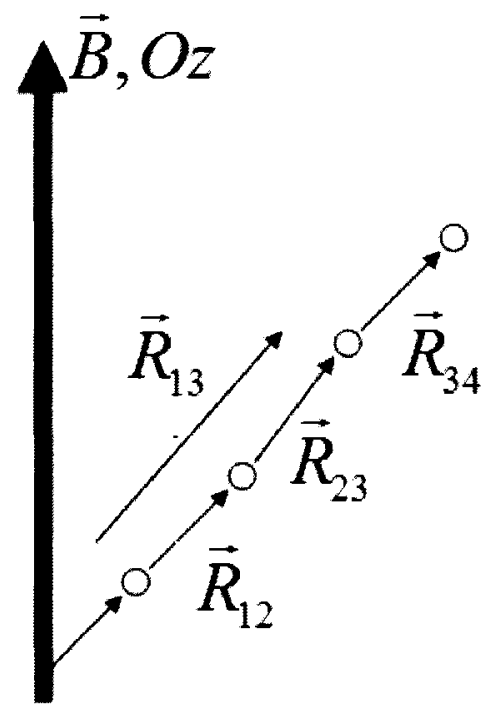

Figure 2.2.1 system of spins, arranged in the "magic angle" direction

On the following diagrams one can see the difference between the two cases (with magic angle and when spins are aligned along $\mathrm{X}$-axis) and between Secular and non-secular terms of the Hamiltonian (in the magic angle case we can observe the contribution of non-secular term if we set big-enough times $\left.t \square\left(\frac{\omega_{0}}{\omega_{D}}\right) \frac{1}{\omega_{D}}\right)$.

The following set of figures (time evolution of normalized $\mathrm{X}$ and $\mathrm{Z}$ components of the total spin) is generated by our program for the system of eight spins aligned in different directions with different strength of dipole interaction. Here we use a unitless system, where $\omega_{0}=1$. We will be altering strength of the dipole interaction $\omega_{D}$ and rearrange spins in the X-direction (in this case both secular and non-secular Hamiltonians are active) and in the magic-angle direction (only nonsecular interaction takes place). Comments in italic font will help to illustrate the relationship of the strengths of secular and non-secular interactions. We will be estimating the influence of dipole interaction by looking at the time which it takes for this kind of interaction to be observed. 


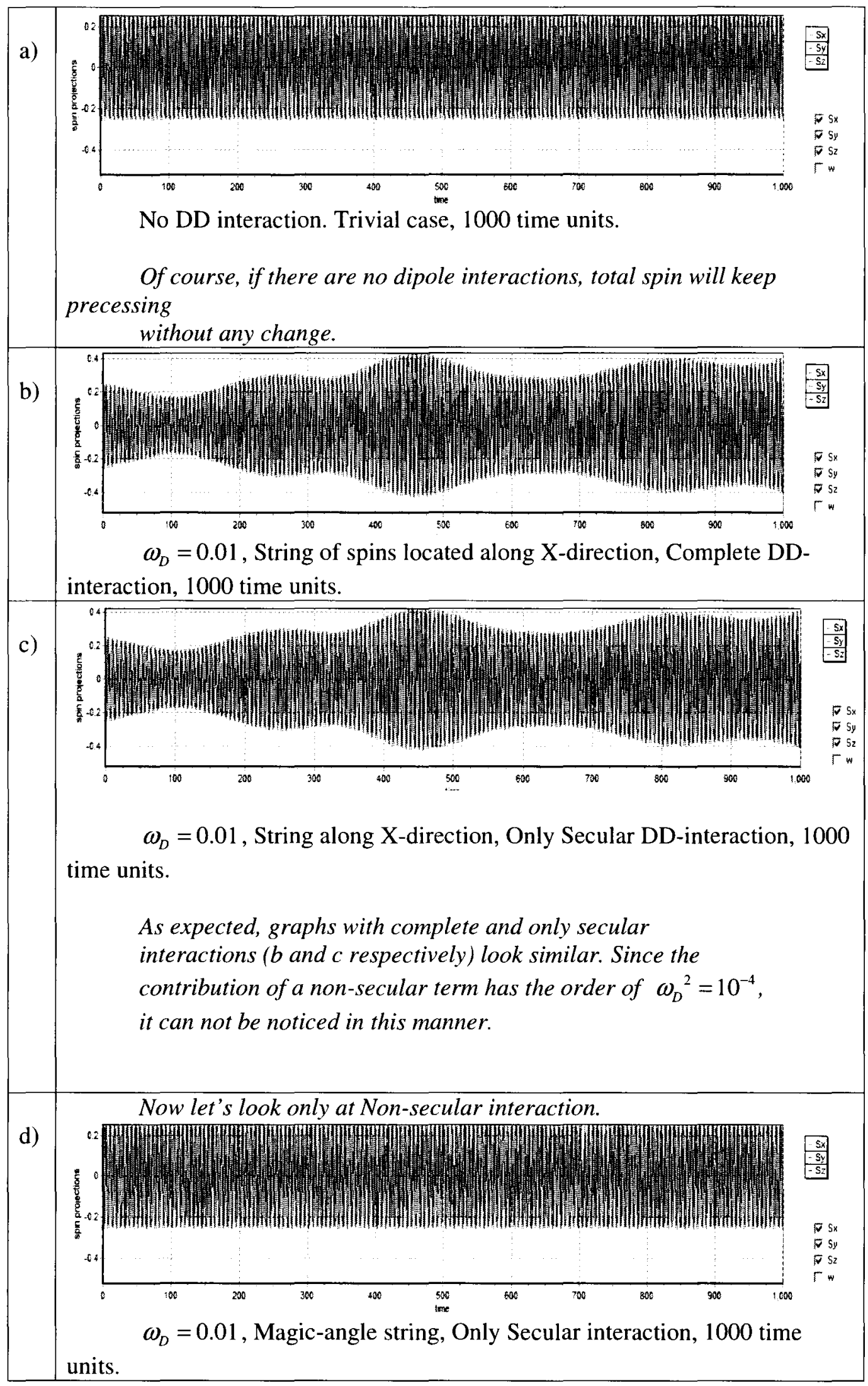




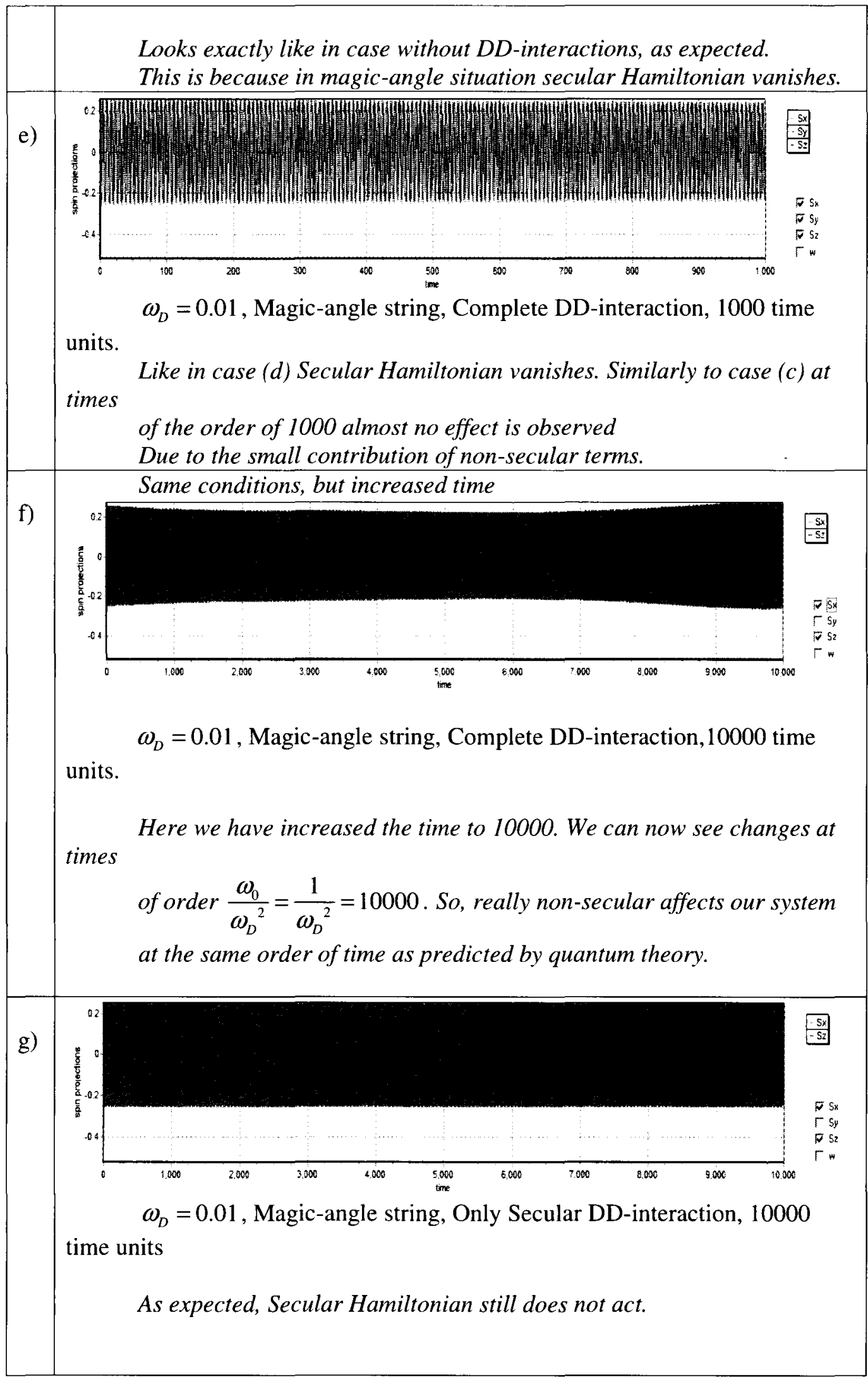




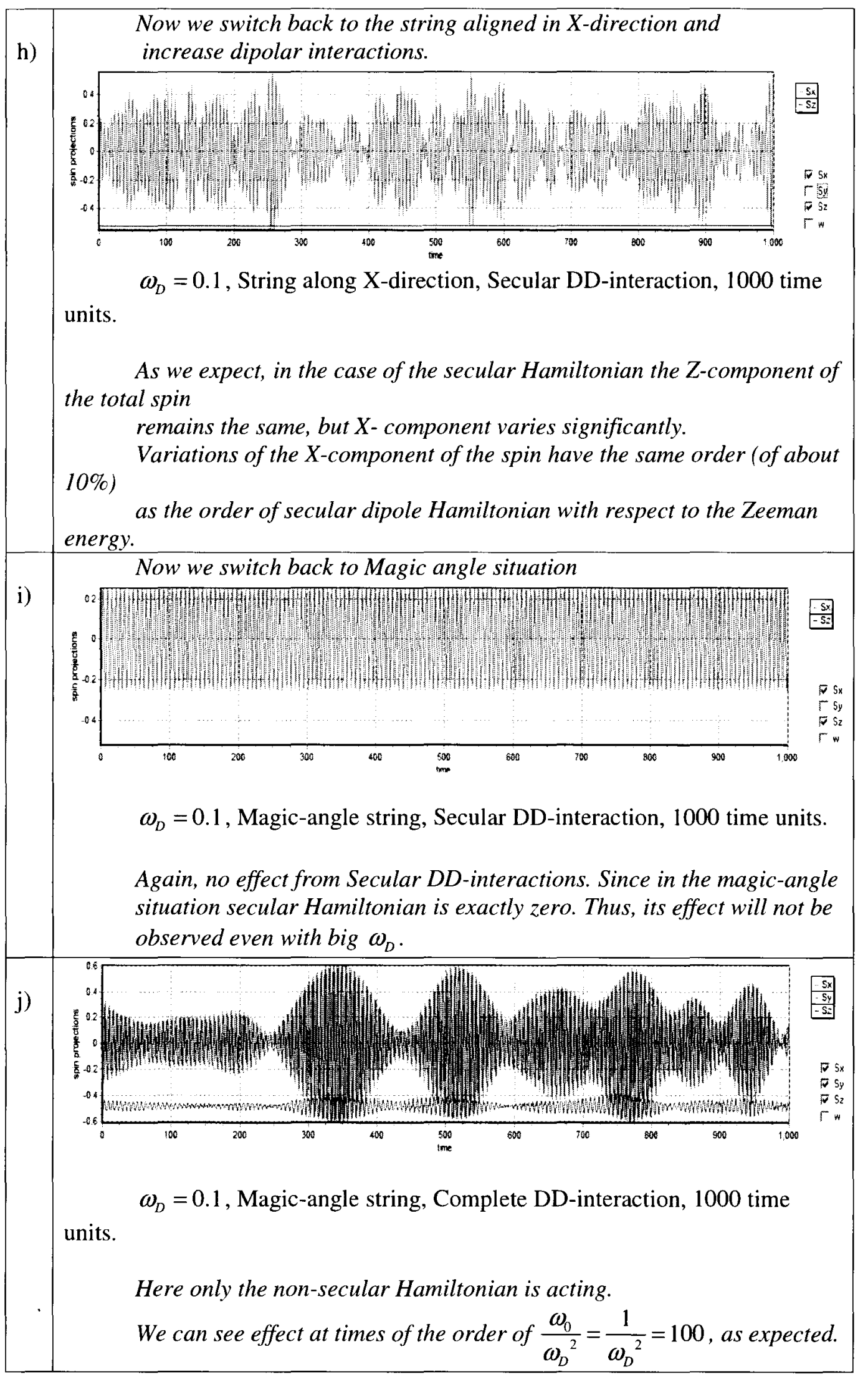




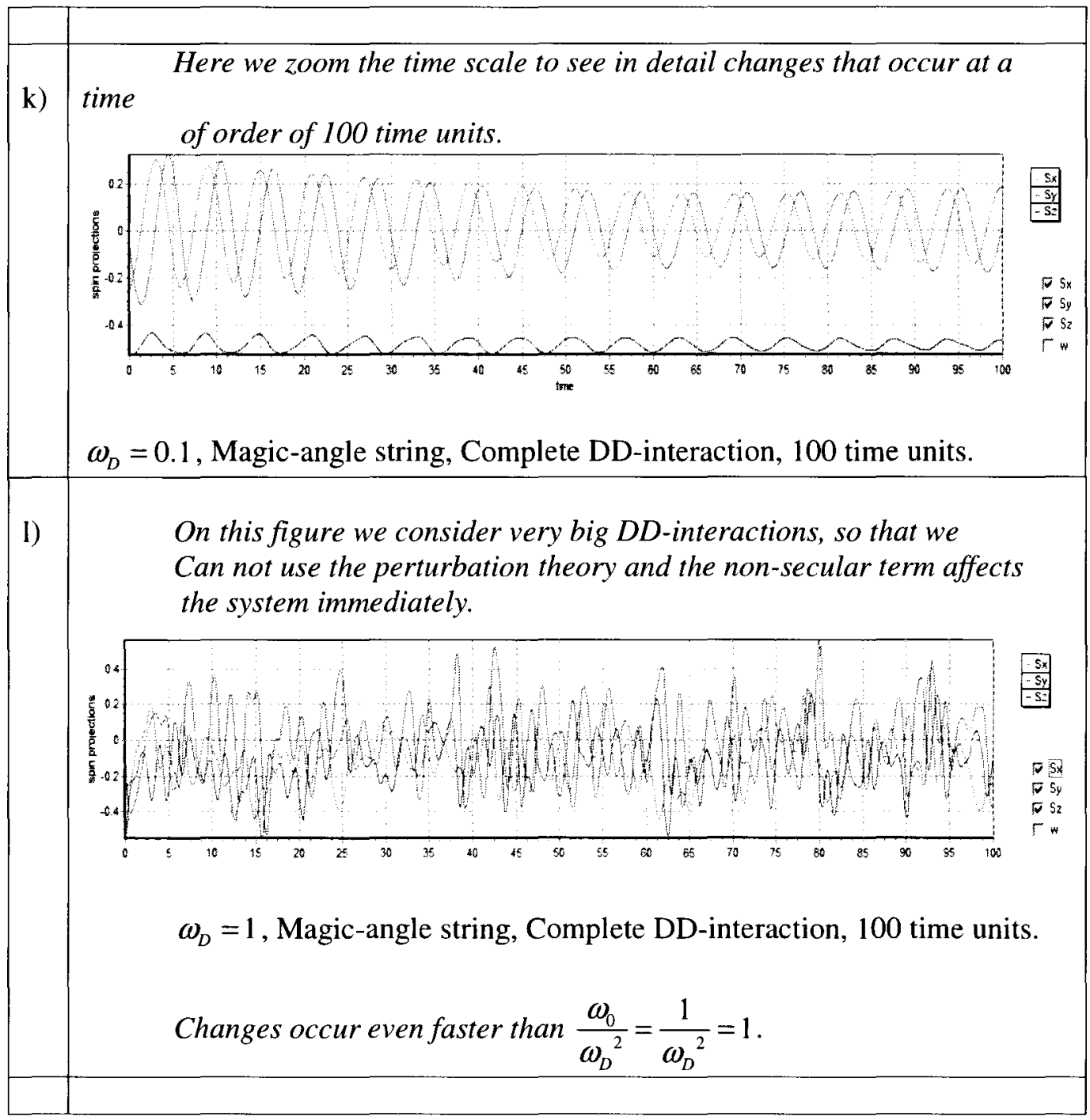

Figure 2.2.2 time evolution of components of the total spin in cases of different alignments, types and strengths of dipolar interaction

Interestingly enough, for the model of classical spins the non-secular terms bringa small contribution as predicted by quantum theory, whereas classically they have the same order of magnitude as Secular terms. Thus, naively we could expect non-secular terms to act already at times of $\frac{1}{\omega_{D}}$, but due to the correspondence principle they act only at times of order of $\frac{\omega_{0}}{\omega_{D}{ }^{2}}$ as predicted by quantum theory. This example demonstrates how we can apply the classical model to get results which are in agreement with quantum theory. In other words, the correspondence principle allows us to use quantum theory to predict the behaviour of a classical model and vice versa. Thus, provided the correspondence principle for some particular observable, we can we can use whatever approach is comfortable (Heisenberg's, Schrodinger's, or classical) for studying the dynamics of this observable. 
However, figures $(\mathrm{j})$ and $(\mathrm{k})$ show that non-secular terms still bring a contribution of the order of $\left(\frac{\omega_{D}}{\omega_{0}}\right)$ to $\mathrm{X}, \mathrm{Y}$ and $\mathrm{Z}$-components of the total spin. Thus, even though perturbation theory predicts that non-secular terms bring a second order contribution (i.e. $\left(\frac{\omega_{D}{ }^{2}}{\omega_{0}}\right)$ ) to the total energy of the system, it does not mean that the contribution of non-secular terms to other observables (e.g. individual components of the total spin) will be just as small - in fact, it can be even a contribution of the first $\operatorname{order}\left(\frac{\omega_{D}}{\omega_{0}}\right)$ 


\section{MODELLING}

\section{Basic System of Equations}

Lets consider the magnetodynamics of the assembly of magnetic moments with the classical equations of motion. For the $k$-th particle, i.e., for the magnetic moment $\vec{\mu}_{k}$ we have

$$
\frac{d \vec{\mu}_{k}}{d t}=\gamma_{k}\left(\vec{\mu}_{k} \times \vec{H}_{k}\right)
$$

here $\gamma_{s}$ is the gyromagnetic ratio for electrons. The field $\vec{H}_{k}$ in Eq. (3.1.1) is a full magnetic field affecting the $k$-th particle and in the considered case it comprises of:

1) external constant field $\vec{B} \square O z$;

2) uniaxial anisotropy field,

$$
\vec{H}_{A}=\left(H_{A} / \mu\right)\left(\vec{\mu}_{k} \vec{n}\right) \vec{n}, \quad H_{A}=2 E_{A} / \mu
$$

where $\vec{n}$ is a unit vector of the selected axis and $E_{A}$ the particle anisotropy energy;

3) feedback field $\vec{H}=(H, 0,0)$, generated by the current induced in the coil whose axis is directed along $O x$;

4) dipolar magnetic field $\vec{H}_{d d(k)}$ induced by the dipole-dipole pair interaction.

Directing the field $\vec{B}$, i.e., $O z$, along the particle selected axis, one has $\vec{n}=(0,0,1)$ so that the components of the effective field take the form

$$
\vec{H}=\left(H+H_{d x}, H_{d y}, B+\mu_{z} H_{A} / \mu+H_{d z}\right) .
$$

The local dipolar magnetic field $\vec{H}_{d d(k)}=-\partial U_{d d} / \partial \vec{\mu}_{k}$ at the location of the $k$ th particle is defined from the energy of the pairwise dipole-dipole interaction potential as 


$$
U_{d d}=\sum_{\substack{k, m \\ k>m}}^{N}\left[\frac{1}{r_{k m}^{3}}\left(\vec{\mu}_{k} \vec{\mu}_{m}\right)-\frac{3}{r_{k m}^{5}}\left(\vec{\mu}_{k} \vec{r}_{k m}\right)\left(\vec{\mu}_{m} \vec{r}_{k m}\right)\right]
$$

where $\vec{r}_{k m}$ is the radius-vector connecting the particle with the numbers $k$ and $m$.

We substitute Eq. (3.1.3) in Eq. (3.1.1) and, introducing a dimensionless time

$$
\tilde{t}=\omega_{0} t
$$

define the reference frequencies related to the external field $\vec{B}$ (Larmor frequency) and those related to the feedback, dipolar and anisotropy fields, respectively:

$$
\omega_{0}=\left|\gamma_{s}\right| B, \omega_{H}=\left|\gamma_{s}\right| B, \quad \omega_{D}=\left|\gamma_{s}\right| \mu / a^{3}, \omega_{A}=\left|\gamma_{s}\right| H_{A}
$$

Scaling the latter with the Larmor value, one gets a set of dimensionless parameters

$$
p_{A}=\frac{\omega_{A}}{\omega_{0}}=\frac{H_{A}}{B}, \quad p_{H}=\frac{\omega_{H}}{\omega_{0}}=\frac{H}{B}, \quad p_{D}=\frac{\omega_{D}}{\omega_{0}}=\frac{\mu}{a^{3} B},
$$

where $a$ is a mean interparticle distance. Since Eq. (3.1.1) conserves the modulus of the magnetic moment vector, for programming purposes it is convenient to use for the latter a unit vector $\vec{e}_{k}=\vec{\mu}_{k} / \mu$. However, for this paper we will keep using spin vectors instead of unitary ones.

In result, we get Eq. (3.1.1) in the form

$$
\begin{aligned}
& \dot{S}_{k}^{x}=-\left(1+p_{A} S_{k}^{z}\right) S_{k}^{y}-p_{D}\left(S_{k}^{y} \tilde{H}_{d z}^{(k)}-S_{k}^{z} \tilde{H}_{d y}^{(k)}\right), \\
& \dot{S}_{k}^{y}=\left(1+p_{A} S_{k}^{z}\right) S_{k}^{x}-p_{H} S_{k}^{z}-p_{D}\left(S_{k}^{z} \tilde{H}_{d x}^{(k)}-S_{k}^{x} \tilde{H}_{d z}^{(k)}\right), \\
& \dot{S}_{k}^{z}=p_{H} S_{y}^{x}-p_{D}\left(S_{k}^{x} \tilde{H}_{d y}^{(k)}-S_{k}^{y} \tilde{H}_{d x}^{(k)}\right)
\end{aligned}
$$

The dimensionless dipolar field at the $k$-th site is

$$
\vec{H}_{d d(k)} / B=p_{D} \overrightarrow{\tilde{H}}_{d d(k)} \quad, \quad \overrightarrow{\tilde{H}}_{d d(k)}=\sum_{\substack{m=1 \\ m \neq k}}^{N}\left[\frac{3}{\tilde{r}_{k m}^{5}} \vec{r}_{k m}\left(\vec{S}_{m} \vec{r}_{k m}\right)-\frac{1}{\tilde{r}_{k m}^{3}} \vec{S}_{m}\right],
$$

where $\overrightarrow{\tilde{r}}_{k m}=\vec{r}_{k m} / a$ are dimensionless vectors of interparticle distances. 


\section{Feedback Field Equations}

As mentioned, $O X$ axis of the coordinate framework is directed along the induction coil of the $L C R$ circuit. Thence, the non-stationary electromotive force and, accordingly, the electric current $I$ in the circuit are due to the $x$-component of the net magnetic moment of the system. The corresponding Kirchhoff equation is

$$
L \frac{d I}{d t}+R I+\frac{1}{C} \int_{0}^{t} I\left(t^{\prime}\right) d t^{\prime}=-\frac{d \Phi}{d t} \quad, \quad \Phi=\left(4 \pi / c_{0}\right) n \eta A m_{x},
$$

where $\Phi$ is the magnetic flux in a coil with $n$ turns and cross-section area $A$, the constant $c_{0}$ is the speed of light in vacuum, $\eta=V / V_{c}$ is the coil filling factor, $V$

is the volume of the sample containing ferromagnetic particles, and $V_{c}$ the inner volume of the coil. The quantity

$$
m_{x}=(\mu / V) \sum_{k} \frac{\vec{\mu}_{k}}{\mu_{k}}
$$

is the projection of the magnetization of the sample placed inside the coil on the axis of the latter.

We can introduce the unit vector $\vec{e}^{(k)}=\vec{\mu}_{k} / \mu$.

The induced current generates in the coil the magnetic (feedback) field

$$
H=\left(4 \pi n / c_{0} l\right) I
$$

The self-induction coefficient of a coil of length $l$ equals $L=4 \pi n^{2} A / l c_{0}^{2}$. Performing differentiation of Eq. (3.2.1) over time, using the time scale $\tilde{t}$ and the variable $p_{H}$, one arrives at

$$
\frac{d^{2}}{d \tilde{t}^{2}} p_{H}+2 \frac{\gamma}{\omega_{0}} \frac{d}{d \tilde{t}} p_{H}+\left(\frac{\omega_{r}}{\omega_{0}}\right)^{2} p_{H}=-4 \pi \beta\left(\frac{1}{N} \frac{d^{2}}{d \tilde{t}^{2}} \sum_{l=1}^{N} e_{x}^{(l)}\right)
$$

The coefficients at the left-hand side of Eq. (3.2.4) are expressed through the $L C R$ circuit parameters as $2 \gamma=c_{0}^{2} R / L=\omega_{r} / Q, \omega_{r}=c_{0} / \sqrt{L C}$; here $Q$ is the quality factor. The function in parentheses in the right-hand side of Eq. (3.2.4) is the averaged second derivative of the $x$-projection of the unit vector of magnetization. The combination of parameters

$$
\beta=\eta N \mu /\left(V H_{0}\right)
$$


is a coefficient that determines the intensity of magnetic coupling between the particle assembly and the coil. Using the estimate $a \approx(V / N)^{1 / 3}$ and the last of definitions (3.1.7), parameter $\beta$ may be presented in the form $\eta p_{D}$.

The set of $3 N$ equations (3.1.8) together with Eq. (3.1.4) is solved numerically for a sample containing $N$ particles arranged in various possible shapes.

Let us now figure out the initial conditions for the coil equation.

The equation for the resonator in integral form is:

$$
\frac{d}{d \tilde{t}} p_{H}+2 \frac{\gamma_{r}}{\omega_{0}} p_{H}+\left(\frac{\omega_{r}}{\omega_{0}}\right)^{2} \int_{0}^{i} p_{H} d \tilde{t}^{\prime}=-4 \pi \beta\left(\frac{1}{N} \frac{d}{d \tilde{t}} \sum_{l=1}^{N} e_{x}^{(l)}\right)
$$

Here

$$
\int_{0}^{i} p_{H} d \tilde{t}^{\prime} \square \int_{0}^{\bar{t}} I d \tilde{t}^{\prime}=\int_{0}^{\bar{t}} \dot{Q} d \tilde{t}^{\prime} \square \int_{0}^{\bar{t}} C \dot{U}_{C} d \tilde{t}^{\prime} \square U_{C},
$$

and $I$ is the current through the circuit, $C$ the capacitance and $U_{C}$ the voltage on the capacitor.

Of course, initially there is no current in the circuit. Thus:

$$
t=0: p_{H} \sqcup I=0 \text {, }
$$

and there is no initial voltage on the capacitor:

$$
t=0: U_{C}=0 \text {. }
$$

Substituting these conditions in (1), for $t=0$ we get:

$$
\begin{aligned}
& \frac{d}{d \tilde{t}} p_{H}+2 \frac{\gamma_{r}}{\omega_{0}} p_{H}+\left(\frac{\omega_{r}}{\omega_{0}}\right)^{2} \int_{0}^{\tilde{t}} p_{H} d \tilde{t}^{\prime}=-4 \pi \beta\left(\frac{1}{N} \frac{d}{d \tilde{t}} \sum_{l=1}^{N} e_{x}^{(l)}\right) ; \\
& \left.\frac{d}{d \tilde{t}} p_{H}\right|_{t=0}=-\left.4 \pi \beta\left(\frac{1}{N} \frac{d}{d \tilde{t}} \sum_{l=1}^{N} e_{x}^{(l)}\right)\right|_{t=0} .
\end{aligned}
$$

Finally, for the initial conditions we have:

$$
\begin{aligned}
& \left.p_{H}\right|_{t=0}=0, \\
& \left.\frac{d}{d \tilde{t}} p_{H}\right|_{t=0}=-\left.4 \pi \beta\left(\frac{1}{N} \frac{d}{d \tilde{t}} \sum_{l=1}^{N} e_{x}^{(l)}\right)\right|_{t=0}
\end{aligned}
$$




\section{SWITCHING TO THE ROTATING FRAME}

The correspondence principle, proved in part I, allows us, using the classical approach we can switch to the rotating frame (RF) to get rid of big terms. Since, because of the field induced due to the coil we still cannot get rid of high-frequency terms, it does not matter what form of the Hamiltonian to use (complete or truncated - they both are symmetric and the correspondence principle works for them).

Due to equivalence of quantum and classical equations, it is more comfortable to use a classical system. Let us write down this system (without taking into account anisotropy) and transfer it to RF.

\section{Equations for the Induced Field of the Coil in the Rotating Frame}

Since the dipole interactions in the studied situations are very weak, the corresponding terms in equations (3.1.8), as well as the terms with the feedback field for small value of parameter $\beta$, are much smaller than the contribution of the major terms on the right side of the two first equations (these terms are due to Zeeman interactions in the static field $B$ ). The standard approach in such a situation is switching to the rotating frame to get rid of big Zeeman terms. To do this, the oscillating external magnetic field should be split into clockwise and counterclockwise components and then the last one is neglected. The dipole Hamiltonian in this approach is reduced to its secular part. Unfortunately, such an approach for the field described by equation (3.2.4) leads to appearance of big terms in this equation in the rotating frame, which happens because of time derivatives in equation (3.2.4). Lets show it with more details.

Transformation of 3 -dimensional vectors $(X, Y, Z)$ to a rotating frame is performed in the following way:

$$
\begin{aligned}
& \left(\begin{array}{l}
X \\
Y \\
Z
\end{array}\right)=\left(\begin{array}{ccc}
\cos \omega t & -\sin \omega t & 0 \\
\sin \omega t & \cos \omega t & 0 \\
0 & 0 & 1
\end{array}\right)\left(\begin{array}{l}
X^{\prime} \\
Y^{\prime} \\
Z^{\prime}
\end{array}\right) \\
& \text { Here "prime signs" indicate components in rotating frame. } \\
& \text { For simplicity let us introduce the notations } \\
& \cos \omega t \equiv c \\
& \sin \omega t \equiv s
\end{aligned}
$$


Symbol $\omega$ means the frequency of our rotating frame.

Lets try to split the resonator field into clockwise and counterclockwise components as it is done for harmonic external field (see figure below).

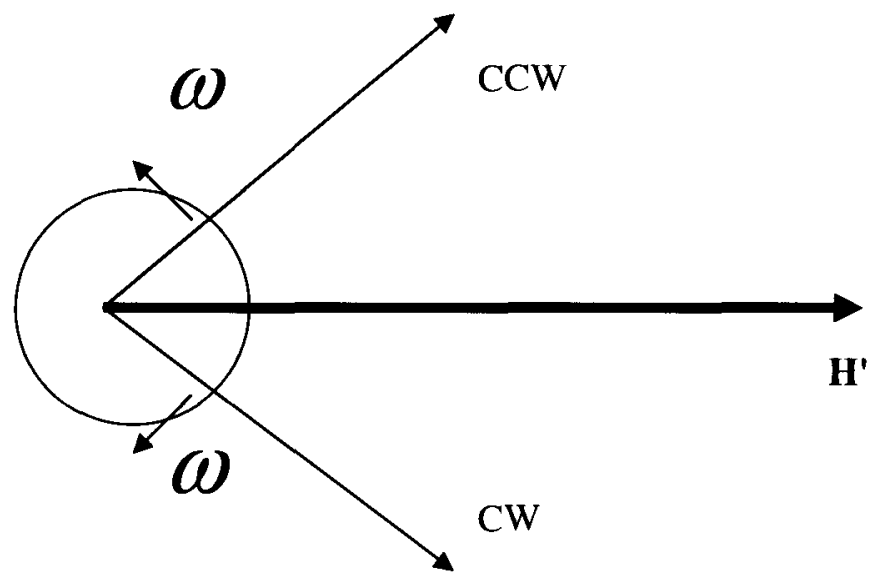

Figure 4.1.1 decomposition of the field of the resonator into $\mathrm{CW}$ and $\mathrm{CCW}$ components.

The CCW-component will bring a negligible contribution to motion of spins [2]. Also, the CCW-can be easily obtained from the $\mathrm{CW}$-component. Thus, we need only take into account the $\mathrm{CW}$-component. Since X-projections of CW and CCWcomponents are equal, the field of the coil can be represented as the doubled $\mathrm{X}$ projection of $\mathrm{CW}$-component:

$$
p_{H}^{\prime}=2\left(c h_{x}-s h_{y}\right)
$$

Here $h_{x}^{\prime}, h_{y}^{\prime}$ are slow functions of time representing the clockwise component of the resonator field in the rotating frame. $m \equiv \beta \frac{1}{N} \sum_{l=1}^{N} e_{x}^{(l)}$ should be also transformed to the rotating frame:

$$
\left(\begin{array}{c}
m \\
0 \\
0
\end{array}\right)=\left(\begin{array}{ccc}
\cos \omega t & -\sin \omega t & 0 \\
\sin \omega t & \cos \omega t & 0 \\
0 & 0 & 1
\end{array}\right)\left(\begin{array}{c}
m_{x}^{\prime} \\
m_{y}^{\prime} \\
0
\end{array}\right) .
$$

Since our coil is along OX-axis, then the field created by the total spin in the coil is

$$
m_{x}^{\prime}=c m_{x}-s m_{y}
$$

Let us consider the Kirchhoff's equation for the coil .

$$
\frac{d^{2} p_{H}^{\prime}}{d t^{2}}+2 \gamma \frac{d p_{H}^{\prime}}{d t}+\omega_{r}^{2} p_{H}^{\prime}=-4 \pi \frac{d^{2} m_{x}^{\prime}}{d t^{2}}
$$


It can be easily seen that $p_{H}^{\prime}$ and $m_{x}^{\prime}$ have similar structure. Therefore, it is useful bring them together. Then, from equation (4.1.4) we get:

$$
\frac{d^{2}}{d t^{2}}\left(p_{H}^{\prime}+4 \pi m_{x}^{\prime}\right)+2 \gamma \frac{d p_{H}^{\prime}}{d t}+\omega_{r}^{2} p_{H}^{\prime}=0 \text {. }
$$

Using (4.1.3) and (4.1.2) and putting together terms with $s$ and $c$ we will get the following expression:

$$
\frac{d^{2}}{d t^{2}}\left(c\left[2 h_{x}+4 \pi m_{x}\right]-s\left[2 h_{y}+4 \pi m_{y}\right]\right)+2 \gamma \frac{d p_{H}^{\prime}}{d t}+\omega_{r}^{2} p_{H}^{\prime}=0 .
$$

For simplicity we can also introduce notations:

$$
\begin{aligned}
2 h_{x}+4 \pi m_{x} & \equiv f_{x} \\
2 h_{y}+4 \pi m_{y} & \equiv f_{y}
\end{aligned}
$$

Now, let us modify the second term from (4.1.5):

$$
\frac{d p_{H}^{\prime}}{d t}=2 \frac{d}{d t}\left(c h_{x}-s h_{x}\right)=2\left(c\left(\dot{h}_{x}-\omega h_{y}\right)-s\left(\dot{h}_{y}+\omega h_{x}\right)\right)
$$

Performing similar transformations for the first term in (4) we get:

$$
\begin{aligned}
& \frac{d^{2}}{d t^{2}}\left(p_{H}^{\prime}+4 \pi m_{x}^{\prime}\right)=\frac{d^{2}}{d t^{2}}\left(c f_{x}-s f_{y}\right)=\frac{d}{d t}\left(c\left(\dot{f}_{x}-\omega f_{y}\right)-s\left(\dot{f}_{y}+\omega f_{x}\right)\right)= \\
& =c\left(\ddot{f}_{x}-2 \omega \dot{f}_{y}-\omega^{2} f_{x}\right)-s\left(\ddot{f}_{y}+2 \omega \dot{f}_{x}-\omega^{2} f_{y}\right)
\end{aligned}
$$

Substituting (4.1.7), (4.1.8) in (4.1.5) and bringing together terms with «+c» and «-s» we get a system of equations:

$$
\begin{aligned}
& +c: \ddot{f}_{x}-2 \omega_{r} \dot{f}_{y}-\omega_{r}^{2} f_{x}+4 \gamma\left(\dot{h}_{x}-\omega h_{y}\right)+2 \omega^{2} h_{x}=0 \\
& -s: \ddot{f}_{y}+2 \omega_{r} \dot{f}_{x}-\omega_{r}^{2} f_{y}+4 \gamma\left(\dot{h}_{y}+\omega h_{x}\right)+2 \omega^{2} h_{y}=0
\end{aligned}
$$

Now we can substitute this into (4.1.6), and after simple transformations we arrive at:

$$
\begin{aligned}
& \ddot{h}_{x}-2 \omega \dot{h}_{y}-\omega^{2} h_{x}+2 \gamma\left(\dot{h}_{x}-\omega h_{y}\right)+\omega_{r}^{2} h_{x}=-2 \pi\left(\ddot{m}_{x}-2 \omega \dot{m}_{y}-\omega^{2} m_{x}\right) \\
& \ddot{h}_{y}+2 \omega \dot{h}_{x}-\omega^{2} h_{y}+2 \gamma\left(\dot{h}_{y}+\omega h_{x}\right)+\omega_{r}^{2} h_{y}=-2 \pi\left(\ddot{m}_{y}+2 \omega \dot{m}_{x}-\omega^{2} m_{y}\right)
\end{aligned} .
$$

Let us isolate $h_{x}$ in the first equation and $h_{y}$ in the second:

$$
\begin{aligned}
& \ddot{h}_{x}+2 \gamma \dot{h}_{x}+\left(\omega_{r}^{2}-\omega^{2}\right) h_{x}=-2 \pi\left(\ddot{m}_{x}-2 \omega \dot{m}_{y}-\omega^{2} m_{x}\right)+2 \omega\left(\dot{h}_{y}+\gamma h_{y}\right) \\
& \ddot{h}_{y}+2 \gamma \dot{h}_{y}+\left(\omega_{r}^{2}-\omega^{2}\right) h_{y}=-2 \pi\left(\ddot{m}_{y}+2 \omega \dot{m}_{x}-\omega^{2} m_{y}\right)+2 \omega\left(\dot{h}_{x}-\gamma h_{x}\right)
\end{aligned}
$$

These are equations for the resonator in a rotating frame.

Thus, the attempt to perform splitting and neglecting the counterclockwise term leads to the appearance of big terms (of the order of $\omega^{2} \approx \omega_{0}^{2}$ ) in the feedback equation when we switch to the rotating frame. This happens because of time derivatives in the equation (3.2.4). Therefore, the differential equation for the resonator should be solved in stationary frame, which does not give big terms, but keeps high frequency terms. Thus, since switching to rotating frame allows us to get 
rid of big terms, it still does not help us to get rid of high frequency terms. Also we see that there is no necessity to use the truncated dipole Hamiltonian - we can as well use a complete dipole Hamiltonian.

\section{Equations for Spins in the Rotating Frame}

We can get the initial system of equation either after commuting spinoperators with the Hamiltonian, or just writing the components of gyration equations.

$$
\begin{aligned}
& \dot{S}_{x}^{j}=-\omega_{0} S_{y}^{j}-\omega_{L} D_{x}^{j} \\
& \dot{S}_{y}^{j}=\omega_{0} S_{x}^{j}-\omega_{L} D_{y}^{j}-H S_{z}^{j} \\
& \dot{S}_{z}^{j}=H S_{y}^{j}-\omega_{L} D_{z}^{j} .
\end{aligned}
$$

Here we have used the notation $D_{x, y, z}^{j}$ which are contributions of dipolar interactions to the spin's time derivative, and $\omega_{L}$ are "weights" of those contributions, which define the strength of DD-interactions. which are nothing but $-\omega_{L} D_{x, y, z}^{j}=\left\{\gamma_{j}\left[\vec{S}_{j} \times \vec{H}_{j}\right]\right\}_{x, y, z}$ and $H_{i}^{\alpha}=-\partial E_{d d} / \partial \mu_{l}^{\alpha}=-\sum_{k \neq l} D_{l k}^{\alpha \beta} \mu_{k}^{\beta} . H$ is the field of the coil in X-direction which includes both the field generated by an external source and the induced field.

Let us rewrite this system in a more convenient matrix form:

$$
\left(\begin{array}{c}
\dot{S}_{j}^{x} \\
\dot{S}_{j}^{y} \\
\dot{S}_{j}^{z}
\end{array}\right)=\left[\left(\begin{array}{ccc}
0 & -\omega_{0} & 0 \\
\omega_{0} & 0 & 0 \\
0 & 0 & 0
\end{array}\right)+\left(\begin{array}{ccc}
0 & 0 & 0 \\
0 & 0 & -H \\
0 & H & 0
\end{array}\right)\right]\left(\begin{array}{c}
S_{j}^{x} \\
S_{j}^{y} \\
S_{j}^{z}
\end{array}\right)-\omega_{L}\left(\begin{array}{c}
D_{j}^{x} \\
D_{j}^{y} \\
D_{j}^{z}
\end{array}\right) \quad(4.2 .2)
$$

Then make a substitution corresponding to switching to RF:

$$
\left(\begin{array}{l}
S_{j}^{x} \\
S_{j}^{y} \\
S_{j}^{z}
\end{array}\right) \rightarrow\left(\begin{array}{ccc}
\cos \omega t & -\sin \omega t & 0 \\
\sin \omega t & \cos \omega t & 0 \\
0 & 0 & 1
\end{array}\right)\left(\begin{array}{l}
S_{j}^{x} \\
S_{j}^{y} \\
S_{j}^{z}
\end{array}\right)=\left(\begin{array}{c}
S_{j}^{x} \cos \omega t-S_{j}^{y} \sin \omega t \\
S_{j}^{x} \sin \omega t+S_{j}^{y} \cos \omega t \\
S_{j}^{z}
\end{array}\right) \equiv\left(\begin{array}{l}
S_{j}^{\prime x} \\
S_{j}^{\prime y} \\
S_{j}^{\prime z}
\end{array}\right)
$$

Thus, for time derivatives we will have:

$$
\left(\begin{array}{c}
\dot{S}_{j}^{x} \\
\dot{S}_{j}^{y} \\
\dot{S}_{j}^{z}
\end{array}\right) \rightarrow \omega\left(\begin{array}{ccc}
-\sin \omega t & -\cos \omega t & 0 \\
\cos \omega t & -\sin \omega t & 0 \\
0 & 0 & 1
\end{array}\right)\left(\begin{array}{l}
S_{j}^{x} \\
S_{j}^{y} \\
S_{j}^{z}
\end{array}\right)+\left(\begin{array}{ccc}
\cos \omega t & -\sin \omega t & 0 \\
\sin \omega t & \cos \omega t & 0 \\
0 & 0 & 1
\end{array}\right)\left(\begin{array}{c}
\dot{S}_{j}^{x} \\
\dot{S}_{j}^{y} \\
\dot{S}_{j}^{z}
\end{array}\right)
$$

Further, all the values in the rotating frame will be written without a prime symbol and values in the lab frame will be written with prime. For instance, symbols $D_{j}^{\prime x}, D_{j}^{\prime y}, D_{j}^{\prime z}$ will mean contributions of dipole terms, which depend on spins in the stationary (lab) frame. In the same manner the field of the coil $H^{\prime}$ will depend on $\vec{S}^{\prime j}$. I.e. those values will be calculated according to "old" (written for the lab frame) equations. But instead of $\vec{S}^{j}$ we will now substitute $\vec{S}^{j}$ into them. 
Thus, for the new variables we get:

$$
\begin{aligned}
& \left(\begin{array}{l}
\dot{S}_{j}^{x} \\
\dot{S}_{j}^{y} \\
\dot{S}_{j}^{z}
\end{array}\right) \rightarrow \omega\left(\begin{array}{ccc}
-\sin \omega t & -\cos \omega t & 0 \\
\cos \omega t & -\sin \omega t & 0 \\
0 & 0 & 1
\end{array}\right)\left(\begin{array}{l}
S_{j}^{x} \\
S_{j}^{y} \\
S_{j}^{z}
\end{array}\right)+\left(\begin{array}{ccc}
\cos \omega t & -\sin \omega t & 0 \\
\sin \omega t & \cos \omega t & 0 \\
0 & 0 & 1
\end{array}\right)\left(\begin{array}{l}
\dot{S}_{j}^{x} \\
\dot{S}_{j}^{y} \\
\dot{S}_{j}^{z}
\end{array}\right)= \\
& =\left[\left(\begin{array}{ccc}
0 & -\omega_{0} & 0 \\
\omega_{0} & 0 & 0 \\
0 & 0 & 0
\end{array}\right)+\left(\begin{array}{ccc}
0 & 0 & 0 \\
0 & 0 & -H^{\prime} \\
0 & H^{\prime} & 0
\end{array}\right)\right]\left(\begin{array}{c}
S_{j}^{\prime x} \\
S_{j}^{\prime y} \\
S_{j}^{\prime z}
\end{array}\right)-\omega_{L}\left(\begin{array}{c}
D_{j}^{\prime x} \\
D_{j}^{\prime y} \\
D_{j}^{\prime z}
\end{array}\right)
\end{aligned}
$$

Now, in order to isolate $\left(\begin{array}{c}\dot{S}_{j}^{x} \\ \dot{S}_{j}^{y} \\ \dot{S}_{j}^{z}\end{array}\right)$ we should multiply this equation from the left by the matrix of inverse transformation:

$$
\left(\begin{array}{ccc}
\cos \omega t & \sin \omega t & 0 \\
-\sin \omega t & \cos \omega t & 0 \\
0 & 0 & 1
\end{array}\right) \times
$$

Then, we get:

$$
\begin{aligned}
& \left(\begin{array}{ccc}
0 & -\omega & 0 \\
\omega & 0 & 0 \\
0 & 0 & 0
\end{array}\right)\left(\begin{array}{l}
S_{j}^{x} \\
S_{j}^{y} \\
S_{j}^{z}
\end{array}\right)+\left(\begin{array}{c}
\dot{S}_{j}^{x} \\
\dot{S}_{j}^{y} \\
\dot{S}_{j}^{z}
\end{array}\right)= \\
& =\left(\begin{array}{ccc}
\cos \omega t & \sin \omega t & 0 \\
-\sin \omega t & \cos \omega t & 0 \\
0 & 0 & 1
\end{array}\right)\left[\left(\begin{array}{ccc}
0 & -\omega_{0} & 0 \\
\omega_{0} & 0 & 0 \\
0 & 0 & 0
\end{array}\right)+\left(\begin{array}{ccc}
0 & 0 & 0 \\
0 & 0 & -H^{\prime} \\
0 & H^{\prime} & 0
\end{array}\right)\right]\left(\begin{array}{l}
S_{j}^{\prime x} \\
S_{j}^{\prime y} \\
S_{j}^{\prime z}
\end{array}\right)- \\
& -\omega_{L}\left(\begin{array}{ccc}
\cos \omega t & \sin \omega t & 0 \\
-\sin \omega t & \cos \omega t & 0 \\
0 & 0 & 1
\end{array}\right)\left(\begin{array}{c}
D_{j}^{\prime x} \\
D_{j}^{\prime y} \\
D_{j}^{\prime z}
\end{array}\right)
\end{aligned}
$$

Now, for simplicity we will introduce the notation:

$\cos \omega t \equiv c$

$\sin \omega t \equiv s$

Taking into account the equations for $\vec{S}_{j}^{\prime}$ and the fact that

$$
\left(\begin{array}{cc}
c & s \\
-s & c
\end{array}\right)\left(\begin{array}{cc}
0 & -\omega_{0} \\
\omega_{0} & 0
\end{array}\right)\left(\begin{array}{cc}
c & -s \\
s & c
\end{array}\right)=\omega_{0}\left(\begin{array}{cc}
c & s \\
-s & c
\end{array}\right)\left(\begin{array}{cc}
-s & -c \\
c & -s
\end{array}\right)=\omega_{0}\left(\begin{array}{cc}
0 & -1 \\
1 & 0
\end{array}\right),
$$




$$
\begin{aligned}
& \left(\begin{array}{ccc}
c & s & 0 \\
-s & c & 0 \\
0 & 0 & 1
\end{array}\right)\left(\begin{array}{ccc}
0 & 0 & 0 \\
0 & 0 & -H \\
0 & H & 0
\end{array}\right)=\left(\begin{array}{ccc}
0 & 0 & -H s \\
0 & 0 & -H c \\
0 & H & 0
\end{array}\right) \text { and } \\
& \left(\begin{array}{ccc}
c & s & 0 \\
-s & c & 0 \\
0 & 0 & 1
\end{array}\right)\left(\begin{array}{c}
D_{j}^{\prime x} \\
D_{j}^{\prime y} \\
D_{j}^{\prime z}
\end{array}\right)=\left(\begin{array}{c}
D_{j}^{\prime x} c+D_{j}^{\prime y} s \\
-D_{j}^{\prime x} s+D_{j}^{\prime \prime} c \\
D_{j}^{\prime z}
\end{array}\right) .
\end{aligned}
$$

We can rewrite the right-hand side of (4.2.4) in the following form:

$$
\begin{aligned}
& \left(\begin{array}{ccc}
0 & -\omega & 0 \\
\omega & 0 & 0 \\
0 & 0 & 0
\end{array}\right)\left(\begin{array}{l}
S_{j}^{x} \\
S_{j}^{y} \\
S_{j}^{z}
\end{array}\right)+\left(\begin{array}{c}
\dot{S}_{j}^{x} \\
\dot{S}_{j}^{y} \\
\dot{S}_{j}^{z}
\end{array}\right)=\left(\begin{array}{ccc}
0 & -\omega_{0} & 0 \\
\omega_{0} & 0 & 0 \\
0 & 0 & 0
\end{array}\right)\left(\begin{array}{l}
S_{j}^{x} \\
S_{j}^{y} \\
S_{j}^{z}
\end{array}\right)+ \\
& +\left(\begin{array}{ccc}
0 & 0 & -H^{\prime} S \\
0 & 0 & -H^{\prime} c \\
0 & H^{\prime} & 0
\end{array}\right)\left(\begin{array}{c}
S_{j}^{\prime x} \\
S_{j}^{\prime y} \\
S_{j}^{\prime z}
\end{array}\right)-\omega_{L}\left(\begin{array}{c}
D_{j}^{\prime x} c+D_{j}^{\prime y} s \\
-D_{j}^{\prime x} s+D_{j}^{\prime \prime} c \\
D_{j}^{\prime z}
\end{array}\right)
\end{aligned}
$$

If on the right-hand side we leave only time derivatives, we get:

$$
\left(\begin{array}{c}
\dot{S}_{j}^{x} \\
\dot{S}_{j}^{y} \\
\dot{S}_{j}^{z}
\end{array}\right)=\left(\omega_{0}-\omega\right)\left(\begin{array}{ccc}
0 & -1 & 0 \\
1 & 0 & 0 \\
0 & 0 & 0
\end{array}\right)\left(\begin{array}{c}
S_{j}^{x} \\
S_{j}^{y} \\
S_{j}^{z}
\end{array}\right)+\left(\begin{array}{ccc}
0 & 0 & -H^{\prime} s \\
0 & 0 & -H^{\prime} c \\
0 & H^{\prime} & 0
\end{array}\right)\left(\begin{array}{c}
S_{j}^{\prime x} \\
S_{j}^{\prime y} \\
S_{j}^{\prime z}
\end{array}\right)-\omega_{L}\left(\begin{array}{c}
D_{j}^{\prime x} c+D_{j}^{\prime y} s \\
-D_{j}^{\prime x} s+D_{j}^{\prime y} c \\
D_{j}^{\prime z}
\end{array}\right)
$$

Since $\omega_{0}$ can vary from particle to particle, it is reasonable to choose some average value of $\left\langle\omega_{0}\right\rangle$ as $\omega$. Then all the Zeeman terms will have an order of the deviation of each individual Zeeman frequency from $\left\langle\omega_{0}\right\rangle$.

If all the $\omega_{0}$ are similar $\left(\omega=\omega_{0}\right)$, then the Zeeman terms vanish and (4.2.5) turns into

$$
\left(\begin{array}{c}
\dot{S}_{j}^{x} \\
\dot{S}_{j}^{y} \\
\dot{S}_{j}^{z}
\end{array}\right)=\left(\begin{array}{ccc}
0 & 0 & -H^{\prime} s \\
0 & 0 & -H^{\prime} c \\
0 & H^{\prime} & 0
\end{array}\right)\left(\begin{array}{c}
S_{j}^{\prime x} \\
S_{j}^{\prime y} \\
S_{j}^{\prime z}
\end{array}\right)-\omega_{L}\left(\begin{array}{c}
D_{j}^{\prime x} c+D_{j}^{\prime y} s \\
-D_{j}^{\prime x} s+D_{j}^{\prime y} c \\
D_{j}^{\prime z}
\end{array}\right)
$$

Thus, after writing each component of equation (4.2.5) we get:

$\dot{S}_{j}^{x}=-\left(\omega_{0}^{j}-\omega\right) S_{j}^{y}-\left(H^{\prime} S_{j}^{z z}+\omega_{L} D_{j}^{\prime y}\right) \sin \omega t-\omega_{L} D_{j}^{\prime x} \cos \omega t$

$\dot{S}_{j}^{y}=\left(\omega_{0}^{j}-\omega\right) S_{j}^{x}-\left(H^{\prime} S_{j}^{z}+\omega_{L} D_{j}^{\prime y}\right) \cos \omega t+\omega_{L} D_{j}^{\prime x} \sin \omega t$

$\dot{S}_{j}^{z}=H^{\prime} S_{j}^{\prime y}-\omega_{L} D_{j}^{\prime z}$

where

$$
\begin{aligned}
& S_{j}^{\prime x}=S_{j}^{x} \cos \omega t-S_{j}^{y} \sin \omega t \\
& S_{j}^{\prime y}=S_{j}^{x} \sin \omega t+S_{j}^{y} \cos \omega t \\
& S_{j}^{\prime z}=S_{j}^{z}
\end{aligned}
$$


The index $j$ appears in $\omega_{0}^{j}$ because of the possible Zeeman frequency detuning.

For simulation purposes it is convenient to work in both frames (rotating and stationary) simultaneously. Terms $\tilde{H}_{d x}^{(k)}, \tilde{H}_{d v}^{(k)}, \tilde{H}_{d z}^{(k)}$ and $p_{H}$ are calculated in the stationary frame, whereas equations for $e_{x}^{(k)}, e_{y}^{\prime(k)}$ and $e_{z}^{\prime(k)}$ are solved in the rotating frame. Also, we will switch to dimensionless variables. Those equations become:

$$
\begin{aligned}
& \dot{S}_{k}^{\prime x}=-(1-\omega) S_{k}^{\prime y}-\left\{p_{H}^{\prime} S_{k}^{z}+p_{d} D_{y}^{\prime(k)}\right\} \sin \omega t-p_{d} D_{x}^{\prime(k)} \cos \omega t, \\
& \dot{S}_{k}^{\prime y}=(1-\omega) S_{x}^{\prime y}-\left\{p_{H}^{\prime} S_{k}^{z}+p_{d} D_{y}^{\prime(k)}\right\} \cos \omega t+p_{d} D_{x}^{\prime(k)} \sin \omega t, \\
& \dot{S}_{k}^{z}=p_{H} S_{k}^{y}-p_{d} D_{z}^{\prime(k)} .
\end{aligned}
$$

Here the following notations are used: $D_{x}^{(k)}=\left(e_{y}^{(k)} \tilde{H}_{d z}^{(k)}-e_{z}^{(k)} \tilde{H}_{d y}^{(k)}\right)$, $D_{y}^{(k)}=\left(e_{z}^{(k)} \tilde{H}_{d x}^{(k)}-e_{x}^{(k)} \tilde{H}_{d z}^{(k)}\right)$ and $D_{z}^{(k)}=\left(e_{x}^{(k)} \tilde{H}_{d y}^{(k)}-e_{y}^{(k)} \tilde{H}_{d x}^{(k)}\right)$. Similarly, in $\tilde{H}_{d x}^{(k)}$, $\tilde{H}_{d y}^{(k)}$ and $\tilde{H}_{d z}^{(k)}$, the values $D_{x}^{(k)}, D_{y}^{(k)}, D_{z}^{(k)}$ are calculated in the stationary frame.

Flowchart of the algorithm is represented on the following page. 


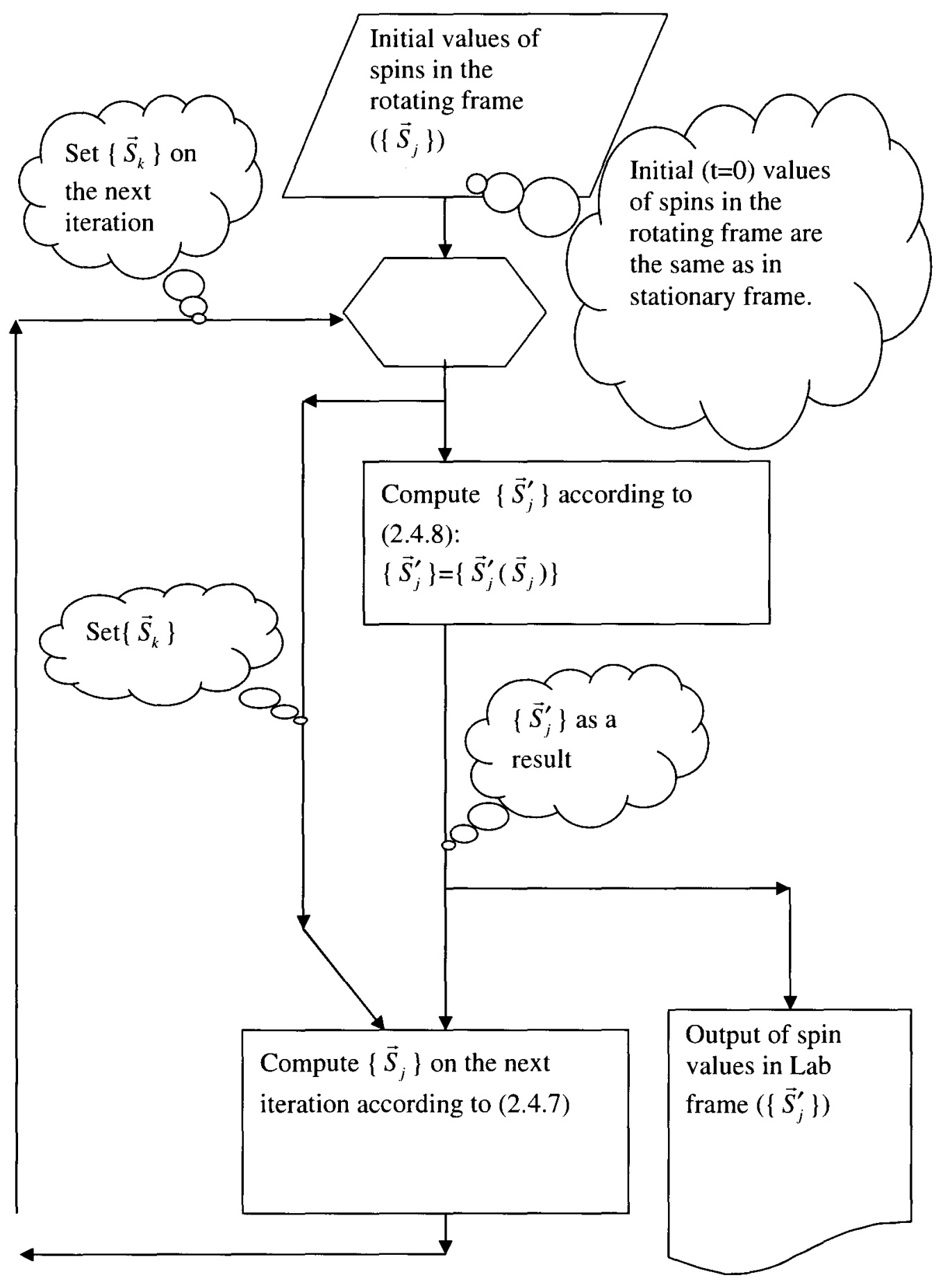

Figure 4.2.1 flowchart of the algorithm of simulation of spin dynamics in presence of the resonator in the rotating frame

The following set of figures demonstrates the role of dipolar and resonator terms in the Hamiltonian: 
These figures are generated in a rotating frame for 8 spins arranged in the shape of cube with initial total polarization $-50 \%$. With small dipolar interactions $\omega_{D}=10^{-5}$.

This graph depicts only the evolution of $\mathrm{X}, \mathrm{Y}$ and $\mathrm{Z}$ - components of the normalized total spin in presence of dipolar interactions. $\mathrm{X}$ and $\mathrm{Y}$-components slowly decay with time

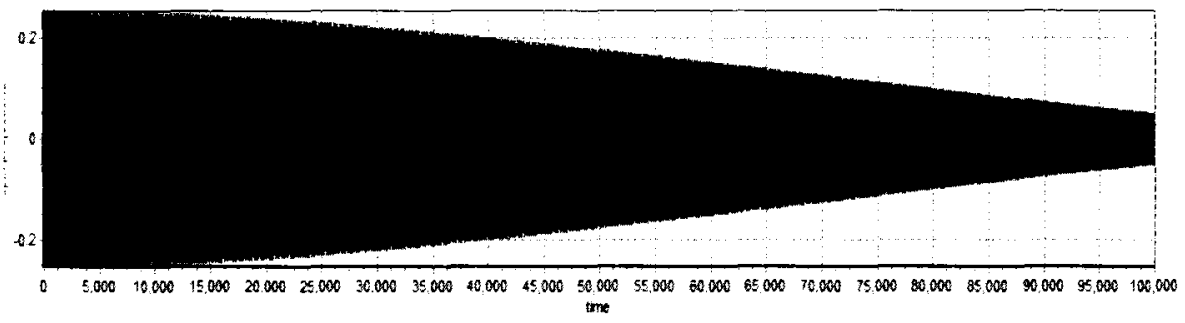

This graph depicts the behavior of the system taking into account the resonator, but without dipolar interactions $(\mathrm{X}, \mathrm{Y}$ and $\mathrm{Z}$ - components of the normalized total spin).

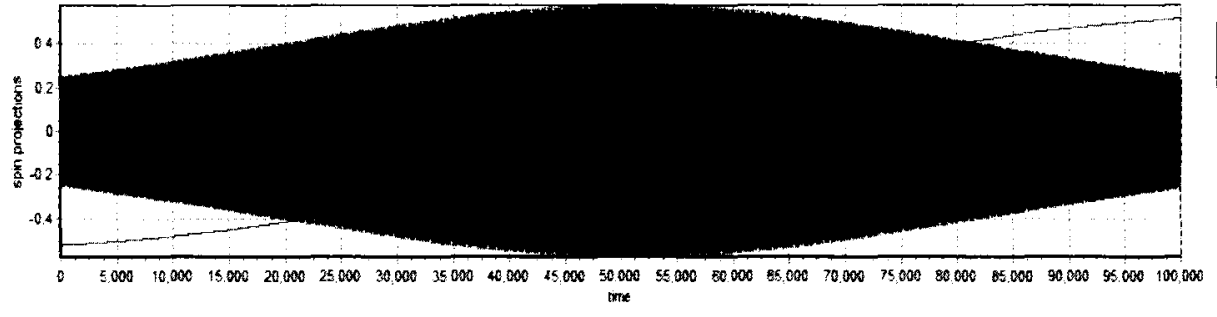

Interaction with the coil is $\beta=10^{-8}$ - so small that it creates an induced field of order $\omega_{D}=10^{-5}$. The decay coefficient is $\gamma=10^{-2}$. (the meanings of these two parameters are discussed in part III)

This is evolution of the induced field of the resonator.

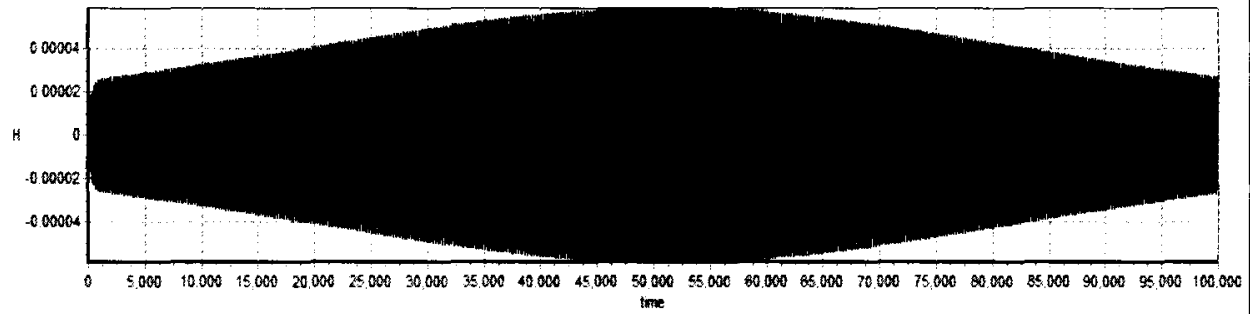

We can see that this kind of interaction acts at times of the same order as dipolar interaction.

The following two figures show the dynamics of spins and the induced resonator field respectively. 


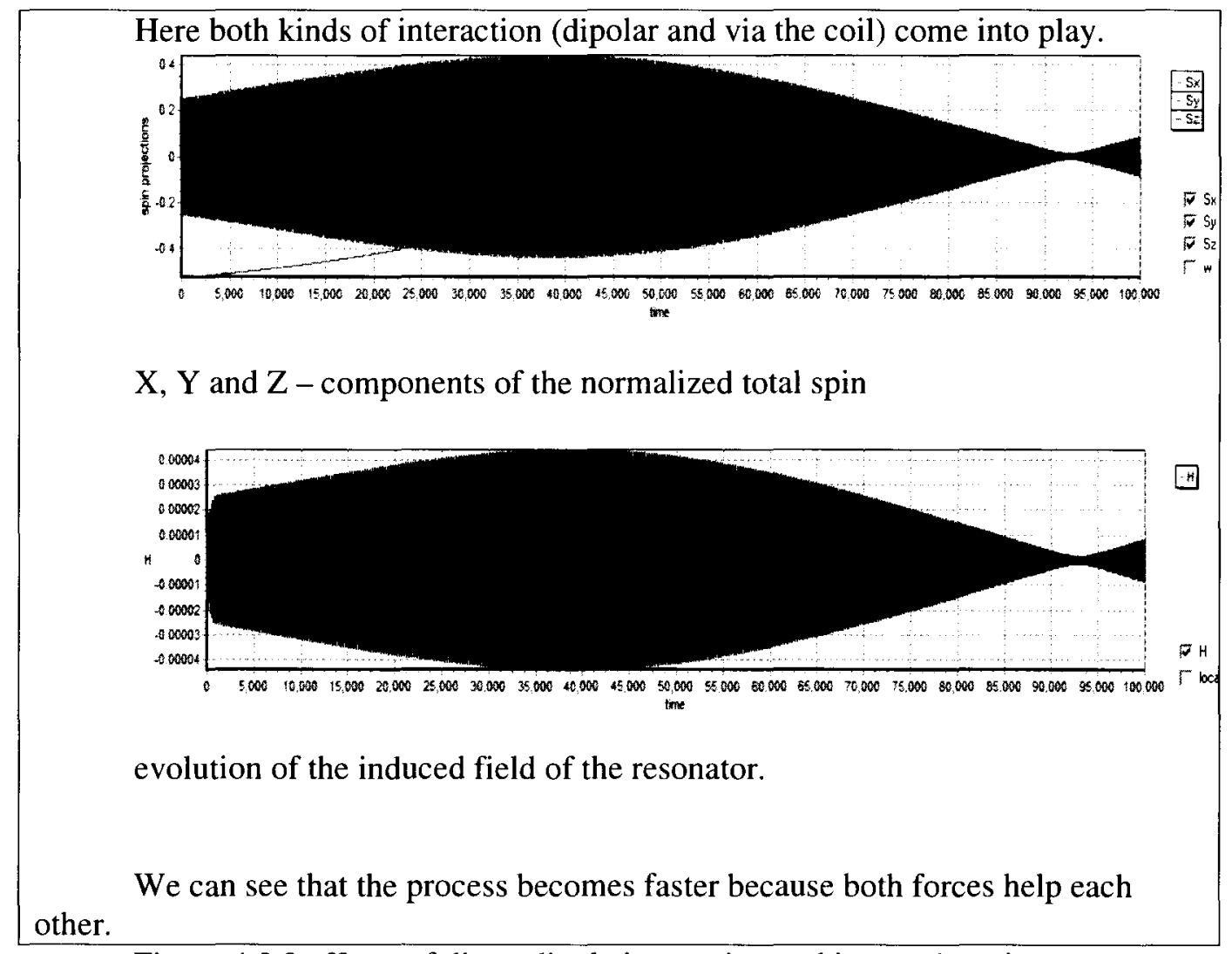

Figure 4.2.2 effects of direct dipole interaction and interaction via resonator

The figures above demonstrate that dipole interactions and interactions via the coil, when they act together, make the system evolve faster, than each of those interactions acting alone. Thus, the interaction between spins via a resonator can be treated as additional way of dipole interactions. 


\section{ANALYZING EQUATIONS FROM THE POINT OF VIEW OF THE CONTROL THEORY. INTERACTION OF A SPIN SYSTEM WITH THE RESONATOR AND USING THIS INTERACTION FOR CONTROL OF SPIN POLARIZATION.}

\section{Behavior of a Spin System Inside a Resonator}

Let's look at equations without DD-ineractions:

$$
\begin{aligned}
& \hat{H}=-B \hat{\mu}_{z}-\hat{H} \hat{\mu}_{x} \\
& \ddot{\hat{H}}+2 \gamma \dot{\hat{H}}+\omega^{2} \hat{H}=\beta \ddot{\hat{\mu}}_{x},
\end{aligned}
$$

Where $\beta=-4 \pi \frac{\rho}{N}$ and $\hat{\mu}$ is the total magnetic moment of the system. Here we have for simplicity redefined $\beta$ by including $4 \pi$ in it.

The solution of the differential equation for $\hat{H}$ is a sum of the General

Solution of Homogeneous Equation (GSHE) - $\hat{H}_{0}$ and the Particular Solution of Nonhomogenous Equation (PSNE) - $\hat{H}_{1}$.

PSNE can be written in terms of a transfer function:

After Laplace's transformation $\frac{\partial}{\partial t} \rightarrow s$ :

$\left(s^{2}+2 \gamma s+\omega^{2}\right) \hat{H}_{1}=\beta s^{2} \hat{\mu}_{x}$,

therefore,

$\hat{H}_{1}=\beta \frac{s^{2}}{s^{2}+2 \gamma_{s}+\omega^{2}} \hat{\mu}_{x} \equiv \beta f(s)\left\{\hat{\mu}_{x}(t)\right\} \equiv \beta f \hat{\mu}_{x}$. (now and later symbols like $f \hat{\mu}_{x}$ should be treated as one whole entity)

GSHE will look like

$\hat{H}_{0}=e^{-\gamma t}\left(\hat{A} \cos \omega^{\prime} t+\hat{B} \sin \omega^{\prime} t\right)$, where $\omega^{\prime}=\sqrt{\omega^{2}-\gamma^{2}}$.

Considering initial conditions:

$\hat{H}(t=0)=0$, 
$\dot{H}(t=0)=\beta \dot{\hat{\mu}}_{x}(t=0)$

We can find $\hat{A}$ and $\hat{B}$ :

$\left\{\begin{array}{l}0=\hat{H}(t=0)=\beta\left(\left.\left(f \hat{\mu}_{x}\right)\right|_{t=0}+\hat{A}\right) \\ \beta \dot{\hat{\mu}}_{x}(t=0)=\dot{\hat{H}}(t=0)=\beta\left(\left.\left(s f \hat{\mu}_{x}\right)\right|_{t=0}-\gamma \hat{A}+\omega^{\prime} \hat{B}\right)\end{array}\right.$

Thus,

$\left\{\begin{array}{l}\hat{A}=\left.\left(f \hat{\mu}_{x}\right)\right|_{t=0} \\ \hat{B}=\frac{\gamma}{\omega^{\prime}} \hat{A}-\left.\frac{1}{\omega^{\prime}}\left(s f \hat{\mu}_{x}\right)\right|_{t=0}=\left.\frac{1}{\omega^{\prime}}\left((\gamma-s) f \hat{\mu}_{x}+s \hat{\mu}_{x}\right)\right|_{t=0} .\end{array}\right.$

Finally,

$\hat{H}_{0}=e^{-r t}\left(\hat{A} \cos \omega^{\prime} t+\hat{B} \sin \omega^{\prime} t\right)=$

$=e^{-r}\left(\left.\left(f \hat{\mu}_{x}\right)\right|_{t=0} \cos \omega^{\prime} t+\left.\frac{1}{\omega^{\prime}}\left(((\gamma-s) f+s) \hat{\mu}_{x}\right)\right|_{t=0} \sin \omega^{\prime} t\right) \equiv \hat{G} e^{-r} \cos \left(\omega^{\prime} t+\hat{\phi}\right)$.

This GSHE is responsible for multiple flips.

Then, the expression for the total field will be:

$\hat{H}=\hat{H}_{0}+\hat{H}_{1}=\beta\left(f \hat{\mu}_{x}+\hat{G} e^{-n} \cos \left(\omega^{\prime} t+\phi\right)\right)$.

And the Hamiltonian:

$$
\hat{H}=-B \hat{\mu}_{z}(t)-\beta\left(f \hat{\mu}_{x}(t)+\hat{G} e^{-\gamma t} \cos \left(\omega^{\prime} t+\phi\right)\right) \hat{\mu}_{x}(t)
$$

An interesting situation occurs at big times

(5.1.4).

$t \sqsubset \frac{1}{\mu H}=\frac{1}{\mu \beta f \hat{\mu}_{x}(t)} \sqsubset \frac{1}{\gamma} \Leftrightarrow \mu \beta f \hat{\mu}_{x}(t) \sqsubset \gamma$. In this case GSHE decays already in the very beginning of the spin flip.

At these times, the Hamiltonian looks simply like

$$
\hat{H}=-B \hat{\mu}_{z}(t)-\beta \hat{\mu}_{x} f \hat{\mu}_{x}
$$

$(5.1 .5)$. 
Now let us study the asymptotic behavior of the system $(t \rightarrow \infty)$.

Due to the loss of energy through dissipation in the coil, the system will eventually come to the stationary state: $\dot{\hat{\mu}}_{z} \rightarrow 0$.

Since

$$
\dot{\hat{\mu}}_{z}=-i\left[\hat{\mu}_{z}, \hat{H}\right]=i\left[\hat{\mu}_{z}, \hat{\mu}_{x} \hat{H}\right]=i \hat{\mu}_{x}\left[\hat{\mu}_{z}, \hat{H}\right]+i\left[\hat{\mu}_{z}, \hat{\mu}_{x}\right] \hat{H}=i \hat{\mu}_{x}\left[\hat{\mu}_{z}, \hat{H}\right]-\gamma_{s} \hat{\mu}_{y} \hat{H}
$$

equals zero only if $\hat{H}(t)$ has a form which cannot cause any further flips. i.e. $\hat{H}(t)$ doesn't have harmonics of frequency close to $\omega_{0}$.

Thus, the asymptotic value of $\hat{H}$ has to satisfy

$$
\begin{aligned}
& \text { Fourier }\{\hat{H}(t \rightarrow \infty)\}\left(\Omega \approx \omega_{0}\right) \rightarrow 0 \\
& \text { Here Fourier }\{g(t)\}(\Omega)=\frac{1}{\pi} \int_{t_{11}}^{t \rightarrow \infty} g(t) e^{i \Omega t} d t \text { (we can count time from some }
\end{aligned}
$$
time $t_{0} \sqsubset \frac{1}{\gamma}-$ not to care about GSHE).

Thus,

$$
\hat{H}(t \rightarrow \infty)=\left.\left(f \hat{\mu}_{x}\right)\right|_{t \rightarrow \infty} \rightarrow 0 \Rightarrow \hat{\mu}_{x} \rightarrow 0 \Rightarrow \hat{\mu}_{z} \rightarrow \max \hat{\mu}_{z}
$$

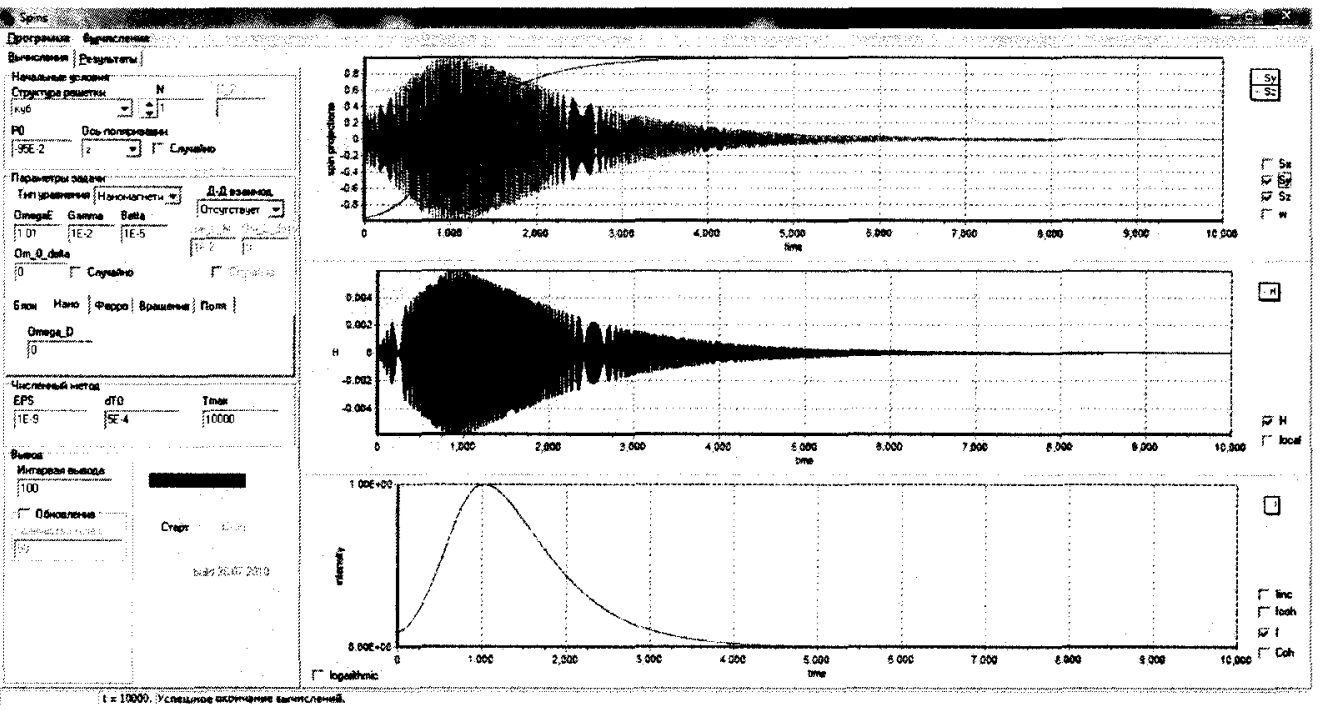

Figure 5.1.1 screenshot, demonstrating relaxation of a spin system via resistance of the resonator. Here we have high initial polarization, $\mu \beta f \hat{\mu}_{x}(t)=0.005, \gamma=0.01$ :

$\mu \beta f \hat{\mu}_{x}(t) \sqsubset \gamma$. As one can see, the Z-component of the total spin goes from polarized state to the state "spin up", which has the minimum energy. The $\mathrm{X}$ component of the total spin vanishes with time.

This figure (just as following figures) is a screenshot of the designed program. The top chart depicts the evolution of $\mathrm{Z}$ and $\mathrm{X}$ - normalized components of spin. The second chart from the top depicts the induced resonator field along with the external field. Bottom chart shows the intensity of radiation, produced by the spin sample. 


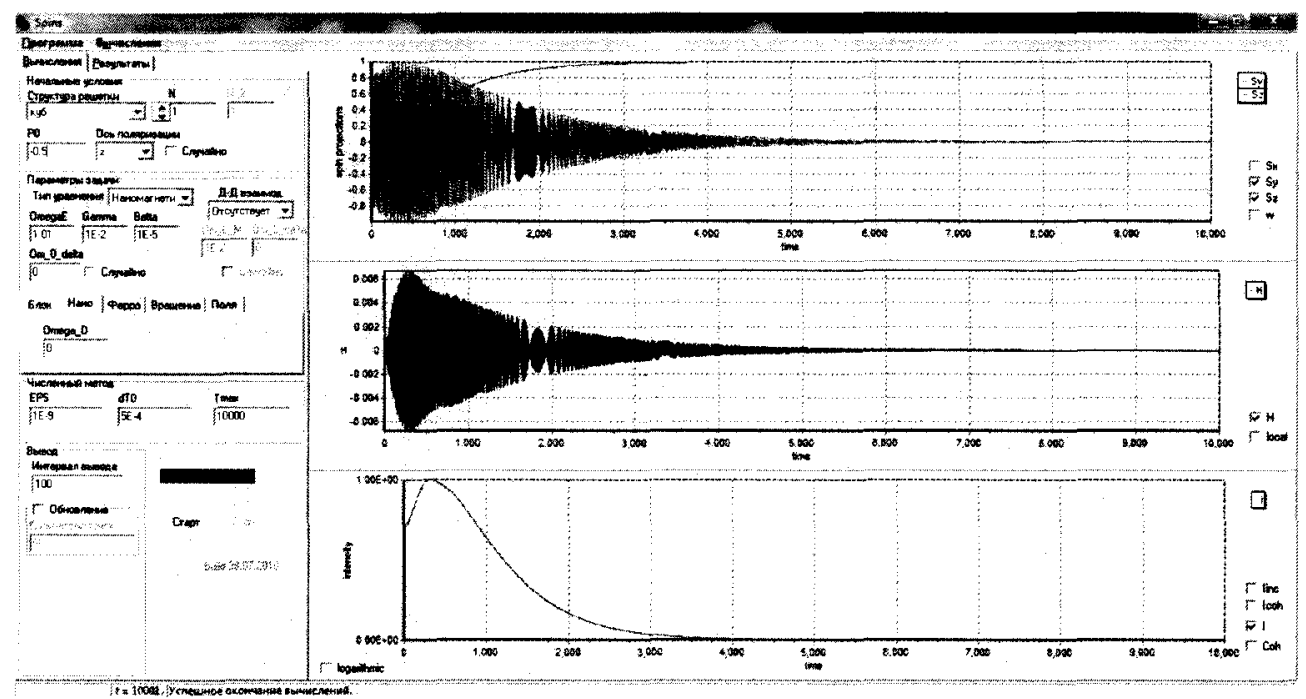

Figure 5.1.2 screenshot, demonstrating relaxation of a spin system via resistance of the resonator ( initial polarization is -0.5 )

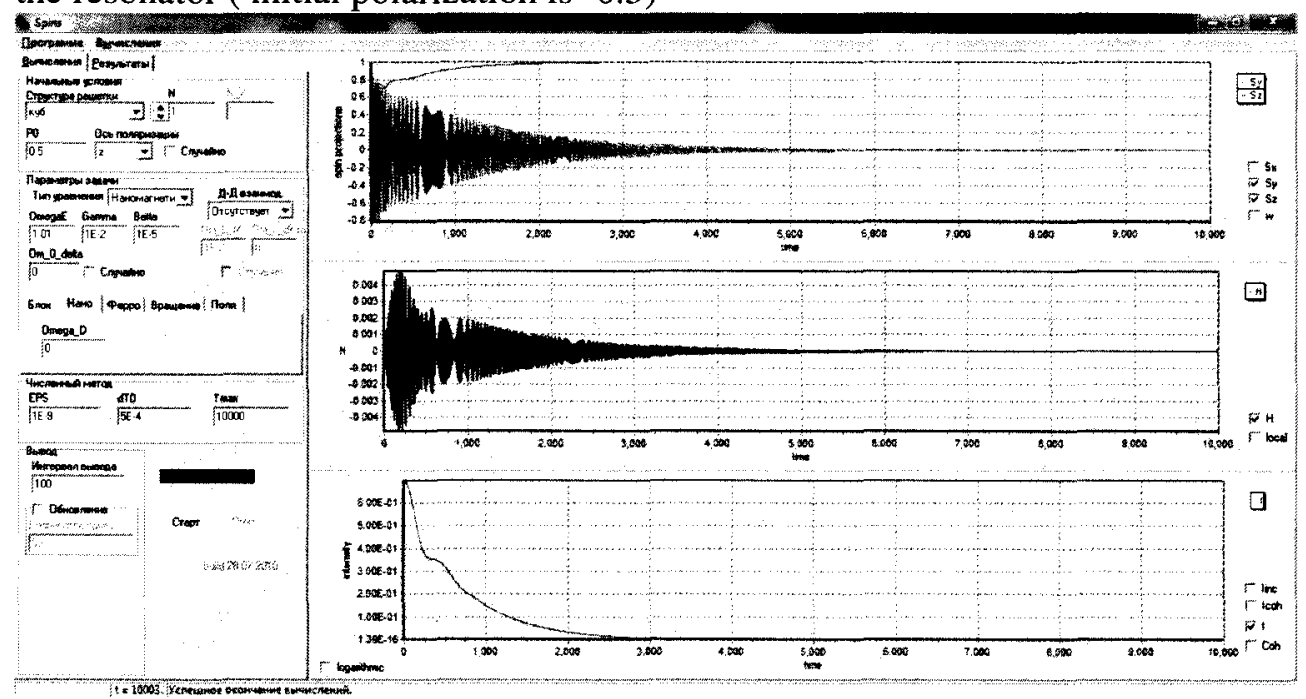

Figure 5.1.3 screenshot, demonstrating relaxation of a spin system via resistance of the resonator (initial polarization is +0.5 )

In figures (5.1.1 -5.1.3) one can see that regardless of initial polarization, final polarization approaches +1 , which is the state with minimum energy.

As predicted by (5.1.7), finally the spin is orientated entirely in Z-direction.

Speaking qualitatively, the PSNE affects on spin system in such a phase which always is trying to turn spins up. When the total polarization reaches it's maximum position, horizontal oscillations disappear. Since GSHE has already decayed, the coil already has no inertion. Therefore, when Spins align completely in the Z-direction, field of the coil disappears immediately and flips stop. 
It is interesting to observe what happens if we change the phase of $\beta f \hat{\mu}_{x}(t)$ (it can be done, for example, by changing the sign of $\beta \rightarrow-\beta$ ). In this case modeling shows:

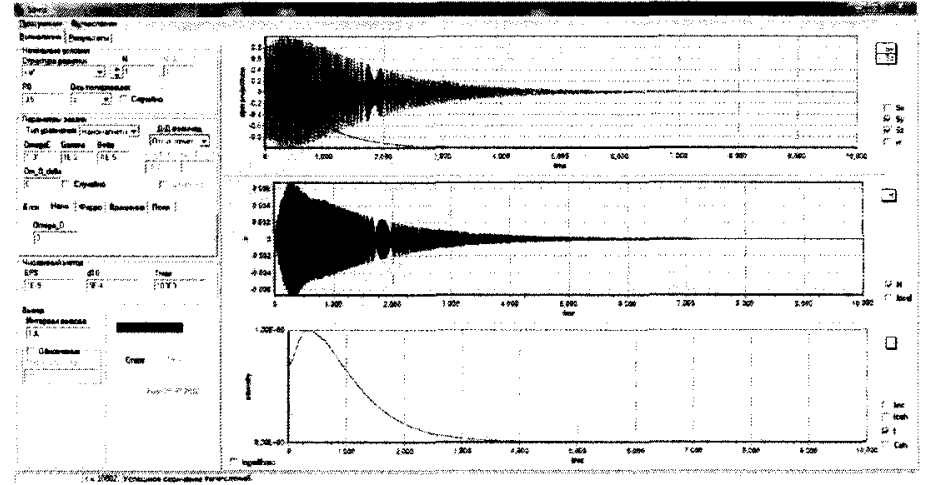

Figure 5.1.4 effect of polarization of a spin sample in case of negative $\beta=-4 \pi 10^{-5}$. Here, unlike previous cases, the system transfers to the state with maximum energy.

\section{Resonator as a Control System With Feedback}

Let us look at the system from the point of view of control theory. The resonator obviously acts a feedback system. Control theory provides a very convenient, elegant and effective framework for studying systems with feedback. In this part we will treat our system of the total spin, resonator and external magnetic field, which is applied to the spin system as a control-theory problem.

The figure below represents interaction between the spin system and the resonator as a control system.

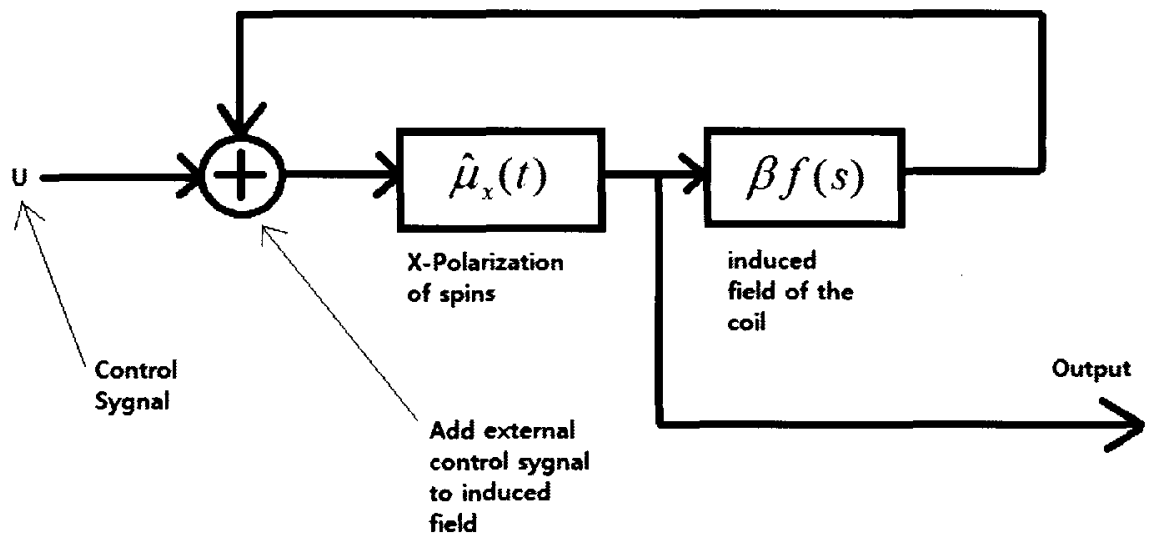

Figure 5.2.1 control-theory diagram, describing interaction between our spin system and the resonator

As we can see in this figure, the total magnetic moment of the sample affects the resonator, by inducing a magnetic field in the coil. This induced magnetic field along with the external control signal " $u$ " affects the spin sample. Magnetization of a spin sample is treated as an output. 
For this model the field of the coil will be: $\hat{H}(t)=\beta f \hat{\mu}_{x}+\hat{u}(t)$.

Thus, our control system criteria (5.1.6) can be re-written as

Fourier $\{\hat{H}(t \rightarrow \infty)\}\left(\Omega \approx \omega_{0}\right) \rightarrow 0$

Fourier $\{\hat{H}(t=\infty)\}\left(\Omega \approx \omega_{0}\right)=0$

$0=$ Fourier $\left\{\beta f \hat{\mu}_{x}+\hat{u}\right\}\left(\Omega \approx \omega_{0}\right)=$

$=$ Fourier $\left\{\beta f \hat{\mu}_{x}\right\}\left(\Omega \approx \omega_{0}\right)+$ Fourier $\{\hat{u}\}\left(\Omega \approx \omega_{0}\right)$

Fourier $\{\hat{u}(t)\}\left(\Omega \approx \omega_{0}\right) \equiv \hat{u}\left(\Omega \approx \omega_{0}\right)$.

Now, let's analyze Fourier $\left\{\beta f \hat{\mu}_{x}\right\}\left(\Omega \approx \omega_{0}\right)$ :

Fourier $\left\{\beta f \hat{\mu}_{x}\right\}(\Omega)=\frac{\beta}{\pi} \int_{0}^{\infty} e^{i \Omega t} f(s) \hat{\mu}_{x}(t) d t$.

Let's now decompose $\hat{\mu}_{x}(t)$ into a Fourier integral. Then, we'll get:

Fourier $\left\{\beta f \hat{\mu}_{x}\right\}(\Omega)=\frac{\beta}{\pi} \int_{0}^{\infty} e^{i \Omega t} f(s) \int_{-\infty}^{+\infty} \hat{\mu}_{x}\left(\Omega^{\prime}\right) e^{-i \Omega^{\prime} t} d \Omega^{\prime} d t=$

$=\frac{\beta}{\pi} \int_{0}^{\infty} \int_{-\infty}^{+\infty} e^{i \Omega t} \hat{\mu}_{x}\left(\Omega^{\prime}\right) f(s) e^{-i \Omega^{\prime} t} d \Omega^{\prime} d t=\frac{\beta}{\pi} \int_{0}^{\infty} \int_{-\infty}^{+\infty} e^{i \Omega t} \hat{\mu}_{x}\left(\Omega^{\prime}\right) e^{-i \Omega^{\prime} t} f\left(-i \Omega^{\prime}\right) d \Omega^{\prime} d t=$

$=\beta \int_{-\infty}^{+\infty} \frac{1}{\pi} \int_{0}^{+\infty} e^{i\left(\Omega-\Omega^{\prime}\right) t} d t \hat{\mu}_{x}\left(\Omega^{\prime}\right) f\left(-i \Omega^{\prime}\right) d \Omega^{\prime}$.

Since $\frac{1}{\pi} \int_{0}^{+\infty} e^{i\left(\Omega-\Omega^{\prime}\right) t} d t=\delta\left(\Omega-\Omega^{\prime}\right)$,

Fourier $\left\{\beta f \hat{\mu}_{x}\right\}\left(\Omega \approx \omega_{0}\right)=\left.\beta \int_{-\infty}^{+\infty} \delta\left(\Omega-\Omega^{\prime}\right) \hat{\mu}_{x}\left(\Omega^{\prime}\right) f\left(-i \Omega^{\prime}\right) d \Omega^{\prime}\right|_{\Omega \approx \omega_{i}}=$

$=\left.\beta f(-i \Omega) \hat{\mu}_{x}(\Omega)\right|_{\Omega \approx \omega_{1}}$

Finally,

$$
\text { Fourier }\{\hat{H}(t=\infty)\}\left(\Omega \approx \omega_{0}\right)=\left.\left(\beta f(-i \Omega) \hat{\mu}_{x}(\Omega)+\hat{u}(\Omega)\right)\right|_{\Omega \approx \omega_{1}}=0
$$

So, (5.2.1) allows us to define $\hat{\mu}_{x}$ when the system reaches equilibrium:

$$
t \rightarrow \infty: \hat{\mu}_{x}\left(\Omega \approx \omega_{0}\right)=-\left.\frac{1}{\beta f(-i \Omega)} \hat{u}(\Omega)\right|_{\Omega \approx \omega_{1}}
$$

If the control signal is very strong: 


$$
\max \left|\hat{\mu}_{x}\left(\Omega \approx \omega_{0}\right)\right|<\max \left|\frac{1}{\beta f(-i \Omega)} \hat{u}(\Omega)\right|_{\Omega \approx \omega_{u_{1}}} \mid \text {, then }\left|\hat{\mu}_{x}\left(\Omega \approx \omega_{0}\right)\right| \text { will just }
$$

reach it's maximum possible value (here, by the expression max $|x|$ we mean amplitude of changes of the value $x$ ). Considering this,

$$
t \rightarrow \infty: \hat{\mu}_{x}\left(\Omega \approx \omega_{0}\right)=T\left(\mu,-\left.\frac{1}{\beta f(-i \Omega)} \hat{u}(\Omega)\right|_{\Omega \approx \omega_{1}}\right)
$$

Where $\mu \equiv \max \left\langle\hat{\mu}_{x}\right\rangle=\max \left\langle\hat{\mu}_{y}\right\rangle=\max \left\langle\hat{\mu}_{z}\right\rangle$ - magnitude of the total magnetic moment.

$$
T(\mu, x)=\left\{\begin{array}{l}
x, \max |x|<\mu \\
\frac{\mu}{\max |x|} x, \max |x| \geq \mu
\end{array} .\right.
$$

For example, if $u(t)=h e^{i w t}$ (i.e. $u(\Omega)=h \delta(\Omega-w)$ ), then according to (5.2.3) the final state will be:

$$
\hat{\mu}_{x}\left(\Omega \approx \omega_{0}\right)=T\left(\mu,-\left.\frac{1}{\beta f(-i \Omega)} \hat{u}(\Omega-w)\right|_{\Omega \approx \omega_{0}}\right)=T\left(\mu,-\left.\frac{h}{\beta f(-i \Omega)} \delta(\Omega-w)\right|_{\Omega \approx \omega_{1}}\right)
$$

In the stationary state, when $\hat{\mu}_{z}=$ const,$\hat{\mu}_{x}(t)$ can be also represented as $\hat{\mu}_{x}(t)=e^{i\left(\omega_{0} t+\varphi\right)} \max \hat{\mu}_{x}$. (of course, we are interested only in the real parts of exponents). In this case $\hat{\mu}_{x}\left(\Omega \approx \omega_{0}\right)=e^{i \varphi} \delta\left(\Omega-\omega_{0}\right) \max \hat{\mu}_{x}$.

Thus,

$$
\begin{aligned}
& e^{i \varphi} \delta\left(\Omega-\omega_{0}\right) \max \hat{\mu}_{x}=T\left(\mu,-\left.\frac{h}{\beta f(-i \Omega)} \delta(\Omega-w)\right|_{\Omega \approx \omega_{1}}\right) . \\
& 1 . \quad \text { If } \mu>\left|\frac{h}{\beta f(-i \Omega)}\right| \text {, then } \\
& \left.e^{i \varphi} \delta\left(\Omega-\omega_{0}\right)\right|_{\Omega \approx \omega_{1}} \max \hat{\mu}_{x}=-\left.\frac{h}{\beta f(-i \Omega)} \delta(\Omega-w)\right|_{\Omega \approx \omega_{l_{1}}} \\
& e^{i \varphi} \delta(0) \max \hat{\mu}_{x}=-\frac{h}{\beta f\left(-i \omega_{0}\right)} \delta\left(\omega_{0}-w\right)
\end{aligned}
$$

Therefore, $\max \mu_{x}=-\frac{h}{\beta f\left(-i \omega_{0}\right)} e^{-i \varphi} \frac{\delta\left(\omega_{0}-w\right)}{\delta(0)}=-\frac{h}{\beta f\left(-i \omega_{0}\right)} \times\left\{\begin{array}{l}1, \omega_{0}=w \\ 0, \omega_{0} \neq w\end{array}\right.$. Thus, if $w=\omega_{0}$,

applying field

$$
\max \mu_{x}=\left|\max \mu_{x}\right|=\left|\frac{h}{\beta f\left(-i \omega_{0}\right)}\right| \text {. Thus, we can set required } \max \mu_{x} \text {, by }
$$

$$
h=\beta\left|f\left(-i \omega_{0}\right)\right| \max \mu_{x} \text {. }
$$


2. If $\mu \leq\left|\frac{h}{\beta f(-i \Omega)}\right|$, then finally, $\max \mu_{x}=\mu ; \max \mu_{z}=0$.

Let us try to solve a simple control problem: we will try to bring the system to the state with X-polarization (let's say ...) 0.223021 , provided that all the parameters are like in previous examples. So, the amplitude of controlling field should be

$$
\begin{aligned}
& h=\beta\left|f\left(-i \omega_{0}\right)\right| \max \mu_{x}: \\
& \quad h=4 \pi \times 10^{-5}\left|f\left(-i \omega_{0}\right)\right| \times 0.2=0.2 \times 10^{-5} \times 4 \pi\left|\frac{-\omega_{0}^{2}}{-\omega_{0}^{2}-2 i \gamma \omega_{0}+\omega^{2}}\right|= \\
& =0.2 \times 10^{-5} \times 4 \pi \frac{\omega_{0}^{2}}{\left|\left(\omega^{2}-\omega_{0}^{2}\right)-2 i \gamma \omega_{0}\right|}= \\
& =0.2 \times 10^{-5} \times 4 \pi \frac{\omega_{0}^{2}}{\sqrt{\left(\omega^{2}-\omega_{0}^{2}\right)^{2}+4 \gamma^{2} \omega^{2}}}= \\
& =0.2 \times 10^{-5} \times 4 \pi \frac{1^{2}}{\sqrt{(2 \times 1 \times(0.01))^{2}+4 \times 0.01^{2} \times 1.01^{2}}}= \\
& =0.00125 .
\end{aligned}
$$

As a result of simulation we get:

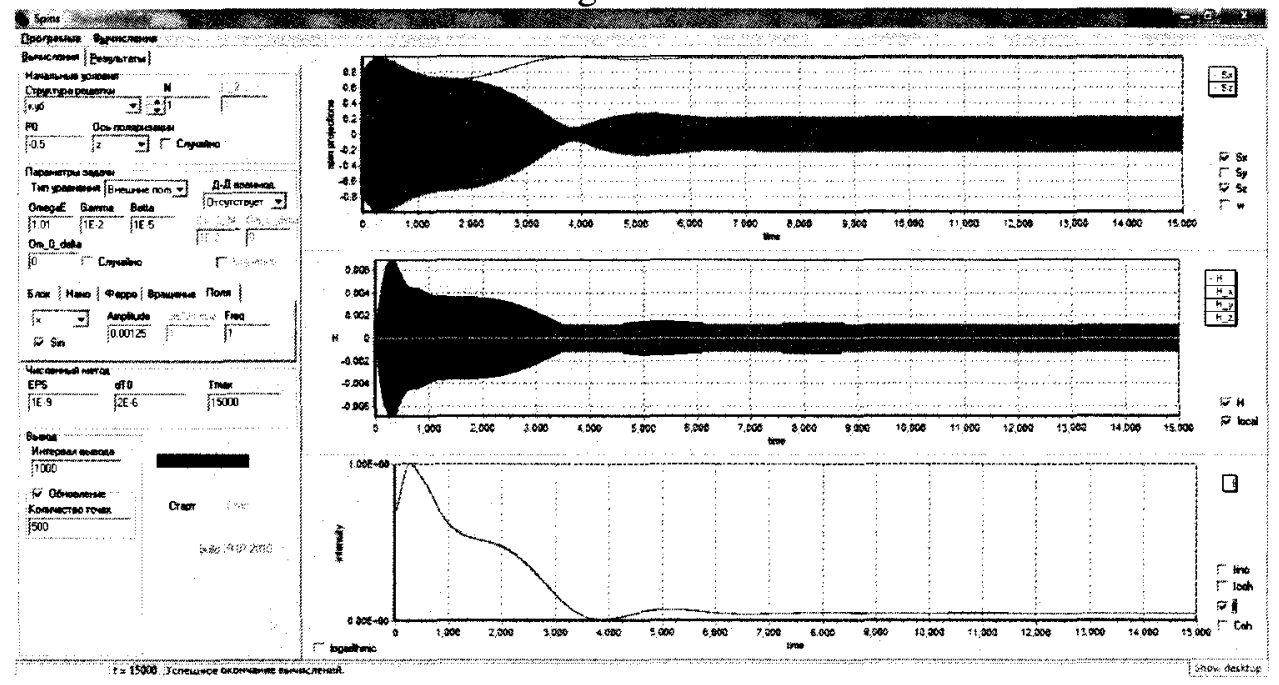

Figure 5.2.2 control of amplitude of X-component of the spin.

Here we can see that the $\mathrm{X}$-amplitude of the total magnetic moment reaches exactly the expected value (upper chart). As one can see, eventually the induced field of the resonator (second chart, dark color) reaches the same amplitude, as the external field (lighter color). 


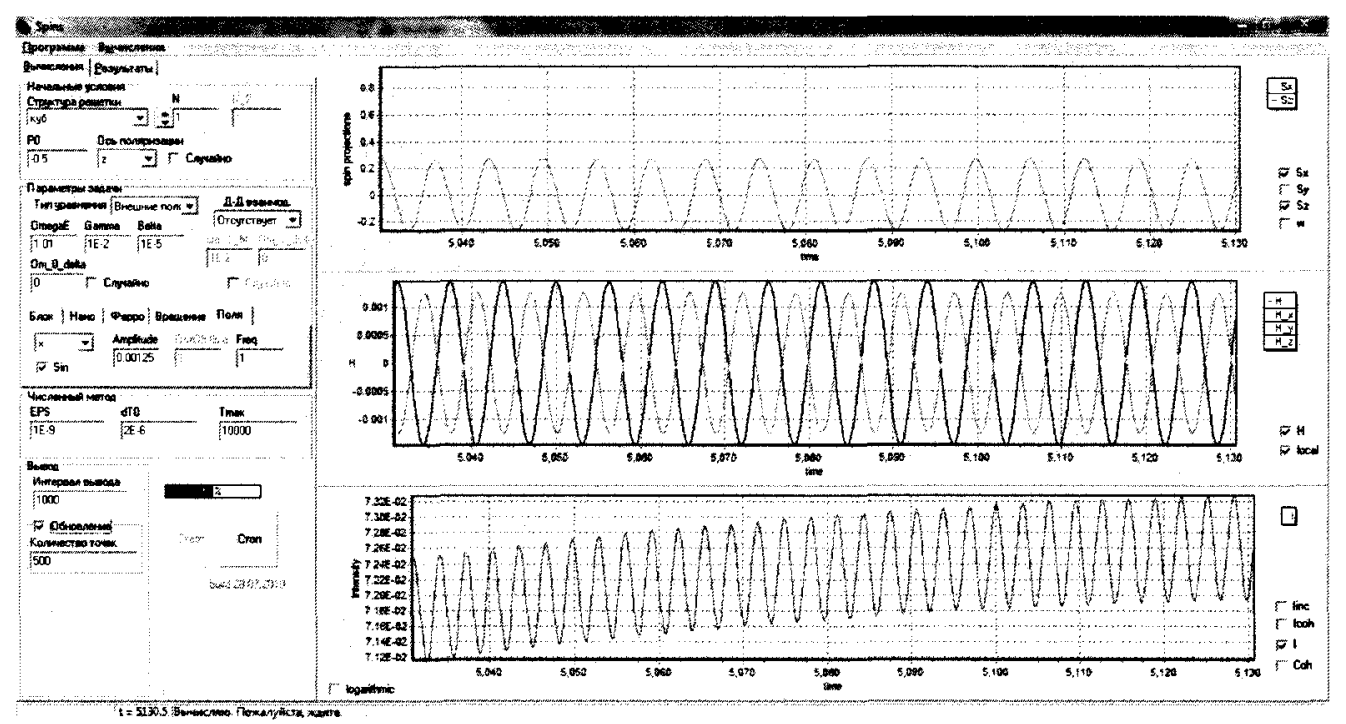

Figure 5.2.3 screenshot with the magnetic field chart, zoomed time wise.

As one can see, the induced magnetic field amplitude is greater then the control signal.

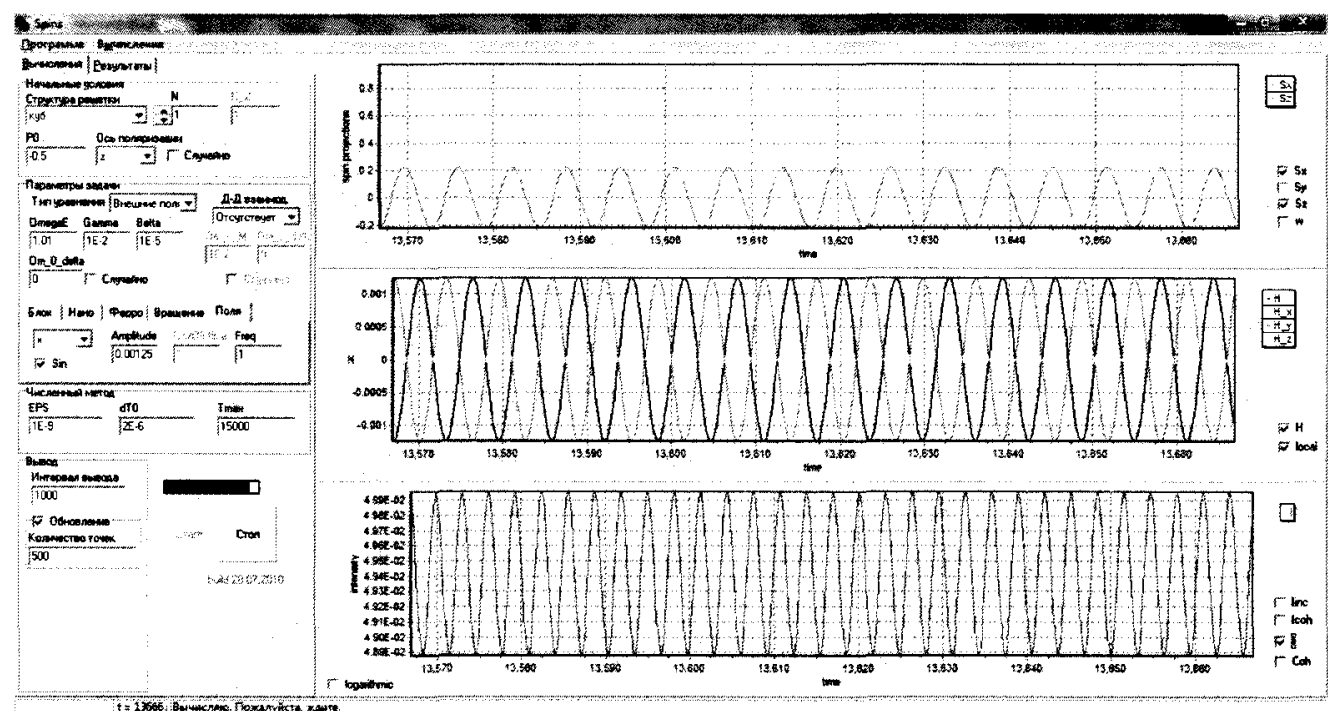

Figure 5.2.4 screenshot with the magnetic field chart, zoomed time wise (at a later time moment).

On these two figures (above) (fig. 5.2.3; 5.2.4) we can see (at different moments of time) that the induced field of the coil and the external field compensate each exactly other and their sum ("H" and "local") is eventually zero.

On the following figure one can see the similar situation as before, but with a stronger control signal, which leads to a bigger amplitude of X-oscillations of the spin. 


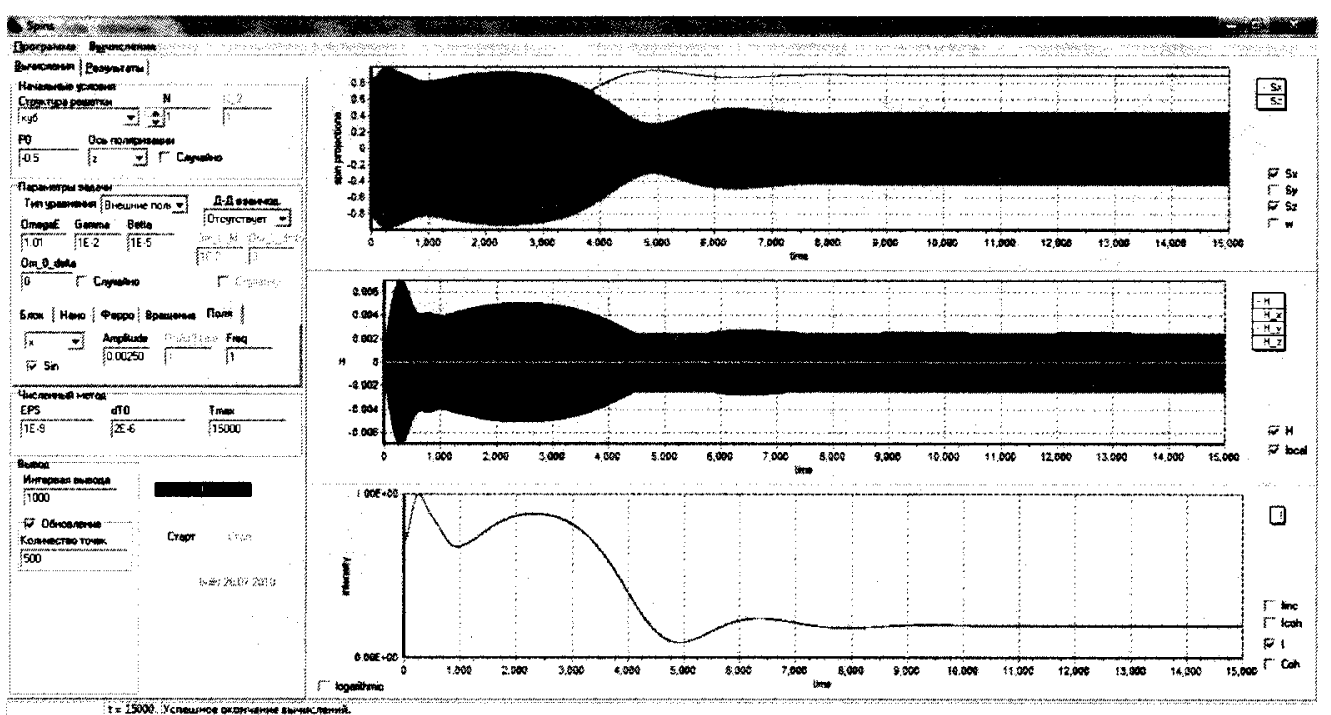

Figure 5.2.5 control of amplitude of X-component of the spin (bigger control signal).

Here (fig. 5.2.5) the external field is twice as large (0.00250), so the $\mathrm{X}$ polarization amplitude reaches 0.446096 .

The next figure shows a situation, when $\mu \leq\left|\frac{h}{\beta f(-i \Omega)}\right|$

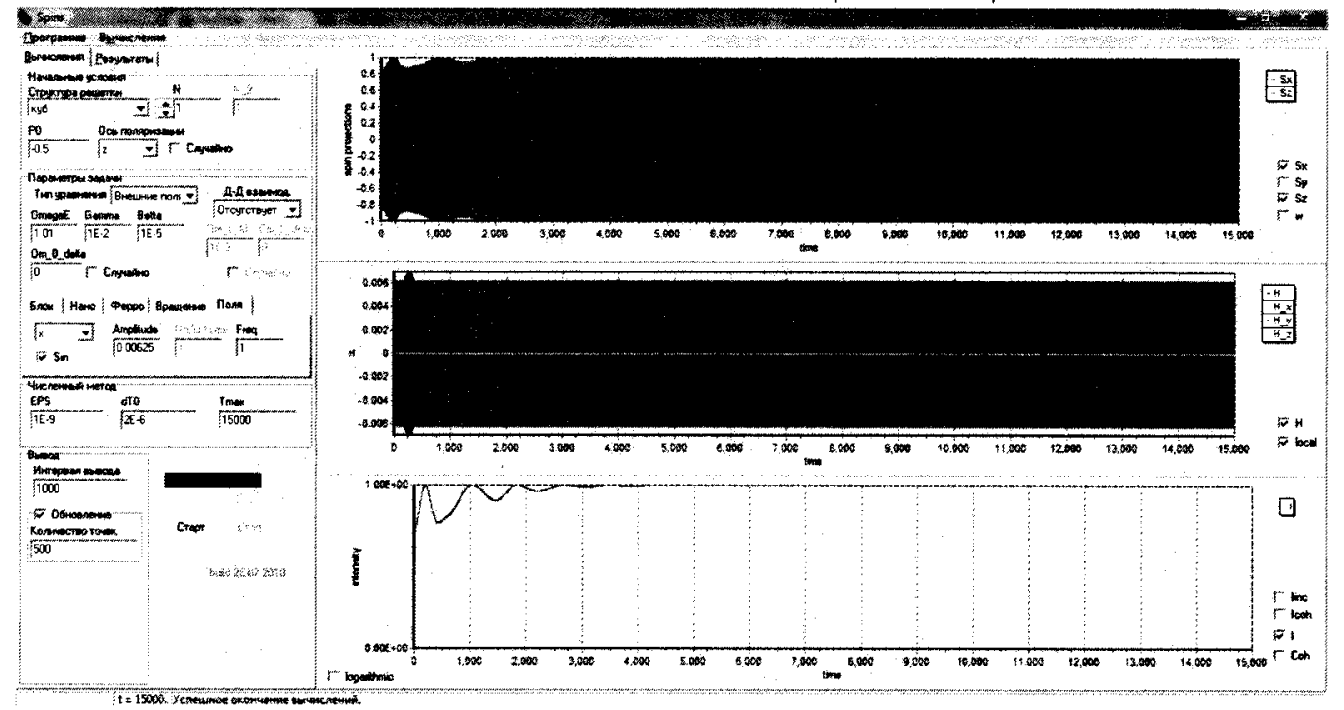

Figure 5.2.6 control of amplitude of X-component of the spin (very big control signal).

Here the external field is 5 times greater than in the first case $(0.00625)$, so the normalized X-polarization amplitude reaches 0.999206 . The normalized Zpolarization goes down 0.00383063 . This is an illustration of the case, when $\mu \leq\left|\frac{h}{\beta f(-i \Omega)}\right|$. 


\section{Conclusion:}

We have shown that properly adjusted coil and external field can control the polarization of a spin sample instead of simple flips (Rabi oscillations).

Those requirements are:

$\mu \beta f \hat{\mu}_{x}(t) \sqsubset \gamma-\gamma$ should be big enough,

$\mu \square\left|\frac{h}{\beta f\left(-i \omega_{0}\right)}\right|-h$ shouldn't be too big.

So, using the idea described above we can control polarization of a spin sample.

\section{Generalization of the Control Theory Approach}

Let us now generalize the idea used in parts V.1 and V.2. The approach, used in those parts led us to obtain rather precise results. Potentially, this approach can be applied not only to the spin system and the resonator, but to any two interacting systems.

In part V.1. we were studying the situation without an external control signal, but taking into account GSHE of the resonator. In part V. 2 we considered a system with the external control signal, but without GSHE (because it disappears with time). We can easily combine and generalize these two situations by formally treating GSHE as an external control signal.

Consider two systems (let's call them "q" and "e"). The Hamiltonian of this system will be $\hat{H}=\hat{H}(q, e)$. (symbols $q$ and $\mathrm{e}$ in the formulas mean sets of generalized momentums and coordinates of $\mathrm{q}$ and e-systems respectively).

The idea is to treat the problem as a control problem with feedback. In this case $q$ will be our controlled system and $\mathrm{e}$ - will be the feedback system, just like in the figure below.

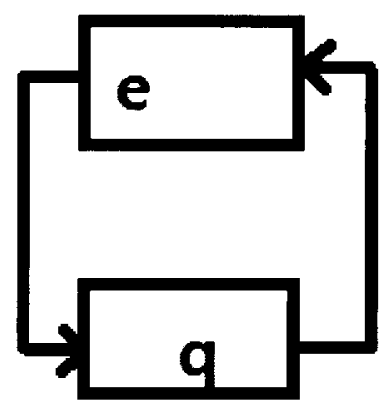

Figure 5.3.1 general control-theory diagram between two subsystems 
We will treat symbols $e$ and $q$ as a set of generalized coordinates of "q" and " $\mathrm{e}$ " respectively. The general idea is the following:

1. Get transfer function and the control signal of "e"

In order to do this, we have to:

1.1) Write down Hamilton's equation for " $\mathrm{e}$ ", treating “q" as a set of input signals.

1.2) Bring the system of Hamilton's equations to the form of differential equations of higher order.

1.3) Formally solve these equations: after this, we will get a combination of Particular Solution of the Equation (PSE) and General Solution of Equation (GSE)

1.3.1) PSE can be represented in terms of transfer function: $e=G_{e}(s, q)$, where $s=\frac{\partial}{\partial t}$.

1.3.2) GSE depicts behavior of system "e" without influence of "q" (we will write GSE as $\hat{u}_{e}(t)$ ).

2. Plug "e" (now in terms of $G_{e}(s, q)$ and $\hat{u}_{e}(t)$ ) in the Hamiltonian. Thus, from the Hamiltonian in terms of $\hat{H}=\hat{H}(q, e)$ we switch to $\hat{H}=\hat{H}\left(q, G_{e}(s, q), \hat{u}_{e}(t)\right)$. Thus, we formally exclude "e" from the Hamiltonian and finally arrive at the problem for one system "q". Thus, the diagram (fig. 5.3.1) can be re-drawn in the following way:

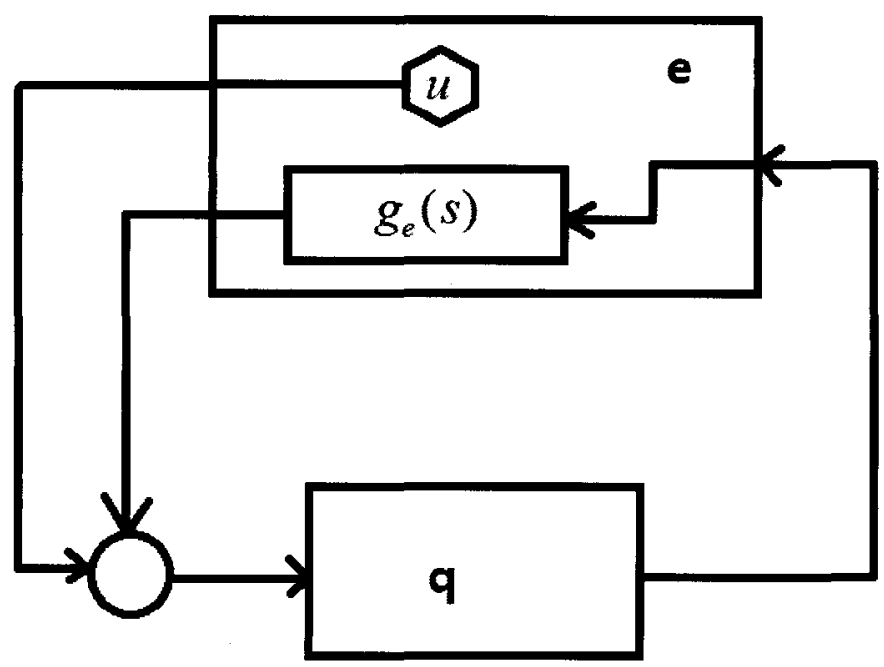

Figure 5.3.2 detailed control diagram of interaction between two systems.

In this figure one can see that system "e" acts on system "q" in two ways: as a transfer function $g_{e}(s)$, which depicts the reaction of " $\mathrm{e}$ " on the output of "q"; and as a generator of a control signal for "q". Signal $u$ is nothing but a general solution of differential equations for " $e$ " in the absence of " $q$ ". 
And finally, to get rid of the "traces" of "e", we can re-draw this diagram so that it looks exactly as a standard model, which can be treated by means of control theory:

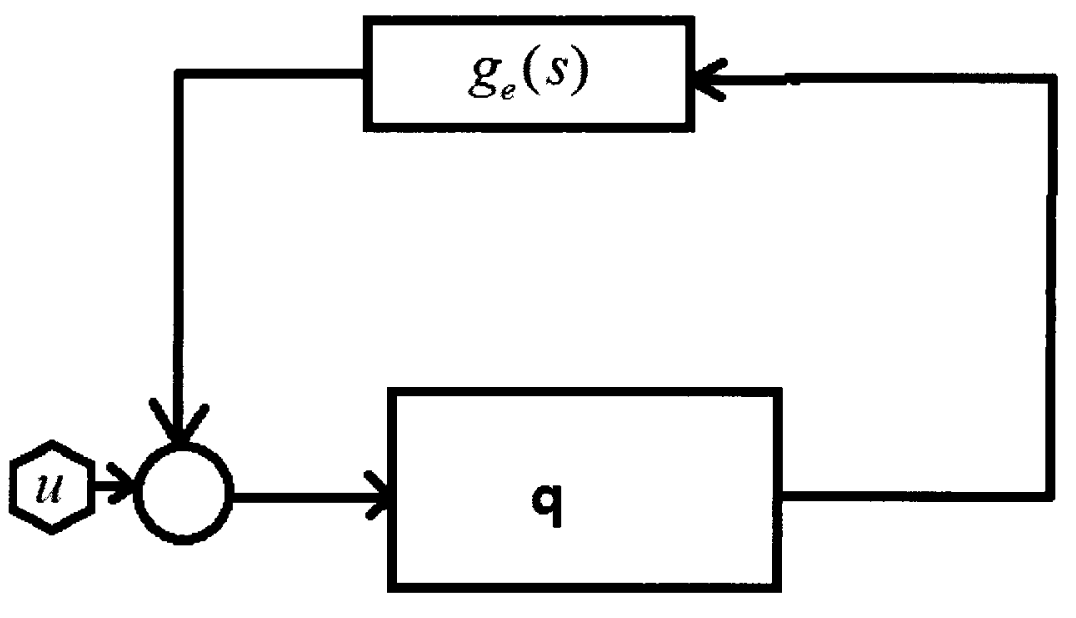

Figure 5.3.3 re-arranged detailed control diagram of interaction between two systems.

Finally, the problem of interaction between the two subsystems can be formally brought to the standard control problem, which can be solved by welldeveloped methods of control theory. 


\section{CONCLUSION}

In parts I and II we were able to see that for some important observables (such as spin components) and many forms of the Hamiltonian, classical and quantum equations give us identical results even for microscopic objects. Even such quantum effects as a difference between the strength of interactions due to secular and nonsecular parts of the Hamiltonian can be obtained in a classical model due to the correspondence principle. In addition, the Heisenberg approach combined with the proven correspondence principle allows us to model the evolution of many different observables instead of just energy levels, which quantum energy provides. However, we were able to see that the correspondence principle is valid not for all the observables and not for all kinds of Hamiltonians. Thus, the approach, based on the combination of the Heisenberg equations with the correspondence principle can give us correct results for some observables, while producing discrepancies (which vanish in the classical limit, though) for other observables. The question, which arises from this fact is: is it possible to choose some other set of generalized coordinates (instead of spin operators $\left\{\hat{S}_{j}^{\alpha}\right\}$ ), such that we can obtain other correspondence principles? If such a possibility exists, we can obtain correct results for a broader "spectrum" of observables, if we use a different set of generalized coordinates. In future we are planning to investigate such a possibility.

In part III we presented the mathematical model which is used for simulation of processes, which occur in the spin system in the presence of feedback via a resonator. We had set up a system of differential equations for spins and for the resonator, which provides the feedback magnetic field.

In part IV we switched to the rotating frame, which allows us to get rid of big Zeeman terms and frequent oscillations in differential equations. A simple procedure for classical magnetic resonance theory, switching to the rotating frame becomes more difficult due to the resonator. As a result, we were only able to get rid of big terms, but not frequently-oscillating terms. Finally, an algorithm, which works in both (stationary and rotating) frames has been elaborated.

After setting up all the basic principles and mathematical models, in part $\mathrm{V}$ we investigated the behavior of a spin system in presence of feedback via a resonator. It turns out that a combination of control theory with the Heisenberg approach to apply for quantum theory is rather fruitful. Using this combined approach, we have arrived to some non-trivial theoretical results, which are supported by simulation. In the future we are planning to find some applications of described effects. A potential advantage of using a resonator for feedback is its sensitivity to horizontal projections of magnetization, while classical magnetic resonance deals only with energy. 


\section{REFERENCES}

[1] Slichter, C. P. (1963) Principles of magnetic resonance, with examples from solid state physics, New York, Harper \& Row

[2] Abragam, A., Goldman, M. (1982) Nuclear magnetism : order and disorder, Oxford, Clarendon Press

[3] Henner, V. K., Yukalov, V. I., Kharebov, P. V., Yukalova, E. P. (2008) Collective spin dynamics in magnetic nanomaterials, The International Conference on Theoretical Physics 'Dubna-Nano2008',Journal of Physics: Conference Series 129 [4] Yukalov, V., I. (2002)Superradiant Operation of Spin Maser, Laser Physics, Vol. 12 , No. 8 


\title{
CURRICULUM VITAE
}

\author{
Andrey R. Klots \\ Natural Science Building, \\ University of Louisville \\ Louisville, KY 40292 \\ Telephone : 1(502)2962426 \\ e-mail : klotsandrey@gmail.com \\ EDUCATION: University of Louisville, \\ Louisville, KY \\ Candidate for M.S. degree, Department of \\ $08 / 2009-$ \\ Physics and Astronomy. \\ $05 / 2011$ \\ (expected) \\ Emphasis: Nuclear Magnetic Resonance and \\ Spin dynamics \\ Perm State University, \\ Perm, Russia \\ B.A. degree (with honors), Physics \\ $09 / 2004$ \\ Department. \\ 07/2009 \\ Thesis: "Building an automatic control system \\ for optimization of parameters of \\ optoelectronic components for telemetry \\ systems" \\ Advisor: Dr. D. I. Shevtsov \\ WORK Engineering technologist at \\ EXPERIENCE Science \& Technology Center at the Perm \\ I \\ Scientific Industrial Instrument-Making \\ Perm, Russia \\ EMPLOYME Company \\ $11 / 2008-$ \\ $07 / 2009$ \\ NT \\ Building an automatic system for controlling \\ vital parameters of an optic fiber during \\ temperature tests. \\ Requires professional skills (in computer \\ science, electronics and optics) and a high \\ degree of self-reliance. \\ Designing a working model of a telemetry \\ system for monitoring biometric parameters \\ of a pilot using fiber-optic sensors. \\ Requires skills in computer science,
}


electronics (especially, DSP-processors and electrical measurement techniques), optics and basic knowledge biophysics (cardiography, encephalography, etc.). Also requires ability to work in team.

RESEARCH Currently working with Dr. V. K. Henner, professor of physics at the University of Louisville and Perm State University. We are studying influence of a resonator on interactions between spins in magnetic resonance. We are also developing a theoretical framework which allows us to perform computer modeling of magnetic resonance processes.

As an undergraduate, was studying spin dynamics under supervision of Dr. A. Oshepkov (Perm State University).

We were studying dynamics of spin $1 / 2$ (as a qbit) (from the point of view of control theory) and performing modeling in MATLAB-Simulink environment.

TEACHING Teaching assistant at the University of Louisville

Teaching lab courses in "Electricity and Magnetism" and "Mechanics" - courses.

Grading physics tests and homework

PUBLICATIO "Modelling of Qbit Dynamics in MATLABNS Simulink environment", Perm State University

AWARDS and HONORS

2 championship

$2^{\text {nd }}$ place on the department's physics championship

SKILLS and QUALIFICAT IONS
Computer: MATLAB (+Simulink), Mathcad

Programming: $\mathrm{C} / \mathrm{C}++$; $\mathrm{C}++$ Builder; PHP, mySQL, HTML, Assembler; Pascal; Delphi; Basic;

Electronics: DSP-Processors, Building electric circuits, $\mathrm{P}-\mathrm{Cad}$

Language skills: Russian (native speaker), German (basic level)
Louisville, KY;

Perm, Russia

09/2008 - present

Perm, Russia

$09 / 2007-$

$06 / 2008$

09/2009 - present

Perm, Russia, 2007

Perm, Russia, 2007

Perm, Russia, 2006 
Personal Qualities: creative, enthusiastic, hardworking, responsible, confident. 